\title{
Koszul-Tate resolutions as cofibrant replacements of algebras over differential operators
}

\author{
Gennaro di Brino, Damjan Pištalo, and Norbert Poncin*
}

\begin{abstract}
Homotopical geometry over differential operators is a convenient setting for a coordinatefree investigation of nonlinear partial differential equations modulo symmetries. One of the first issues one meets in the functor of points approach to homotopical $\mathcal{D}$-geometry, is the question of a model structure on the category $\operatorname{DGAlg}(\mathcal{D})$ of differential non-negatively graded $\mathcal{O}$-quasi-coherent sheaves of commutative algebras over the sheaf $\mathcal{D}$ of differential operators of an appropriate underlying variety $(X, \mathcal{O})$. We define a cofibrantly generated model structure on $\operatorname{DGAlg}(\mathcal{D})$ via the definition of its weak equivalences and its fibrations, characterize the class of cofibrations, and build an explicit functorial 'cofibration trivial fibration' factorization. We then use the latter to get a functorial model categorical Koszul-Tate resolution for $\mathcal{D}$-algebraic 'on-shell function' algebras (which contains the classical Koszul-Tate resolution). The paper is also the starting point for a homotopical $\mathcal{D}$-geometric Batalin-Vilkovisky formalism.
\end{abstract}

MSC 2010: 18G55, 16E45, 35A27, 32C38, 16S32, 18G10

Keywords: Differential operator, $\mathcal{D}$-module, model category, relative Sullivan $\mathcal{D}$-algebra, homotopical geometry, $\mathcal{D}$-geometry, functor of points, Koszul-Tate resolution, Batalin-Vilkovisky formalism

\section{Contents}

1 Introduction

2 Conventions and notation

3 Sheaves of modules

4 D-modules and $\mathcal{D}$-algebras

4.1 Construction of $\mathcal{D}$-modules from $\mathcal{O}$-modules . . . . . . . . . . . . . . . . 6

4.2 Closed symmetric monoidal structure on $\operatorname{Mod}(\mathcal{D}) \ldots \ldots \ldots$. . . . . . . 6

4.3 Commutative $\mathcal{D}$-algebras . . . . . . . . . . . . . . . . . G

${ }^{*}$ University of Luxembourg, Mathematics Research Unit, 4364 Esch-sur-Alzette, Luxembourg, gennaro.dibrino@gmail.com, damjan.pistalo@uni.lu,norbert.poncin@uni.lu 
5 Differential graded $\mathcal{D}$-modules and differential graded $\mathcal{D}$-algebras

5.1 Monoidal categorical equivalence between chain complexes of $\mathcal{D}_{X}$-modules and their global sections

5.2 Differential graded $\mathcal{D}_{X}$-algebras vs. differential graded $\mathcal{D}_{X}(X)$-algebras . . . . 9 9

5.3 The category DGDA . . . . . . . . . . . . . . . . . . . . 10

6 Finitely generated model structure on DGDM 11

7 Finitely generated model structure on DGDA 13

7.1 Adjoint functors between DGDM and DGDA . . . . . . . . . . . . . 13

7.2 Relative Sullivan $\mathcal{D}$-algebras . . . . . . . . . . . . . . . . 14

7.3 Quillen's transfer theorem . . . . . . . . . . . . . . . . . . 15

7.4 Proof of Condition 1 of Theorem $18 \ldots \ldots \ldots \ldots \ldots$

7.5 Proof of Condition 2 of Theorem $18 \ldots \ldots \ldots \ldots \ldots \ldots$

7.6 Transferred model structure . . . . . . . . . . . . . . . . . . . . 21

8 Description of DGDA-cofibrations 22

8.1 Preliminaries . . . . . . . . . . . . . . . . . . . . 22

8.2 DGDA-cofibrations . . . . . . . . . . . . . . . . . . . 26

9 Explicit functorial factorizations 31

10 First remarks on Koszul-Tate resolutions

10.1 Undercategories of model categories . . . . . . . . . . . . . . . . 38

10.2 Basics of jet bundle formalism . . . . . . . . . . . . . . 38

10.3 Revisiting classical Koszul-Tate resolution . . . . . . . . . . . . . . . . 39

$10.4 \mathcal{D}$-algebraic version of the Koszul-Tate resolution . . . . . . . . . . . . 41

11 Appendices

11.1 Appendix 1 - Quasi-coherent sheaves of modules . . . . . . . . . . . . 42

11.2 Appendix 2 - D-modules . . . . . . . . . . . . . . . 43

11.3 Appendix 3 -Sheaves versus global sections . . . . . . . . . . . . 44

11.4 Appendix 4 - Model categories . . . . . . . . . . . . . . . . . . . . 44

11.5 Appendix 5 - Invariants versus coinvariants . . . . . . . . . . . . 46

11.6 Appendix 6 - Proof of Theorem $29 \ldots \ldots \ldots \ldots \ldots \ldots$

11.7 Appendix 7 - Explicit functorial cofibrant replacement functor . . . . . . . 48

12 Acknowledgments 51

\section{Introduction}

The solution functor of a linear $\operatorname{PDE} D \cdot m=0$ is a functor $\operatorname{Sol}: \operatorname{Mod}(\mathcal{D}) \rightarrow \operatorname{Set}$ defined on the category of left modules over the ring $\mathcal{D}$ of linear differential operators of a suitable 
underlying space: for $D \in \mathcal{D}$ and $M \in \operatorname{Mod}(\mathcal{D})$, we have

$$
\operatorname{Sol}(M)=\{m \in M: D \cdot m=0\} .
$$

For a polynomial PDE, we get a representable functor $\operatorname{Sol}: \operatorname{Alg}(\mathcal{D}) \rightarrow$ Set defined on the category of $\mathcal{D}$-algebras, i.e., of commutative monoids in $\operatorname{Mod}(\mathcal{D})$. According to [BD04, the solution functor of a nonlinear PDE should be viewed as a 'locally representable' sheaf Sol : $\operatorname{Alg}(\mathcal{D}) \rightarrow$ Set. To allow for still more general spaces, sheaves $\operatorname{Alg}(\mathcal{D}) \rightarrow$ SSet valued in simplicial sets, or sheaves $\operatorname{DGAlg}(\mathcal{D}) \rightarrow$ SSet on (the opposite of ) the category $\operatorname{DGAlg}(\mathcal{D})$ of differential graded $\mathcal{D}$-algebras, have to be considered.

More precisely, when constructing a derived algebraic variant of the jet bundle approach to the Lagrangian Batalin-Vilkovisky formalism, not, as usual, in the world of function algebras, but, dually, on the space side, we first consider the quotient of the infinite jet space by the global gauge symmetries. It turns out [BPP17] that this quotient should be thought of as a 1-geometric derived $X$ - $\mathcal{D}_{X}$-stack, where $X$ is an underlying smooth affine algebraic variety. This new homotopical algebraic $\mathcal{D}$-geometry provides in particular a convenient way to encode total derivatives and it allows actually to recover the classical Batalin-Vilkovisky complex as a specific case of its general constructions [PP17b]. In the functor of points approach to spaces, the derived $X$-D $\mathcal{D}_{X}$-stacks $F$ are those presheaves $F: \operatorname{DGAlg}(\mathcal{D}) \rightarrow \operatorname{SSet}$ that satisfy the fibrant object (sheaf-)condition for the local model structure on the presheaf category $\operatorname{Fun}(\operatorname{DGAlg}(\mathcal{D})$, SSet $)$ - the category of derived $X$ - $\mathcal{D}_{X}$-stacks is in fact the homotopy category of this model category of functors. More precisely, the choice of an adequate model (pre-)topology allows us to construct the local model structure, via a double Bousfield localization, from the global model structure of the considered presheaf category, which is implemented 'object-wise' by the model structure of the target category SSet. The first of the two Bousfield localizations is the localization of this global model structure with respect to the weak equivalences of the (category opposite to the) source category $\operatorname{DGAlg}(\mathcal{D})$. Furthermore, the $\mathcal{D}$-geometric counterpart of an algebra $C^{\infty}(\Sigma)$ of on-shell functions is an algebra $A \in \operatorname{Alg}(\mathcal{D}) \subset \operatorname{DGAlg}(\mathcal{D})$, and it appears [PP17a that the Koszul-Tate resolution of $C^{\infty}(\Sigma)$ corresponds to the cofibrant replacement of $A$ in a coslice category of $\operatorname{DGAlg}(\mathcal{D})$.

In view of the two preceding reasons, it is clear that our first task is the definition of a model structure on $\operatorname{DGAlg}(\mathcal{D})$ (we draw the attention of the reader to the fact that we will use two different definitions of model categories, namely the definition of [GS06] and that of [Hov07] - for the details we refer to Appendix 11.4). In the present paper, we give an explicit description of a cofibrantly generated model structure on the category $\operatorname{DGAlg}(\mathcal{D})$ of differential non-negatively graded $\mathcal{O}$-quasi-coherent sheaves of commutative algebras over the sheaf $\mathcal{D}$ of differential operators of a smooth affine algebraic variety $(X, \mathcal{O})$. In particular, we characterize the cofibrations as the retracts of the relative Sullivan $\mathcal{D}$-algebras and we give an explicit functorial 'Cof - TrivFib' factorization (as well as the corresponding functorial cofibrant replacement functor - which is specific to our setting and is of course different from the one provided, for arbitrary cofibrantly generated model categories, by the small object argument). 
To develop the afore-mentioned homotopical $\mathcal{D}$-geometry, we have to show inter alia that the triplet $(\operatorname{DGMod}(\mathcal{D}), \operatorname{DGMod}(\mathcal{D}), \operatorname{DGAlg}(\mathcal{D}))$ is a homotopical algebraic context [TV08. This includes proving that the model category $\operatorname{DGAlg}(\mathcal{D})$ is proper and that the base change functor $\mathcal{B} \otimes_{\mathcal{A}}-$, from modules in $\operatorname{DGMod}(\mathcal{D})$ over $\mathcal{A} \in \operatorname{DGAlg}(\mathcal{D})$ to modules over $\mathcal{B} \in \mathcal{A} \downarrow \operatorname{DGAlg}(\mathcal{D})$, preserves weak equivalences. These results [BPP17] are based on our characterization of cofibrations in DGAlg $(\mathcal{D})$, as well as on the explicit functorial 'Cof - TrivFib' factorization.

Notice finally that our two assumptions - smooth and affine - on the underlying variety $X$ are necessary. Exactly the same smoothness condition is indeed used in [BD04] [Remark p.56], since for an arbitrary singular scheme $X$, the notion of left $\mathcal{D}_{X}$-module is meaningless. On the other hand, the assumption that $X$ is affine is needed to substitute global sections of sheaves to the sheaves themselves, i.e., to replace the category of differential non-negatively graded $\mathcal{O}$-quasi-coherent sheaves of commutative algebras over the sheaf $\mathcal{D}$ by the category of differential non-negatively graded commutative algebras over the ring $\mathcal{D}(X)$ of global sections of $\mathcal{D}$. However, this confinement is not merely a comfort solution: the existence of the projective model structure - that we transfer from $\operatorname{DGMod}(\mathcal{D})$ to $\operatorname{DGAlg}(\mathcal{D})$ - requires that the underlying category has enough projectives, and this is in general not the case for a category of sheaves over a not necessarily affine scheme [Gil06], Har97, Ex.III.6.2]. In addition, the confinement to the affine case allows us to use the artefacts of the model categorical environment, as well as to extract the fundamental structure of the main actors of the considered problem, which may then be extended to an arbitrary smooth scheme $X$ [PP17a].

Let us still stress that the special behavior of the noncommutative ring $\mathcal{D}$ turns out to be a source of possibilities, as well as a source of problems. For instance, a differential graded commutative algebra over a field or a commutative ring $k$ is a commutative monoid in the category of differential graded $k$-modules. The extension of this concept to noncommutative rings $R$ is problematic, since the category of differential graded (left) $R$-modules is not symmetric monoidal. In the case $R=\mathcal{D}$, we deal with differential graded (left) $\mathcal{D}$-modules and these are symmetric monoidal - and also closed. However, the tensor product and the internal Hom are taken, not over $\mathcal{D}$, but over $\mathcal{O}$ : one considers the $\mathcal{O}$-modules given, for $M, N \in \operatorname{DGMod}(\mathcal{D})$, by $M \otimes_{\mathcal{O}} N$ and $\operatorname{Hom}_{\mathcal{O}}(M, N)$, and shows that their $\mathcal{O}$-module structures can be extended to $\mathcal{D}$-module structures [HTT08]. This and other - in particular related - specificities must be kept in mind throughout the whole paper.

\section{Conventions and notation}

According to the anglo-saxon nomenclature, we consider the number 0 as being neither positive, nor negative.

All the rings used in this text are implicitly assumed to be unital. 


\section{Sheaves of modules}

Let Top be the category of topological spaces and, for $X \in$ Top, let Open $_{X}$ be the category of open subsets of $X$. If $\mathcal{R}_{X}$ is a sheaf of rings, a left $\mathcal{R}_{X}$-module is a sheaf $\mathcal{P}_{X}$, such that, for each $U \in \operatorname{Open}_{X}, \mathcal{P}_{X}(U)$ is an $\mathcal{R}_{X}(U)$-module, and the $\mathcal{R}_{X}(U)$-actions are compatible with the restrictions. We denote by $\operatorname{Mod}\left(\mathcal{R}_{X}\right)$ the abelian category of $\mathcal{R}_{X}$-modules and of their (naturally defined) morphisms.

In the following, we omit subscript $X$ if no confusion arises.

If $\mathcal{P}, \mathcal{Q} \in \operatorname{Mod}(\mathcal{R})$, the (internal) Hom denoted by $\mathcal{H o m}_{\mathcal{R}}(\mathcal{P}, \mathcal{Q})$ is the sheaf of abelian groups (of $\mathcal{R}$-modules, i.e., is the element of $\operatorname{Mod}(\mathcal{R})$, if $\mathcal{R}$ is commutative) that is defined by

$$
\mathcal{H o m}_{\mathcal{R}}(\mathcal{P}, \mathcal{Q})(U):=\operatorname{Hom}_{\left.\mathcal{R}\right|_{U}}\left(\left.\mathcal{P}\right|_{U},\left.\mathcal{Q}\right|_{U}\right)
$$

$U \in \operatorname{Open}_{X}$. The RHS is made of the morphisms of (pre)sheaves of $\left.\mathcal{R}\right|_{U}$-modules, i.e., of the families $\phi_{V}: \mathcal{P}(V) \rightarrow \mathcal{Q}(V), V \in$ Open $_{U}$, of $\mathcal{R}(V)$-linear maps that commute with restrictions. Note that $\mathcal{H o m}_{\mathcal{R}}(\mathcal{P}, \mathcal{Q})$ is a sheaf of abelian groups, whereas $\operatorname{Hom}_{\mathcal{R}}(\mathcal{P}, \mathcal{Q})$ is the abelian group of morphisms of (pre)sheaves of $\mathcal{R}$-modules. We thus obtain a bi-functor

$$
\mathcal{H o m}_{\mathcal{R}}(\bullet, \bullet):(\operatorname{Mod}(\mathcal{R}))^{\text {op }} \times \operatorname{Mod}(\mathcal{R}) \rightarrow \operatorname{Sh}(X),
$$

valued in the category of sheaves of abelian groups, which is left exact in both arguments.

Further, if $\mathcal{P} \in \operatorname{Mod}\left(\mathcal{R}^{\mathrm{op}}\right)$ and $\mathcal{Q} \in \operatorname{Mod}(\mathcal{R})$, we denote by $\mathcal{P} \otimes_{\mathcal{R}} \mathcal{Q}$ the sheaf of abelian groups (of $\mathcal{R}$-modules, if $\mathcal{R}$ is commutative) associated to the presheaf

$$
\left(\mathcal{P} \oslash_{\mathcal{R}} \mathcal{Q}\right)(U):=\mathcal{P}(U) \otimes_{\mathcal{R}(U)} \mathcal{Q}(U)
$$

$U \in \operatorname{Open}_{X}$. The bi-functor

$$
\text { - } \otimes_{\mathcal{R}} \bullet: \operatorname{Mod}\left(\mathcal{R}^{\mathrm{op}}\right) \times \operatorname{Mod}(\mathcal{R}) \rightarrow \operatorname{Sh}(X)
$$

is right exact in its two arguments.

If $\mathcal{S}$ is a sheaf of commutative rings and $\mathcal{R}$ a sheaf of rings, and if $\mathcal{S} \rightarrow \mathcal{R}$ is a morphism of sheafs of rings, whose image is contained in the center of $\mathcal{R}$, we say that $\mathcal{R}$ is a sheaf of $\mathcal{S}$-algebras. Remark that, in this case, the above functors $\mathcal{H o m}_{\mathcal{R}}(\bullet, \bullet)$ and $\bullet \otimes_{\mathcal{R}} \bullet$ are valued in $\operatorname{Mod}(\mathcal{S})$.

\section{D-modules and $\mathcal{D}$-algebras}

Depending on the author(s), the concept of $\mathcal{D}$-module is considered over a base space $X$ that is a finite-dimensional smooth [Cos11] or complex [KS90] manifold, or a smooth algebraic variety [HTT08] or scheme BD04, over a fixed base field $\mathbb{K}$ of characteristic zero. We denote by $\mathcal{O}_{X}$ (resp., $\Theta_{X}, \mathcal{D}_{X}$ ) the sheaf of functions (resp., vector fields, differential operators acting 
on functions) of $X$, and take an interest in the category $\operatorname{Mod}\left(\mathcal{O}_{X}\right)\left(\operatorname{resp} ., \operatorname{Mod}\left(\mathcal{D}_{X}\right)\right)$ of $\mathcal{O}_{X^{-}}$ modules (resp., $\mathcal{D}_{X}$-modules).

Sometimes a (sheaf of) $\mathcal{D}_{X}$-module(s) is systematically required to be coherent or quasicoherent as (sheaf of) $\mathcal{O}_{X}$-module(s). In this text, we will explicitly mention such extra assumptions.

\subsection{Construction of $\mathcal{D}$-modules from $\mathcal{O}$-modules}

It is worth recalling the following

Proposition 1. Let $\mathcal{M}_{X}$ be an $\mathcal{O}_{X}$-module. A left $\mathcal{D}_{X}$-module structure on $\mathcal{M}_{X}$ that extends its $\mathcal{O}_{X}$-module structure is equivalent to a $\mathbb{K}$-linear morphism

$$
\nabla: \Theta_{X} \rightarrow \mathcal{E} n d_{\mathbb{K}}\left(\mathcal{M}_{X}\right)
$$

such that, for all $f \in \mathcal{O}_{X}, \theta, \theta^{\prime} \in \Theta_{X}$, and all $m \in \mathcal{M}_{X}$,

1. $\nabla_{f \theta} m=f \cdot \nabla_{\theta} m$,

2. $\nabla_{\theta}(f \cdot m)=f \cdot \nabla_{\theta} m+\theta(f) \cdot m$,

3. $\nabla_{\left[\theta, \theta^{\prime}\right]} m=\left[\nabla_{\theta}, \nabla_{\theta^{\prime}}\right] m$.

In the following, we omit again subscript $X$, whenever possible.

In Proposition 11, the target $\mathcal{E} n d_{\mathbb{K}}(\mathcal{M})$ is interpreted in the sense of Equation (11), and $\nabla$ is viewed as a morphism of sheaves of $\mathbb{K}$-vector spaces. Hence, $\nabla$ is a family $\nabla^{U}, U \in$ ppen $_{X}$, of $\mathbb{K}$-linear maps that commute with restrictions, and $\nabla_{\theta_{U}}^{U}, \theta_{U} \in \Theta(U)$, is a family $\left(\nabla_{\theta_{U}}^{U}\right)_{V}$, $V \in \mathrm{Open}_{U}$, of $\mathbb{K}$-linear maps that commute with restrictions. It follows that $\left.\left(\nabla_{\theta_{U}}^{U} m_{U}\right)\right|_{V}=$ $\left.\nabla_{\left.\theta_{U}\right|_{V}}^{V} m_{U}\right|_{V}$, with self-explaining notation: the concept of sheaf morphism captures the locality of the connection $\nabla$ with respect to both arguments.

Further, the requirement that the conditions (1) - (3) be satisfied for all $f \in \mathcal{O}, \theta, \theta^{\prime} \in \Theta$, and $m \in \mathcal{M}$, means that they must hold for any $U \in \operatorname{Open}_{X}$ and all $f_{U} \in \mathcal{O}(U), \theta_{U}, \theta_{U}^{\prime} \in \Theta(U)$, and $m_{U} \in \mathcal{M}(U)$.

We now detailed notation used in Proposition 1. An explanation of the underlying idea of this proposition can be found in Appendix 11.2.

\subsection{Closed symmetric monoidal structure on $\operatorname{Mod}(\mathcal{D})$}

If we apply the Hom bi-functor (resp., the tensor product bi-functor) over $\mathcal{D}$ (see (2) (resp., see (4) )) to two left $\mathcal{D}$-modules (resp., a right and a left $\mathcal{D}$-module), we get only a (sheaf of) $\mathbb{K}$-vector space(s) (see remark at the end of Section 3). The proper concept is the Hom bifunctor (resp., the tensor product bi-functor) over $\mathcal{O}$. Indeed, if $\mathcal{P}, \mathcal{Q} \in \operatorname{Mod}\left(\mathcal{D}_{X}\right) \subset \operatorname{Mod}\left(\mathcal{O}_{X}\right)$, 
the Hom sheaf $\mathcal{H o m}_{\mathcal{O}_{X}}\left(\mathcal{P}, \mathcal{Q}\right.$ ) (resp., the tensor product sheaf $\mathcal{P} \otimes_{\mathcal{O}_{X}} \mathcal{Q}$ ) is a sheaf of $\mathcal{O}_{X^{-}}$ modules. To define on this $\mathcal{O}_{X}$-module, an extending left $\mathcal{D}_{X}$-module structure, it suffices, as easily checked, to define the action of $\theta \in \Theta_{X}$ on $\phi \in \mathcal{H o m}_{\mathcal{O}_{X}}(\mathcal{P}, \mathcal{Q})$, for any $p \in \mathcal{P}$, by

$$
\left(\nabla_{\theta} \phi\right)(p)=\nabla_{\theta}(\phi(p))-\phi\left(\nabla_{\theta} p\right)
$$

( resp., on $p \otimes q, p \in \mathcal{P}, q \in \mathcal{Q}$, by

$$
\left.\nabla_{\theta}(p \otimes q)=\left(\nabla_{\theta} p\right) \otimes q+p \otimes\left(\nabla_{\theta} q\right)\right) .
$$

The functor

$$
\mathcal{H o m}_{\mathcal{O}_{X}}(\mathcal{P}, \bullet): \operatorname{Mod}\left(\mathcal{D}_{X}\right) \rightarrow \operatorname{Mod}\left(\mathcal{D}_{X}\right)
$$

$\mathcal{P} \in \operatorname{Mod}\left(\mathcal{D}_{X}\right)$, is the right adjoint of the functor

$$
\text { - } \otimes_{\mathcal{O}_{X}} \mathcal{P}: \operatorname{Mod}\left(\mathcal{D}_{X}\right) \rightarrow \operatorname{Mod}\left(\mathcal{D}_{X}\right):
$$

for any $\mathcal{N}, \mathcal{P}, \mathcal{Q} \in \operatorname{Mod}\left(\mathcal{D}_{X}\right)$, there is an isomorphism

$$
\mathcal{H}^{\circ} m_{\mathcal{D}_{X}}\left(\mathcal{N} \otimes_{\mathcal{O}_{X}} \mathcal{P}, \mathcal{Q}\right) \ni f \mapsto(n \mapsto(p \mapsto f(n \otimes p))) \in \mathcal{H o m}_{\mathcal{D}_{X}}\left(\mathcal{N}, \mathcal{H} o m_{\mathcal{O}_{X}}(\mathcal{P}, \mathcal{Q})\right)
$$

Hence, the category $\left(\operatorname{Mod}\left(\mathcal{D}_{X}\right), \otimes_{\mathcal{O}_{X}}, \mathcal{O}_{X}, \mathcal{H} m_{\mathcal{O}_{X}}\right)$ is abelian closed symmetric monoidal. More details on $\mathcal{D}$-modules can be found in [KS90, Sch12, Sch94].

Remark 2. In the following, the underlying space $X$ is a smooth algebraic variety over an algebraically closed field $\mathbb{K}$ of characteristic 0 .

We denote by $\operatorname{qc} \operatorname{Mod}\left(\mathcal{O}_{X}\right)\left(\operatorname{resp} ., \operatorname{qcMod}\left(\mathcal{D}_{X}\right)\right)$ the abelian category of quasi-coherent $\mathcal{O}_{X^{-}}$ modules (resp., $\mathcal{D}_{X}$-modules that are quasi-coherent as $\mathcal{O}_{X}$-modules [HTT08]). This category is a full subcategory of $\operatorname{Mod}\left(\mathcal{O}_{X}\right)\left(\operatorname{resp} ., \operatorname{Mod}\left(\mathcal{D}_{X}\right)\right)$. Since further the tensor product of two quasi-coherent $\mathcal{O}_{X}$-modules (resp., $\mathcal{O}_{X}$-quasi-coherent $\mathcal{D}_{X}$-modules) is again of this type, and since $\mathcal{O}_{X} \in \operatorname{qcMod}\left(O_{X}\right)\left(\operatorname{resp} ., \mathcal{O}_{X} \in \operatorname{qcMod}\left(\mathcal{D}_{X}\right)\right)$, the category $\left(\operatorname{qc} \operatorname{Mod}\left(\mathcal{O}_{X}\right), \otimes_{\mathcal{O}_{X}}, \mathcal{O}_{X}\right)$ (resp., $\left.\left(\mathrm{qc} \operatorname{Mod}\left(\mathcal{D}_{X}\right), \otimes_{\mathcal{O}_{X}}, \mathcal{O}_{X}\right)\right)$ is a symmetric monoidal subcategory of $\left(\operatorname{Mod}\left(\mathcal{O}_{X}\right), \otimes_{\mathcal{O}_{X}}, \mathcal{O}_{X}\right)$ (resp., $\left.\left(\operatorname{Mod}\left(\mathcal{D}_{X}\right), \otimes_{\mathcal{O}_{X}}, \mathcal{O}_{X}\right)\right)$. For additional information on quasi-coherent modules over a ringed space, we refer to Appendix 11.1.

\subsection{Commutative $\mathcal{D}$-algebras}

A $\mathcal{D}_{X}$-algebra is a commutative monoid in the symmetric monoidal category $\operatorname{Mod}\left(\mathcal{D}_{X}\right)$. More explicitly, a commutative $\mathcal{D}_{X}$-algebra is a $\mathcal{D}_{X}$-module $\mathcal{A}$, together with $\mathcal{D}_{X}$-linear maps

$$
\mu: \mathcal{A} \otimes_{\mathcal{O}_{X}} \mathcal{A} \rightarrow \mathcal{A} \text { and } \iota: \mathcal{O}_{X} \rightarrow \mathcal{A}
$$

which respect the usual associativity, unitality, and commutativity conditions. This means exactly that $\mathcal{A}$ is a commutative associative unital $\mathcal{O}_{X}$-algebra, which is endowed with a flat connection $\nabla$ - see Proposition 1 - such that vector fields $\theta$ act as derivations $\nabla_{\theta}$. Indeed, when omitting the latter requirement, we forget the linearity of $\mu$ and $\iota$ with respect to the 
action of vector fields. Let us translate the $\Theta_{X}$-linearity of $\mu$. If $\theta \in \Theta_{X}, a, a^{\prime} \in \mathcal{A}$, and if $a * a^{\prime}:=\mu\left(a \otimes a^{\prime}\right)$, we get

$$
\nabla_{\theta}\left(a * a^{\prime}\right)=\nabla_{\theta}\left(\mu\left(a \otimes a^{\prime}\right)\right)=\mu\left(\left(\nabla_{\theta} a\right) \otimes a^{\prime}+a \otimes\left(\nabla_{\theta} a^{\prime}\right)\right)=\left(\nabla_{\theta} a\right) * a^{\prime}+a *\left(\nabla_{\theta} a^{\prime}\right) .
$$

If we set now $1_{\mathcal{A}}:=\iota(1)$, Equation (7) shows that $\nabla_{\theta}\left(1_{\mathcal{A}}\right)=0$. It is easily checked that the $\Theta_{X}$-linearity of $\iota$ does not encode any new information. Hence,

Definition 3. A commutative $\mathcal{D}_{X}$-algebra is a commutative monoid in $\operatorname{Mod}\left(\mathcal{D}_{X}\right)$, i.e., a commutative associative unital $\mathcal{O}_{X}$-algebra that is endowed with a flat connection $\nabla$ such that $\nabla_{\theta}, \theta \in \Theta_{X}$, is a derivation.

Remark 4. All $\mathcal{D}_{X}$-algebras considered throughout this text are implicitly assumed to be commutative.

\section{Differential graded $\mathcal{D}$-modules and differential graded $\mathcal{D}$ - algebras}

\subsection{Monoidal categorical equivalence between chain complexes of $\mathcal{D}_{X^{-}}$ modules and their global sections}

It is well known that any equivalence $F: \mathrm{C} \rightleftarrows \mathrm{D}: G$ between abelian categories is exact. Moreover, if $F: \mathrm{C} \rightleftarrows \mathrm{D}: G$ is an equivalence between monoidal categories, and if one of the functors $F$ or $G$ is strong monoidal, then the other is strong monoidal as well [KRO07.

In addition, see (91), for any affine algebraic variety $X$, we have the equivalence

$$
\Gamma(X, \bullet): \operatorname{qcMod}\left(\mathcal{O}_{X}\right) \rightleftarrows \operatorname{Mod}\left(\mathcal{O}_{X}(X)\right): \widetilde{\bullet}
$$

between abelian symmetric monoidal categories, where $\boldsymbol{\bullet}$ is isomorphic to $\mathcal{O}_{X} \otimes_{\mathcal{O}_{X}(X)} \bullet$. Since the latter is obviously strong monoidal, both functors, $\Gamma(X, \bullet)$ and $\widetilde{\bullet}$, are exact and strong monoidal. Similarly,

Proposition 5. If $X$ is a smooth affine algebraic variety, its global section functor $\Gamma(X, \bullet)$ yields an equivalence

$$
\Gamma(X, \bullet):\left(\operatorname{qc} \operatorname{Mod}\left(\mathcal{D}_{X}\right), \otimes_{\mathcal{O}_{X}}, \mathcal{O}_{X}\right) \rightarrow\left(\operatorname{Mod}\left(\mathcal{D}_{X}(X)\right), \otimes_{\mathcal{O}_{X}(X)}, \mathcal{O}_{X}(X)\right)
$$

between abelian symmetric monoidal categories, and it is exact and strong monoidal.

Proof. For the categorical equivalence, see [HTT08, Proposition 1.4.4]. Exactness is now clear and it suffices to show that $\Gamma(X, \bullet)$ is strong monoidal. We know that $\Gamma(X, \bullet)$ is strong monoidal as functor between modules over functions, see (8). Hence, if $\mathcal{P}, \mathcal{Q} \in \operatorname{qcMod}\left(\mathcal{D}_{X}\right)$, then

$$
\Gamma\left(X, \mathcal{P} \otimes_{\mathcal{O}_{X}} \mathcal{Q}\right) \simeq \Gamma(X, \mathcal{P}) \otimes_{\mathcal{O}_{X}(X)} \Gamma(X, \mathcal{Q})
$$


as $\mathcal{O}_{X}(X)$-modules. Recall now that we defined the $\mathcal{D}_{X}$-module structure on $\mathcal{P} \otimes_{\mathcal{O}_{X}} \mathcal{Q}$ by 'extending' the $\Theta_{X}$-action (6) on the presheaf $\mathcal{P} \oslash_{\mathcal{O}_{X}} \mathcal{Q}$, see (3). In view of (10), the action $\nabla^{X}$ of $\Theta_{X}(X)$ on $\mathcal{P}(X) \otimes_{\mathcal{O}_{X}(X)} \mathcal{Q}(X)$ and on $\left(\mathcal{P} \otimes_{\mathcal{O}_{X}} \mathcal{Q}\right)(X)$ 'coincide', and so do the $\mathcal{D}_{X}(X)$ module structures of these modules. Finally, the global section functor is strong monoidal.

Remark 6. In the following, we work systematically over a smooth affine algebraic variety $X$ over an algebraically closed field $\mathbb{K}$ of characteristic 0.

Since the category $\operatorname{qcMod}\left(\mathcal{D}_{X}\right)$ is abelian symmetric monoidal, the category $\mathrm{DG}_{+} \mathrm{qcMod}\left(\mathcal{D}_{X}\right)$ of differential non-negatively graded $\mathcal{O}_{X}$-quasi-coherent $\mathcal{D}_{X}$-modules is abelian and symmetric monoidal as well - for the usual tensor product of chain complexes and chain maps. The unit of this tensor product is the chain complex $\mathcal{O}_{X}$ concentrated in degree 0 . The symmetry $\beta: \mathcal{P}_{\bullet} \otimes \mathcal{Q}_{\bullet} \rightarrow \mathcal{Q}_{\bullet} \otimes \mathcal{P}_{\bullet}$ is given by

$$
\beta(p \otimes q)=(-1)^{\tilde{p} \tilde{q}} q \otimes p,
$$

where 'tilde' denotes the degree and where the sign is necessary to obtain a chain map. Let us also mention that the zero object of $\mathrm{DG}_{+} \mathrm{qc} \operatorname{Mod}\left(\mathcal{D}_{X}\right)$ is the chain complex $(\{0\}, 0)$.

Proposition 7. If $X$ is a smooth affine algebraic variety, its global section functor induces an equivalence

$$
\Gamma(X, \bullet):\left(\mathrm{DG}_{+} \mathrm{qcMod}\left(\mathcal{D}_{X}\right), \otimes_{\mathcal{O}_{X}}, \mathcal{O}_{X}\right) \rightarrow\left(\mathrm{DG}_{+} \operatorname{Mod}\left(\mathcal{D}_{X}(X)\right), \otimes_{\mathcal{O}_{X}(X)}, \mathcal{O}_{X}(X)\right)
$$

of abelian symmetric monoidal categories, and is exact and strong monoidal.

Proof. Let $F=\Gamma(X, \bullet)$ and $G$ be quasi-inverse (additive) functors that implement the equivalence (9). They induce functors $\mathbf{F}$ and $\mathbf{G}$ between the corresponding categories of chain complexes. Moreover, the natural isomorphism $a: \mathrm{id} \Rightarrow G \circ F$ induces, for each chain complex $\mathcal{P}_{\bullet} \in \operatorname{DG}_{+} \operatorname{qc} \operatorname{Mod}\left(\mathcal{D}_{X}\right)$, a chain isomorphism $\mathbf{a}_{\mathcal{P}_{\bullet}}: \mathcal{P}_{\bullet} \rightarrow(\mathbf{G} \circ \mathbf{F})\left(\mathcal{P}_{\bullet}\right)$, which is functorial in $\mathcal{P}_{\bullet}$. Both, the chain morphism property of $\mathbf{a}_{\mathcal{P}_{\bullet}}$ and the naturality of $\mathbf{a}$, are direct consequences of the naturality of $a$ - since the action of $\mathbf{a}$ on a chain complex is given by the degreewise action of $a$. Similarly, the natural isomorphism $b: F \circ G \Rightarrow$ id induces a natural isomorphism $\mathbf{b}: \mathbf{F} \circ \mathbf{G} \Rightarrow$ id, so that $\mathrm{DG}_{+} \mathrm{qc} \operatorname{Mod}\left(\mathcal{D}_{X}\right)$ and $\mathrm{DG}_{+} \operatorname{Mod}\left(\mathcal{D}_{X}(X)\right)$ are actually equivalent categories. Since $F: \operatorname{qc} \operatorname{Mod}\left(\mathcal{D}_{X}\right) \rightarrow \operatorname{Mod}\left(\mathcal{D}_{X}(X)\right)$ is strong monoidal and commutes with colimits (as left adjoint of $G$ ), it is straightforwardly checked that $\mathbf{F}$ is strong monoidal.

\subsection{Differential graded $\mathcal{D}_{X}$-algebras vs. differential graded $\mathcal{D}_{X}(X)$-algebras}

The strong monoidal functors $\mathbf{F}: \mathrm{DG}_{+} \mathrm{qcMod}\left(\mathcal{D}_{X}\right) \rightleftarrows \mathrm{DG}_{+} \operatorname{Mod}\left(\mathcal{D}_{X}(X)\right): \mathbf{G}$ yield an equivalence between the corresponding categories of commutative monoids:

Corollary 8. For any smooth affine variety $X$, there is an equivalence of categories

$$
\Gamma(X, \bullet): \mathrm{DG}_{+} \mathrm{qcCAlg}\left(\mathcal{D}_{X}\right) \rightarrow \mathrm{DG}_{+} \operatorname{CAlg}\left(\mathcal{D}_{X}(X)\right)
$$

between the category of differential graded quasi-coherent commutative $\mathcal{D}_{X}$-algebras and the category of differential graded commutative $\mathcal{D}_{X}(X)$-algebras. 
The main goal of the present paper is to construct a model category structure on the LHS category. In view of the preceding corollary, it suffices to build this model structure on the RHS category. We thus deal in the following exclusively with the category of differential graded $\mathcal{D}$-algebras (resp., $\mathcal{D}$-algebras), where $\mathcal{D}:=\mathcal{D}_{X}(X)$, which we denote simply by DGDA (resp., $\mathcal{D} A)$. Similarly, the objects of $\mathrm{DG}_{+} \operatorname{Mod}\left(\mathcal{D}_{X}(X)\right)$ (resp., $\operatorname{Mod}\left(\mathcal{D}_{X}(X)\right)$ ) are termed differential graded $\mathcal{D}$-modules (resp., $\mathcal{D}$-modules) and their category is denoted by DGDM (resp., $\mathcal{D} \mathrm{M})$.

\subsection{The category DGDA}

In this subsection we describe the category DGDA and prove first properties.

Whereas $\operatorname{Hom}_{\mathcal{D M}}(P, Q), P, Q \in \mathcal{D} \mathrm{M}$, is a $\mathbb{K}$-vector space, the set $\operatorname{Hom}_{\mathcal{D A}}(A, B), A, B \in \mathcal{D} \mathrm{A}$, is not even an abelian group. Hence, we cannot consider the category of chain complexes over commutative $\mathcal{D}$-algebras and the objects of DGDA are (probably useless to say) no chain complexes of algebras.

As explained above, a $\mathcal{D}$-algebra is a commutative unital $\mathcal{O}$-algebra, endowed with a $\mathcal{D}$ module structure (which extends the $\mathcal{O}$-module structure), such that vector fields act by derivations. Analogously, a differential graded $\mathcal{D}$-algebra is easily seen to be a differential graded commutative unital $\mathcal{O}$-algebra (a graded $\mathcal{O}$-module together with an $\mathcal{O}$-bilinear degree respecting multiplication, which is associative, unital, and graded-commutative; this module comes with a square 0 , degree $-1, \mathcal{O}$-linear, graded derivation), which is also a differential graded $\mathcal{D}$-module (for the same differential, grading, and $\mathcal{O}$-action), such that vector fields act as degree zero derivations.

Proposition 9. A differential graded $\mathcal{D}$-algebra is a differential graded commutative unital $\mathcal{O}$-algebra, as well as a differential graded $\mathcal{D}$-module, such that vector fields act as derivations. Further, the morphisms of DGDA are the morphisms of DGDM that respect the multiplications and units.

In fact:

Proposition 10. The category DGDA is symmetric monoidal for the tensor product of DGDM with values on objects that are promoted canonically from DGDM to DGDA and same values on morphisms. The tensor unit is $\mathcal{O}$; the initial object ( resp., terminal object) is $\mathcal{O}$ (resp., $\{0\}$ ).

Proof. Let $A_{\bullet}, B_{\bullet} \in \mathrm{DGDA}$. Consider homogeneous vectors $a \in A_{\tilde{a}}, a^{\prime} \in A_{\tilde{a}^{\prime}}, b \in B_{\tilde{b}}, b^{\prime} \in B_{\tilde{b}^{\prime}}$, such that $\tilde{a}+\tilde{b}=m$ and $\tilde{a}^{\prime}+\tilde{b}^{\prime}=n$. Endow now the tensor product $A_{\bullet} \otimes_{\mathcal{O}} B_{\bullet} \in$ DGDM with the multiplication $\star$ defined by

$$
\begin{gathered}
\left(A_{\bullet} \otimes_{\mathcal{O}} B_{\bullet}\right)_{m} \times\left(A_{\bullet} \otimes_{\mathcal{O}} B_{\bullet}\right)_{n} \ni\left(a \otimes b, a^{\prime} \otimes b^{\prime}\right) \mapsto \\
(a \otimes b) \star\left(a^{\prime} \otimes b^{\prime}\right)=(-1)^{\tilde{a}^{\prime} \tilde{b}}\left(a \star_{A} a^{\prime}\right) \otimes\left(b \star_{B} b^{\prime}\right) \in\left(A_{\bullet} \otimes_{\mathcal{O}} B_{\bullet}\right)_{m+n},
\end{gathered}
$$

where the multiplications of $A_{\bullet}$ and $B_{\bullet}$ are denoted by $\star_{A}$ and $\star_{B}$, respectively. The multiplication $\star$ equips $A_{\bullet} \otimes_{\mathcal{O}} B_{\bullet}$ with a structure of differential graded $\mathcal{D}$-algebra. Note also that the multiplication of $A_{\bullet} \in \mathrm{DGDA}$ is a DGDA-morphism $\mu_{A}: A_{\bullet} \otimes_{\mathcal{O}} A_{\bullet} \rightarrow A_{\bullet}$. 
Further, the unit of the tensor product in $\mathrm{DGDA}$ is the unit $(\mathcal{O}, 0)$ of the tensor product in DGDM.

Finally, let $A_{\bullet}, B_{\bullet}, C_{\bullet}, D_{\bullet} \in \mathrm{DGDA}$ and let $\phi: A_{\bullet} \rightarrow C_{\bullet}$ and $\psi: B_{\bullet} \rightarrow D_{\bullet}$ be two DGDAmorphisms. Then the DGDM-morphism $\phi \otimes \psi: A_{\bullet} \otimes_{\mathcal{O}} B_{\bullet} \rightarrow C_{\bullet} \otimes_{\mathcal{O}} D_{\bullet}$ is also a DGDA-morphism.

All these claims (as well as all the additional requirements for a symmetric monoidal structure) are straightforwardly checked.

The initial and terminal objects in DGDA are the differential graded $\mathcal{D}$-algebras $(\mathcal{O}, 0)$ and $(\{0\}, 0)$, respectively. Indeed, in view of the adjunction (18), the initial object of DGDA is the image by $\mathcal{S}$ of the initial object of DGDM.

Let us still mention the following

Proposition 11. If $\phi: A_{\bullet} \rightarrow C_{\bullet}$ and $\psi: B_{\bullet} \rightarrow C_{\bullet}$ are DGDA-morphisms, then $\chi: A_{\bullet} \otimes_{\mathcal{O}} B_{\bullet} \rightarrow$ $C_{\bullet}$, which is well-defined by $\chi(a \otimes b)=\phi(a){ }_{\star_{C}} \psi(b)$, is a DGDA-morphism that restricts to $\phi$ (resp., $\psi)$ on $A_{\bullet}\left(\right.$ resp., $\left.B_{\bullet}\right)$.

Proof. It suffices to observe that $\chi=\mu_{C} \circ(\phi \otimes \psi)$.

\section{$6 \quad$ Finitely generated model structure on DGDM}

When dealing with model categories, we use the definitions of [Hov07]. A short comparison of various definitions used in the literature can be found in Appendix 11.4 below. For additional information, we refer the reader to GS06, Hir00, Hov07, and Qui67.

Let us recall that $\mathrm{DGDM}$ is the category $\mathrm{Ch}_{+}(\mathcal{D})$ of non-negatively graded chain complexes of left modules over the non-commutative unital ring $\mathcal{D}=\mathcal{D}_{X}(X)$ of differential operators of a smooth affine algebraic variety $X$. The remaining part of this section actually holds for any not necessarily commutative unital ring $R$ and the corresponding category $\mathrm{Ch}_{+}(R)$. We will show that $\mathrm{Ch}_{+}(R)$ is a finitely (and thus cofibrantly) generated model category.

In fact, most of the familiar model categories are cofibrantly generated. For instance, in the model category SSet of simplicial sets, the generating cofibrations $I$ (resp., the generating trivial cofibrations $J$ ) are the canonical simplicial maps $\partial \Delta[n] \rightarrow \Delta[n]$, whose sources are the boundaries of the standard simplicial $n$-simplices (resp., the canonical maps $\Lambda^{r}[n] \rightarrow \Delta[n]$, whose sources are the $r$-horns of the standard $n$-simplices, $0 \leq r \leq n)$. The generating cofibrations and trivial cofibrations of the model category Top of topological spaces - which is Quillen equivalent to SSet - are defined similarly. The homological situation is analogous to the topological and combinatorial ones. In the case of $\mathrm{Ch}_{+}(R)$, the set $I$ of generating cofibrations (resp., the set $J$ of generating trivial cofibrations) is made (roughly) of the maps $S^{n-1} \rightarrow D^{n}$ from the $(n-1)$-sphere to the $n$-disc (resp., of the maps $\left.0 \rightarrow D^{n}\right)$. In fact, the $n$-disc $D^{n}$ is the chain complex

$$
D_{\bullet}^{n}: \cdots \rightarrow 0 \rightarrow 0 \rightarrow \stackrel{(n)}{R} \rightarrow \stackrel{(n-1)}{R} \rightarrow 0 \rightarrow \cdots \rightarrow \stackrel{(0)}{0},
$$


whereas the $n$-sphere $S^{n}$ is the chain complex

$$
S_{\bullet}^{n}: \cdots \rightarrow 0 \rightarrow 0 \rightarrow \stackrel{(n)}{R} \rightarrow 0 \rightarrow \cdots \rightarrow \stackrel{(0)}{0} .
$$

Definition (14), in which the differential is necessarily the identity of $R$, is valid for $n \geq 1$. Definition (15) makes sense for $n \geq 0$. We extend the first (resp., second) definition to $n=0$ (resp., $n=-1$ ) by setting $D_{\bullet}^{0}:=S_{\bullet}^{0}\left(\right.$ resp., $\left.S_{\bullet}^{-1}:=0_{\bullet}\right)$. The chain maps $S^{n-1} \rightarrow D^{n}$ are canonical (in degree $n-1$, they necessarily coincide with $\operatorname{id}_{R}$ ), and so are the maps $0 \rightarrow D^{n}$. We now define the set $I$ (resp., $J$ ) by

$$
I=\left\{\iota_{n}: S^{n-1} \rightarrow D^{n}, n \geq 0\right\}
$$

(resp.,

$$
\left.J=\left\{\zeta_{n}: 0 \rightarrow D^{n}, n \geq 1\right\}\right) .
$$

Theorem 12. For any unital ring $R$, the category $\mathrm{Ch}_{+}(R)$ of non-negatively graded chain complexes of left $R$-modules is a finitely (and thus a cofibrantly) generated model category (in the sense of [GS06] and in the sense of [Hov07]), with I as its generating set of cofibrations and $J$ as its generating set of trivial cofibrations. The weak equivalences are the maps that induce isomorphisms in homology, the cofibrations are the injective maps with degree-wise projective cokernel ( projective object in $\operatorname{Mod}(R))$, and the fibrations are the maps that are surjective in (strictly) positive degrees. Further, the trivial cofibrations are the injective maps $i$ whose cokernel coker $(i)$ is strongly projective as a chain complex (strongly projective object coker $(i)$ in $\mathrm{Ch}_{+}(R)$, in the sense that, for any map $c: \operatorname{coker}(i) \rightarrow C$ and any map $p: D \rightarrow C$, there is a map $\ell: \operatorname{coker}(i) \rightarrow D$ such that $p \circ \ell=i$, if $p$ is surjective in (strictly) positive degrees).

Proof. The following proof uses the definitions of (cofibrantly generated) model categories used in [DS96] and [GS06], as well as the non-equivalent definitions of these concepts given in [Hov07]: we refer again to the Appendix 11.4 below.

It is known that $\mathrm{Ch}_{+}(R)$, with the described weak equivalences, cofibrations, and fibrations is a model category (Theorem 7.2 in [DS96]). A model category in the sense of [DS96] contains all finite limits and colimits; the Cof - TrivFib and TrivCof - Fib factorizations are neither assumed to be functorial, nor, of course, to be chosen functorial factorizations. Moreover, we have Fib $=\operatorname{RLP}(J)$ and TrivFib $=\operatorname{RLP}(I)$ (Proposition 7.19 in [DS96]).

Note first that $\mathrm{Ch}_{+}(R)$ has all small limits and colimits, which are taken degree-wise.

Observe also that the domains and codomains $S^{n}(n \geq 0)$ and $D^{n}(n \geq 1)$ of the maps in $I$ and $J$ are bounded chain complexes of finitely presented $R$-modules (the involved modules are all equal to $R$ ). However, every bounded chain complex of finitely presented $R$-modules is $n$-small, $n \in \mathbb{N}$, relative to all chain maps (Lemma 2.3.2 in [Hov07]). Hence, the domains and codomains of $I$ and $J$ satisfy the smallness condition of a finitely generated model category, and are therefore small in the sense of the finite and transfinite definitions of a cofibrantly generated model category. 
It thus follows from the Small Object Argument that there exist in $\mathrm{Ch}_{+}(R)$ a functorial Cof - TrivFib and a functorial TrivCof - Fib factorization. Hence, the first part of Theorem 12.

As for the part on trivial cofibrations, its proof is the same as the proof of Lemma 2.2.11 in [Hov07].

In view of Theorem [12, let us recall that any projective chain complex $(K, d)$ is degree-wise projective. Indeed, consider, for $n \geq 0$, an $R$-linear map $k_{n}: K_{n} \rightarrow N$ and a surjective $R$-linear map $p: M \rightarrow N$, and denote by $D^{n+1}(N)$ (resp., $D^{n+1}(M)$ ) the disc defined as in (14)), except that $R$ is replaced by $N$ (resp., $M$ ). Then there is a chain map $k: K \rightarrow D^{n+1}(N)$ (resp., a surjective chain map $\left.\pi: D^{n+1}(M) \rightarrow D^{n+1}(N)\right)$ that is zero in each degree, except in degree $n+1$ where it is $k_{n} \circ d_{n+1}$ (resp., $p$ ) and in degree $n$ where it is $k_{n}$ (resp., $p$ ). Since $(K, d)$ is projective as chain complex, there is a chain map $\ell: K \rightarrow D^{n+1}(M)$ such that $\pi \circ \ell=k$. In particular, $\ell_{n}: K_{n} \rightarrow M$ is $R$-linear and $p \circ \ell_{n}=k_{n}$.

\section{Finitely generated model structure on DGDA}

\subsection{Adjoint functors between DGDM and DGDA}

We aim at transferring to DGDA the just described finitely generated model structure on DGDM. Therefore, we need a pair of adjoint functors.

Proposition 13. The graded symmetric tensor algebra functor $\mathcal{S}$ and the forgetful functor For provide an adjoint pair

$$
\mathcal{S}: \mathrm{DGDM} \rightleftarrows \mathrm{DGDA}: \text { For }
$$

between the category of differential graded $\mathcal{D}$-modules and the category of differential graded $\mathcal{D}$-algebras.

Proof. For any $M_{\bullet} \in \mathrm{DGDM}$, the sum

$$
\otimes_{\mathcal{O}}^{*} M_{\bullet}=\mathcal{O} \oplus \bigoplus_{n \geq 1} M_{\bullet}^{\otimes \mathcal{O}} n \in \mathrm{DGDM}
$$

is the free associative unital $\mathcal{O}$-algebra over the $\mathcal{O}$-module $M_{\bullet}$. When passing to graded symmetric tensors, we divide by the obvious $\mathcal{O}$-ideal $\mathcal{I}$, which is further a sub DG $\mathcal{D}$-module. Therefore, the free graded symmetric unital $\mathcal{O}$-algebra

$$
\mathcal{S}_{\mathcal{O}}^{*} M_{\bullet}=\otimes_{\mathcal{O}}^{*} M_{\bullet} / \mathcal{I}
$$

with multiplication $[S] \odot[T]=[S \otimes T]$, is also a DG $\mathcal{D}$-module. It is straightforwardly checked that $\mathcal{S}_{\mathcal{O}}^{*} M_{\bullet} \in \mathrm{DGDA}$. The definition of $\mathcal{S}$ on morphisms is obvious.

As concerns the proof that the functors For and $\mathcal{S}$ are adjoint, i.e., that

$$
\operatorname{Hom}_{\mathrm{DG} \mathcal{D A}}\left(\mathcal{S}_{\mathcal{O}}^{*} M_{\bullet}, A_{\bullet}\right) \simeq \operatorname{Hom}_{\mathrm{DG} \mathcal{D M}}\left(M_{\bullet}, \text { For } A_{\bullet}\right)
$$


functorially in $M_{\bullet} \in \mathrm{DGDM}$ and $A_{\bullet} \in \mathrm{DGDA}$, let $\phi: M_{\bullet} \rightarrow$ For $A_{\bullet}$ be a DGDM-map. Since $\mathcal{S}_{\mathcal{O}}^{*} M_{\bullet}$ is free in the category GCA of graded commutative associative unital graded $\mathcal{O}$-algebras, a GCAmorphism is completely determined by its restriction to the graded $\mathcal{O}$-module $M_{\bullet}$. Hence, the extension $\bar{\phi}: \mathcal{S}_{\mathcal{O}}^{*} M_{\bullet} \rightarrow A_{\bullet}$ of $\phi$, defined by $\bar{\phi}\left(1_{\mathcal{O}}\right)=1_{A}$ and by

$$
\bar{\phi}\left(m_{1} \odot \ldots \odot m_{k}\right)=\phi\left(m_{1}\right) \star_{A} \ldots \star_{A} \phi\left(m_{k}\right),
$$

is a GCA-morphism. This extension is also a DGDA-map, i.e., a DGDM-map that respects the multiplications and the units, if it intertwines the differentials and is $\mathcal{D}$-linear. These requirements, as well as functoriality, are straightforwardly checked.

Recall that a free object in a category $\mathrm{D}$ over an object $C$ in a category $\mathrm{C}$, such that there is a forgetful functor For $: \mathrm{D} \rightarrow \mathrm{C}$, is a universal pair $(F(C), i)$, where $F(C) \in \mathrm{D}$ and $i \in \operatorname{Hom}_{\mathrm{C}}(C$, For $F(C))$.

Remark 14. Equation (20) means that $\mathcal{S}_{\mathcal{O}}^{\star} M_{\bullet}$ is the free differential graded $\mathcal{D}$-algebra over the differential graded $\mathcal{D}$-module $M_{\bullet}$.

A definition of $\mathcal{S}_{\mathcal{O}}^{*} M_{\bullet}$ via invariants can be found in Appendix 11.5.

\subsection{Relative Sullivan $\mathcal{D}$-algebras}

If $V_{\bullet}$ is a non-negatively graded $\mathcal{D}$-module and $\left(A_{\bullet}, d_{A}\right)$ a differential graded $\mathcal{D}$-algebra, the tensor product $A_{\bullet} \otimes_{\mathcal{O}} \mathcal{S}_{\mathcal{O}}^{\star} V_{\bullet}$ is a graded $\mathcal{D}$-algebra. In the following definition, we assume that this algebra is equipped with a differential $d$, such that

$$
\left(A_{\bullet} \otimes_{\mathcal{O}} \mathcal{S}_{\mathcal{O}}^{\star} V_{\bullet}, d\right) \in \mathrm{DGDA}
$$

contains $\left(A_{\bullet}, d_{A}\right)$ as sub-DGDA. The point is that $\left(A_{\bullet}, d_{A}\right)$ is a differential submodule of the tensor product differential module, but that usually the module $\mathcal{S}_{\mathcal{O}}^{\star} V_{\bullet}$ is not. The condition that $\left(A_{\bullet}, d_{A}\right)$ be a sub-DGDA can be rephrased by asking that the inclusion

$$
A_{\bullet} \ni a \mapsto a \otimes 1 \in A_{\bullet} \otimes_{\mathcal{O}} \mathcal{S}_{\mathcal{O}}^{\star} V_{\bullet}
$$

be a DGDA-morphism. This algebra morphism condition or subalgebra condition would be automatically satisfied if the differential $d$ on $A \bullet \otimes_{\mathcal{O}} \mathcal{S}_{\mathcal{O}}^{\star} V \bullet$ were defined by

$$
d=d_{A} \otimes \mathrm{id}+\mathrm{id} \otimes d_{\mathcal{S}},
$$

where $d_{\mathcal{S}}$ is a differential on $\mathcal{S}_{\mathcal{O}}^{\star} V_{\bullet}$ (in particular the differential $d_{\mathcal{S}}=0$ ). However, as mentioned, this is generally not the case.

We omit in the following $\bullet$, $\star$, as well as subscript $\mathcal{O}$, provided clarity does not suffer hereof. Further, to avoid confusion, we sometimes substitute $\otimes$ for $\otimes$ to emphasize that the differential $d$ of $A \otimes \mathcal{S} V$ is not necessarily obtained from the differential $d_{A}$ and a differential $d_{\mathcal{S}} 1$

\footnotetext{
${ }^{1}$ Such twisted differentials typically appear when one adds new generators to improve homological properties and in particular to kill homology in lower degrees.
} 
We now give the $\mathcal{D}$-algebraic version of the definition of a relative Sullivan algebra [FHT01]. Note that the factorizations that are considered in [FHT01] are not, as the factorizations here below, obtained via pushouts and are not functorial.

Definition 15. A relative Sullivan $\mathcal{D}$-algebra $(R S D A)$ is a DGDA-morphism

$$
\left(A, d_{A}\right) \rightarrow(A \otimes \mathcal{S} V, d)
$$

that sends $a \in A$ to $a \otimes 1 \in A \otimes \mathcal{S} V$. Here $V$ is a free non-negatively graded $\mathcal{D}$-module

$$
V=\bigoplus_{\alpha \in J} \mathcal{D} \cdot v_{\alpha}
$$

which admits a homogeneous basis $\left(v_{\alpha}\right)_{\alpha \in J}$ that is indexed by a well-ordered set $J$, and is such that

$$
d v_{\alpha} \in A \otimes \mathcal{S} V_{<\alpha},
$$

for all $\alpha \in J$. In the last requirement, we set $V_{<\alpha}:=\bigoplus_{\beta<\alpha} \mathcal{D} \cdot v_{\beta}$. We refer to Property (22) by saying that $d$ is lowering.

A RSDA with Property (21) ( resp., over $\left(A, d_{A}\right)=(\mathcal{O}, 0)$ ) is called a split RSDA (resp., a Sullivan $\mathcal{D}$-algebra $(S \mathcal{D} A))$ and it is often simply denoted by $(A \otimes \mathcal{S} V, d)($ resp., $(\mathcal{S} V, d))$.

The next two lemmas are of interest for the split situation.

Lemma 16. Let $\left(v_{\alpha}\right)_{\alpha \in I}$ be a family of generators of homogeneous non-negative degrees, and let

$$
V:=\left\langle v_{\alpha}: \alpha \in I\right\rangle:=\bigoplus_{\alpha \in I} \mathcal{D} \cdot v_{\alpha}
$$

be the free non-negatively graded $\mathcal{D}$-module over $\left(v_{\alpha}\right)_{\alpha \in I}$. Then, any degree -1 map $d \in$ $\operatorname{Set}\left(\left(v_{\alpha}\right), V\right)$ uniquely extends to a degree -1 map $d \in \mathcal{D} \mathrm{M}(V, V)$. If moreover $d^{2}=0$ on $\left(v_{\alpha}\right)$, then $(V, d) \in \mathrm{DGDM}$.

Since $\mathcal{S} V$ is the free differential graded $\mathcal{D}$-algebra over the differential graded $\mathcal{D}$-module $V$, a morphism $f \in \operatorname{DGDA}(\mathcal{S} V, B)$, valued in $\left(B, d_{B}\right) \in \mathrm{DGDA}$, is completely defined by its restriction $f \in \operatorname{DGDM}(V, B)$. Hence, the

Lemma 17. Consider the situation of Lemma 16. Any degree 0 map $f \in \operatorname{Set}\left(\left(v_{\alpha}\right), B\right)$ uniquely extends to a morphism $f \in \mathrm{GDM}(V, B)$. Furthermore, if $d_{B} f=f d$ on $\left(v_{\alpha}\right)$, this extension is a morphism $f \in \operatorname{DGD} \mathrm{M}(V, B)$, which in turn admits a unique extension $f \in \operatorname{DGDA}(\mathcal{S} V, B)$.

\subsection{Quillen's transfer theorem}

We use the adjoint pair

$$
\mathcal{S}: \mathrm{DG} \mathcal{D M} \rightleftarrows \mathrm{DG} \mathcal{D} A: \text { For }
$$

to transfer the cofibrantly generated model structure from the source category DGDM to the target category DGDA. This is possible if Quillen's transfer theorem Qui67 applies. 
Theorem 18. Let $F: \mathrm{C} \rightleftarrows \mathrm{D}: G$ be a pair of adjoint functors. Assume that $\mathrm{C}$ is a cofibrantly generated model category and denote by I (resp., J) its set of generating cofibrations (resp., trivial cofibrations). Define a morphism $f: X \rightarrow Y$ in D to be a weak equivalence (resp., a fibration), if $G f$ is a weak equivalence (resp., a fibration) in $\mathrm{C}$. If

1. the right adjoint $G: \mathrm{D} \rightarrow \mathrm{C}$ commutes with sequential colimits, and

2. any map in $\mathrm{D}$ with the LLP with respect to all fibrations is a weak equivalence,

then $\mathrm{D}$ is a cofibrantly generated model category that admits $\{F i: i \in I\}$ (resp., $\{F j: j \in J\}$ ) as set of generating cofibrations (resp., trivial cofibrations).

Of course, in this version of the transfer principle, the mentioned model structures are cofibrantly generated model structures in the sense of [GS06].

Condition 2 is the main requirement of the transfer theorem. It can be checked using the following lemma Qui67:

Lemma 19 (Quillen's path object argument). Assume in a category D (which is not yet a model category, but has weak equivalences and fibrations),

1. there is a functorial fibrant replacement functor, and

2. every object has a natural path object, i.e., for any $D \in \mathrm{D}$, we have a natural commutative diagram

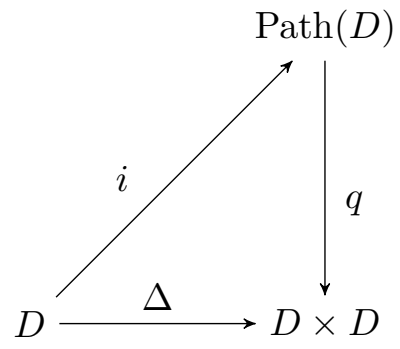

where $\Delta$ is the diagonal map, $i$ is a weak equivalence and $q$ is a fibration. Then every map in D with the LLP with respect to all fibrations is a weak equivalence.

We think about $\operatorname{Path}(D) \in \mathrm{D}$ is an internalized 'space' of paths in $D$. In simple cases, $\operatorname{Path}(D)=\operatorname{Hom}_{\mathrm{D}}(I, D)$, where $I \in \mathrm{D}$ and where $\mathrm{Hom}_{\mathrm{D}}$ is an internal Hom. Moreover, by fibrant replacement of an object $D \in \mathrm{D}$, we mean a weak equivalence $D \rightarrow \bar{D}$ whose target is a fibrant object.

\subsection{Proof of Condition 1 of Theorem 18}

Let $\lambda$ be a non-zero ordinal and let $X: \lambda \rightarrow \mathrm{C}$ be a diagram of type $\lambda$ in a category C, i.e., a functor from $\lambda$ to C. Since an ordinal number is a totally ordered set, the considered ordinal 
$\lambda$ can be viewed as a directed poset $(\lambda, \leq)$. Moreover, the diagram $X$ is a direct system in $\mathrm{C}$ over $\lambda$-made of the C-objects $X_{\beta}, \beta<\lambda$, and the C-morphisms $X_{\beta \gamma}: X_{\beta} \rightarrow X_{\gamma}, \beta \leq \gamma$, and the colimit $\operatorname{colim}_{\beta<\lambda} X_{\beta}$ of this diagram $X$ is the inductive limit to the system $\left(X_{\beta}, X_{\beta \gamma}\right)$.

Let now $A: \lambda \rightarrow \mathrm{DGDA}$ be a diagram of type $\lambda$ in DGDA and let For $\circ A: \lambda \rightarrow \mathrm{DGDM}$ be the corresponding diagram in DGDM. To simplify notation, we denote the latter diagram simply by $A$. As mentioned in the proof of Theorem 12, the colimit of $A$ does exist in DGDM and is taken degree-wise in $\operatorname{Mod}(\mathcal{D})$. For any degree $r \in \mathbb{N}$, the colimit $C_{r}$ of the functor $A_{r}: \lambda \rightarrow \operatorname{Mod}(\mathcal{D})$ is the inductive limit in $\operatorname{Mod}(\mathcal{D})$ to the direct system $\left(A_{\beta, r}, A_{\beta \gamma, r}\right)$ - which is obtained via the usual construction in Set. Due to universality, one naturally gets a $\operatorname{Mod}(\mathcal{D})$-morphism $d_{r}: C_{r} \rightarrow C_{r-1}$. The complex $\left(C_{\bullet}, d\right)$ is the colimit in DGDM of $A$. It is now straightforwardly checked that the canonical multiplication $\diamond$ in $C_{\bullet}$ provides an object $\left(C_{\bullet}, d, \diamond\right) \in \mathrm{DGDA}$ and that this object is the colimit of $A$ in DGDA.

Hence, the

Proposition 20. Let $\lambda$ be a non-zero ordinal. The forgetful functor For : DGDA $\rightarrow$ DGDM creates colimits of diagrams of type $\lambda$ in $\mathrm{DGDA}$, i.e., for any diagram $A$ of type $\lambda$ in $\mathrm{DGDA}$, we have

$$
\operatorname{For}\left(\operatorname{colim}_{\beta<\lambda} A_{\beta, \bullet}\right)=\operatorname{colim}_{\beta<\lambda} \operatorname{For}\left(A_{\beta, \bullet}\right) .
$$

If $\lambda$ is the zero ordinal, it can be viewed as the empty category $\emptyset$. Therefore, the colimit in DGDA of the diagram of type $\lambda$ is in this case the initial object $(\mathcal{O}, 0)$ of DGDA. Since the initial object in DGDM is $(\{0\}, 0)$, we see that For does not commute with this colimit. The above proof fails indeed, as $\emptyset$ is not a directed set.

It follows from Proposition 20 that the right adjoint For in (23) commutes with sequential colimits, so that the first condition of Theorem 18 is satisfied.

Remark 21. Since a right adjoint functor between accessible categories preserves all filtered colimits, the first condition of Theorem 18 is a consequence of the accessibility of DGDM and DGDA. We gave a direct proof to avoid the proof of the accessibility of DGDA.

\subsection{Proof of Condition 2 of Theorem 18}

We prove Condition 2 using Lemma 19, In our case, the adjoint pair is

$$
\mathcal{S}: \mathrm{DGDM} \rightleftarrows \mathrm{DGDA}: \text { For } .
$$

As announced in Subsection 7.2, we omit $\bullet$, $\star$, and $\mathcal{O}$, whenever possible. It is clear that every object $A \in \mathrm{D}=\mathrm{DGDA}$ is fibrant. Hence, we can choose the identity as fibrant replacement functor, with the result that the latter is functorial.

As for the second condition of the lemma, we will show that any DGDA-morphism $\phi: A \rightarrow B$ naturally factors into a weak equivalence followed by a fibration.

Since in the standard model structure on the category of differential graded commutative algebras over $\mathbb{Q}$, cofibrations are retracts of relative Sullivan algebras [Hes00], the obvious idea 
is to decompose $\phi$ as $A \rightarrow A \otimes \mathcal{S} V \rightarrow B$, where $i: A \rightarrow A \otimes \mathcal{S} V$ is a (split) relative Sullivan $\mathcal{D}$-algebra, such that there is a projection $p: A \otimes \mathcal{S} V \rightarrow B$, or, even better, a projection $\varepsilon: V \rightarrow B$ in positive degrees. The first attempt might then be to use

$$
\varepsilon: V=\bigoplus_{n>0} \bigoplus_{b_{n} \in B_{n}} \mathcal{D} \cdot 1_{b_{n}} \ni 1_{b_{n}} \mapsto b_{n} \in B
$$

whose source incorporates a copy of the sphere $S^{n}$ for each $b_{n} \in B_{n}, n>0$. However, $\varepsilon$ is not a chain map, since in this case we would have $d_{B} b_{n}=d_{B} \varepsilon 1_{b_{n}}=0$, for all $b_{n}$. The next candidate is obtained by replacing $S^{n}$ by $D^{n}$ : if $B \in \mathrm{DGDM}$, set

$$
P(B)=\bigoplus_{n>0} \bigoplus_{b_{n} \in B_{n}} D_{\bullet}^{n} \in \mathrm{DGDM}
$$

where $D_{\bullet}^{n}$ is a copy of the $n$-disc

$$
D_{\bullet}^{n}: \cdots \rightarrow 0 \rightarrow 0 \rightarrow \mathcal{D} \cdot \mathbb{I}_{b_{n}} \rightarrow \mathcal{D} \cdot s^{-1} \mathbb{I}_{b_{n}} \rightarrow 0 \rightarrow \cdots \rightarrow 0 .
$$

Since

$$
P_{n}(B)=\bigoplus_{b_{n+1} \in B_{n+1}} \mathcal{D} \cdot s^{-1} \mathbb{I}_{b_{n+1}} \oplus \bigoplus_{b_{n} \in B_{n}} \mathcal{D} \cdot \mathbb{I}_{b_{n}}(n>0) \quad \text { and } \quad P_{0}(B)=\bigoplus_{b_{1} \in B_{1}} \mathcal{D} \cdot s^{-1} \mathbb{I}_{b_{1}}
$$

the free non-negatively graded $\mathcal{D}$-module $P(B)$ is projective in each degree, what justifies the chosen notation. On the other hand, the differential $d_{P}$ of $P(B)$ is the degree -1 square 0 $\mathcal{D}$-linear map induced by the differentials in the $n$-discs and thus defined on $P_{n}(B)$ by

$$
d_{P}\left(s^{-1} \mathbb{I}_{b_{n+1}}\right)=0 \in P_{n-1}(B) \quad \text { and } \quad d_{P}\left(\mathbb{I}_{b_{n}}\right)=s^{-1} \mathbb{I}_{b_{n}} \in P_{n-1}(B)
$$

(see Lemma 16). The canonical projection $\varepsilon: P(B) \rightarrow B$, is defined on $P_{n}(B)$, as degree 0 $\mathcal{D}$-linear map, by

$$
\varepsilon\left(s^{-1} \mathbb{I}_{b_{n+1}}\right)=d_{B}\left(b_{n+1}\right) \in B_{n} \quad \text { and } \quad \varepsilon\left(\mathbb{I}_{b_{n}}\right)=b_{n} \in B_{n} .
$$

It is clearly a DGDM-morphism and extends to a DGDA-morphism $\varepsilon: \mathcal{S}(P(B)) \rightarrow B$ (see Lemma 17).

We define now the aforementioned DGDA-morphisms $i: A \rightarrow A \otimes \mathcal{S}(P(B))$ and $p: A \otimes$ $\mathcal{S}(P(B)) \rightarrow B$, where $i$ is a weak equivalence and $p$ a fibration such that $p \circ i=\phi$. We set $i=\mathrm{id}_{A} \otimes 1$ and $p=\mu_{B} \circ(\phi \otimes \varepsilon)$. It is readily checked that $i$ and $p$ are DGDA-morphisms (see Proposition 11) with composite $p \circ i=\phi$. Moreover, by definition, $p$ is a fibration in DGDA, if it is surjective in degrees $n>0$ - what immediately follows from the fact that $\varepsilon$ is surjective in these degrees.

It thus suffices to show that $i$ is a weak equivalence in DGDA, i.e., that

$$
H(i): H(A) \ni[a] \rightarrow[a \otimes 1] \in H(A \otimes \mathcal{S}(P(B)))
$$


is an isomorphism of graded $\mathcal{D}$-modules. Since $\tilde{\imath}: A \rightarrow A \otimes \mathcal{O}$ is an isomorphism in DGDM, it induces an isomorphism

$$
H(\tilde{\imath}): H(A) \ni[a] \rightarrow[a \otimes 1] \in H(A \otimes \mathcal{O}) .
$$

In view of the graded $\mathcal{D}$-module isomorphism

$$
H(A \otimes \mathcal{S}(P(B))) \simeq H(A \otimes \mathcal{O}) \oplus H\left(A \otimes \mathcal{S}^{* \geq 1}(P(B))\right)
$$

we just have to prove that

$$
H\left(A \otimes \mathcal{S}^{k \geq 1}(P(B))\right)=0
$$

as graded $\mathcal{D}$-module, or, equivalently, as graded $\mathcal{O}$-module.

To that end, note that

$$
0 \longrightarrow \operatorname{ker}^{k} \mathfrak{S} \stackrel{\iota}{\longrightarrow} P(B)^{\otimes k} \stackrel{\mathfrak{S}}{\longrightarrow}\left(P(B)^{\otimes k}\right)^{\mathbb{S}_{k}} \longrightarrow 0
$$

where $k \geq 1$ and where $\mathfrak{S}$ is the averaging map, is a short exact sequence in the abelian category DGOM of differential non-negatively graded $\mathcal{O}$-modules (see Appendix 11.5, in particular Equation (94)). Since it is canonically split by the injection

$$
\mathfrak{I}:\left(P(B)^{\otimes k}\right)^{\mathbb{S}_{k}} \rightarrow P(B)^{\otimes k},
$$

and

$$
\left(P(B)^{\otimes k}\right)^{\mathbb{S}_{k}} \simeq \mathcal{S}^{k}(P(B))
$$

as DG $\mathcal{O}$-modules (see Equation (96)), we get

$$
P(B)^{\otimes k} \simeq \mathcal{S}^{k}(P(B)) \oplus \operatorname{ker}^{k} \mathfrak{S} \quad \text { and } \quad A \otimes P(B)^{\otimes k} \simeq A \otimes \mathcal{S}^{k}(P(B)) \oplus A \otimes \operatorname{ker}^{k} \mathfrak{S},
$$

as DG $\mathcal{O}$-modules. Therefore, it suffices to show that the LHS is an acyclic chain complex of $\mathcal{O}$-modules.

We begin showing that $\mathcal{D}=\mathcal{D}_{X}(X)$, where $X$ is a smooth affine algebraic variety, is a flat module over $\mathcal{O}=\mathcal{O}_{X}(X)$. Note first that, the equivalence (8)

$$
\Gamma(X, \bullet): \operatorname{qcMod}\left(\mathcal{O}_{X}\right) \rightleftarrows \operatorname{Mod}(\mathcal{O}):
$$

is exact and strong monoidal (see remark below Equation (8) ). Second, observe that $\mathcal{D}_{X}$ is a locally free $\mathcal{O}_{X}$-module, hence, a flat (and quasi-coherent) sheaf of $\mathcal{O}_{X}$-modules, i.e., $\mathcal{D}_{X} \otimes_{\mathcal{O}_{X}}$ is exact in $\operatorname{Mod}\left(\mathcal{O}_{X}\right)$. To show that $\mathcal{D} \otimes_{\mathcal{O}} \bullet$ is exact in $\operatorname{Mod}(\mathcal{O})$, consider an exact sequence

$$
0 \rightarrow M^{\prime} \rightarrow M \rightarrow M^{\prime \prime} \rightarrow 0
$$

in $\operatorname{Mod}(\mathcal{O})$. From what has been said it follows that

$$
0 \rightarrow \mathcal{D}_{X} \otimes_{\mathcal{O}_{X}} \widetilde{M^{\prime}} \rightarrow \mathcal{D}_{X} \otimes_{\mathcal{O}_{X}} \widetilde{M} \rightarrow \mathcal{D}_{X} \otimes_{\mathcal{O}_{X}} \widetilde{M^{\prime \prime}} \rightarrow 0
$$


is an exact sequence in $\operatorname{Mod}\left(\mathcal{O}_{X}\right)$, as well as an exact sequence in $\operatorname{qcMod}\left(\mathcal{O}_{X}\right)$ (kernels and cokernels of morphisms of quasi-coherent modules are known to be quasi-coherent). When applying the exact and strong monoidal global section functor, we see that

$$
0 \rightarrow \mathcal{D} \otimes_{\mathcal{O}} M^{\prime} \rightarrow \mathcal{D} \otimes_{\mathcal{O}} M \rightarrow \mathcal{D} \otimes_{\mathcal{O}} M^{\prime \prime} \rightarrow 0
$$

is exact in $\operatorname{Mod}(\mathcal{O})$.

Next, observe that

$$
H\left(A \otimes P(B)^{\otimes k}\right)=\bigoplus_{n>0} \bigoplus_{b_{n} \in B_{n}} H\left(D_{\bullet}^{n} \otimes A \otimes P(B)^{\otimes(k-1)}\right) .
$$

To prove that each of the summands of the RHS vanishes, we apply Künneth's Theorem Wei93, Theorem 3.6.3] to the complexes $D_{\bullet}^{n}$ and $A \otimes P(B)^{\otimes(k-1)}$, noticing that both, the $n$-disc $D_{\bullet}^{n}$ (which vanishes, except in degrees $n, n-1$, where it coincides with $\mathcal{D}$ ) and its boundary $d\left(D_{\bullet}^{n}\right)$ (which vanishes, except in degree $n-1$, where it coincides with $\mathcal{D}$ ), are termwise flat $\mathcal{O}$-modules. We thus get, for any $m$, a short exact sequence

$$
\begin{aligned}
0 \rightarrow \bigoplus_{p+q=m} H_{p}\left(D_{\bullet}^{n}\right) \otimes H_{q}\left(A \otimes P(B)^{\otimes(k-1)}\right) & \rightarrow H_{m}\left(D_{\bullet}^{n} \otimes A \otimes P(B)^{\otimes(k-1)}\right) \rightarrow \\
& \bigoplus_{p+q=m-1} \operatorname{Tor}_{1}\left(H_{p}\left(D_{\bullet}^{n}\right), H_{q}\left(A \otimes P(B)^{\otimes(k-1)}\right)\right) \rightarrow 0 .
\end{aligned}
$$

Finally, since $D_{\bullet}^{n}$ is acyclic, the central term of this exact sequence vanishes, since both, the first and the third, do.

To completely finish checking the requirements of Lemma 19 and thus of Theorem 18, we still have to prove that the factorization $(i, p)=(i(\phi), p(\phi))$ of $\phi$ is functorial. In other words, we must show that, for any commutative DGDA-square

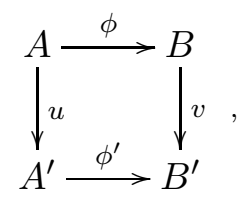

there is a commutative DGDA-diagram

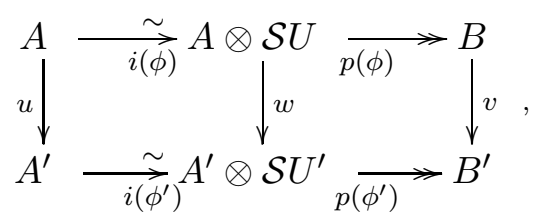

where we wrote $U$ (resp., $U^{\prime}$ ) instead of $P(B)$ (resp., $P\left(B^{\prime}\right)$ ).

To construct the DGDA-morphism $w$, we first define a DGDA-morphism $\tilde{v}: \mathcal{S} U \rightarrow \mathcal{S} U^{\prime}$, then we obtain the DGDA-morphism $w$ by setting $w=u \otimes \tilde{v}$. 
To get the DGDA-morphism $\tilde{v}$, it suffices, in view of Lemma 17, to define a degree 0 Setmap $\tilde{v}$ on $G:=\left\{s^{-1} \mathbb{I}_{b_{n}}, \mathbb{I}_{b_{n}}: b_{n} \in B_{n}, n>0\right\}$, with values in the differential graded $\mathcal{D}$-algebra $\left(\mathcal{S} U^{\prime}, d_{U^{\prime}}\right)$, which satisfies $d_{U^{\prime}} \tilde{v}=\tilde{v} d_{U}$ on $G$. We set

$$
\tilde{v}\left(s^{-1} \mathbb{I}_{b_{n}}\right)=s^{-1} \mathbb{I}_{v\left(b_{n}\right)} \in \mathcal{S} U^{\prime} \text { and } \tilde{v}\left(\mathbb{I}_{b_{n}}\right)=\mathbb{I}_{v\left(b_{n}\right)} \in \mathcal{S} U^{\prime},
$$

and easily see that all the required properties hold.

We still have to verify that the diagram (27) actually commutes. Commutativity of the left square is obvious. As for the right square, let $t:=a \otimes x_{1} \odot \ldots \odot x_{k} \in A \otimes \mathcal{S} U$, where the $x_{i}$ are elements of $U$, and note that

$$
v p(\phi)(t)=v\left(\mu_{B} \circ(\phi \otimes \varepsilon)\right)(t)=v \phi(a) \star v \varepsilon\left(x_{1}\right) \star \ldots \star v \varepsilon\left(x_{k}\right)
$$

and

$$
\begin{aligned}
p\left(\phi^{\prime}\right) w(t) & =\left(\mu_{B^{\prime}} \circ\left(\phi^{\prime} \otimes \varepsilon^{\prime}\right)\right)\left(u(a) \otimes \tilde{v}\left(x_{1}\right) \odot \ldots \odot \tilde{v}\left(x_{k}\right)\right) \\
& =\phi^{\prime} u(a) \star \varepsilon^{\prime} \tilde{v}\left(x_{1}\right) \star \ldots \star \varepsilon^{\prime} \tilde{v}\left(x_{k}\right),
\end{aligned}
$$

where $\star$ denotes the multiplication in $B^{\prime}$. Since the square (26) commutes, it suffices to check that

$$
v \varepsilon(x)=\varepsilon^{\prime} \tilde{v}(x),
$$

for any $x \in U$. However, the $\mathcal{D}$-module $U$ is freely generated by $G$ and the four involved morphisms are $\mathcal{D}$-linear: it is enough that (28) holds on $G$ - what is actually the case.

\subsection{Transferred model structure}

We proved in Theorem 12 that DGDM is a finitely generated model category whose set of generating cofibrations (resp., trivial cofibrations) is

$$
I=\left\{\iota_{k}: S_{\bullet}^{k-1} \rightarrow D_{\bullet}^{k}, k \geq 0\right\}
$$

(resp.,

$$
\left.J=\left\{\zeta_{k}: 0 \rightarrow D_{\bullet}^{k}, k \geq 1\right\}\right) .
$$

Theorem 18 thus allows us to conclude that:

Theorem 22. The category DGDA of differential non-negatively graded commutative $\mathcal{D}$ algebras is a finitely (and thus a cofibrantly) generated model category (in the sense of [GS06] and in the sense of [Hov07]), with $\mathcal{S} I=\left\{\mathcal{S}_{\iota_{k}}: \iota_{k} \in I\right\}$ as its generating set of cofibrations and $\mathcal{S} J=\left\{\mathcal{S} \zeta_{k}: \zeta_{k} \in J\right\}$ as its generating set of trivial cofibrations. The weak equivalences are the DGDA-morphisms that induce an isomorphism in homology. The fibrations are the DGDA-morphisms that are surjective in all positive degrees $p>0$.

The cofibrations will be described below.

Quillen's transfer principle actually provides a GS06-cofibrantly-generated (hence, a [Hov07]-cofibrantly-generated) [GS06-model structure on DGDA (hence, a [Hov07]-model structure, if we choose for instance the functorial factorizations given by the small object argument). 
In fact, this model structure is finitely generated, i.e. the domains and codomains of the maps in $\mathcal{S} I$ and $\mathcal{S} J$ are $n$-small DGDA-objects, $n \in \mathbb{N}$, relative to Cof. Indeed, these sources and targets are $\mathcal{S} D_{\bullet}^{k}(k \geq 1), \mathcal{S} S_{\bullet}^{k}(k \geq 0)$, and $\mathcal{O}$. We already observed (see Theorem 12) that $D_{\bullet}^{k}(k \geq 1), S_{\bullet}^{k}(k \geq 0)$, and 0 are $n$-small DGDM-objects with respect to all DGDM-morphisms.

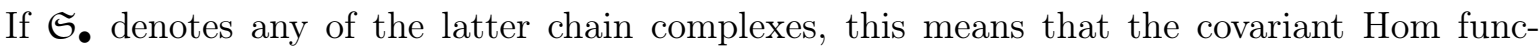
tor $\operatorname{Hom}_{\mathrm{DGDM}}\left(\mathfrak{S}_{\bullet},-\right)$ commutes with all DGDM-colimits $\operatorname{colim}_{\beta<\lambda} M_{\beta}$ • for all limit ordinals $\lambda$. It therefore follows from the adjointness property (20) and the equation (24) that, for any DGDA-colimit $\operatorname{colim}_{\beta<\lambda} A_{\beta, \bullet}$, we have

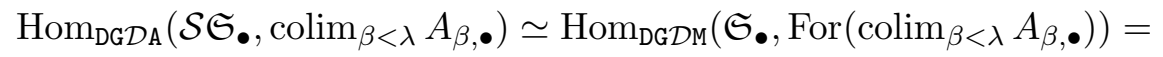

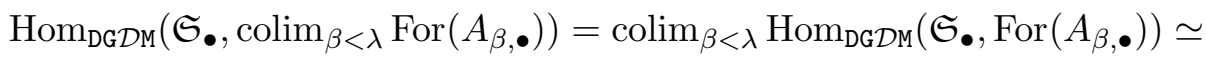

$$
\begin{aligned}
& \operatorname{colim}_{\beta<\lambda} \operatorname{Hom}_{\mathrm{DGDA}}\left(\mathcal{S S}_{\bullet}, A_{\beta, \bullet}\right) \text {. }
\end{aligned}
$$

\section{Description of DGDA-cofibrations}

\subsection{Preliminaries}

The next lemma allows us to define non-split RSDA-s, as well as DGDA-morphisms from such an RSDA into another differential graded $\mathcal{D}$-algebra.

Lemma 23. Let $\left(T, d_{T}\right) \in \mathrm{DGDA}$, let $\left(g_{j}\right)_{j \in J}$ be a family of symbols of degree $n_{j} \in \mathbb{N}$, and let $V=\bigoplus_{j \in J} \mathcal{D} \cdot g_{j}$ be the free non-negatively graded $\mathcal{D}$-module with homogeneous basis $\left(g_{j}\right)_{j \in J}$.

(i) To endow the graded $\mathcal{D}$-algebra $T \otimes \mathcal{S} V$ with a differential graded $\mathcal{D}$-algebra structure d, it suffices to define

$$
d g_{j} \in T_{n_{j}-1} \cap d_{T}^{-1}\{0\},
$$

to extend $d$ as $\mathcal{D}$-linear map to $V$, and to equip $T \otimes \mathcal{S} V$ with the differential d given, for any $t \in T_{p}, v_{1} \in V_{n_{1}}, \ldots, v_{k} \in V_{n_{k}}$, by

$$
\begin{gathered}
d\left(t \otimes v_{1} \odot \ldots \odot v_{k}\right)= \\
d_{T}(t) \otimes v_{1} \odot \ldots \odot v_{k}+(-1)^{p} \sum_{\ell=1}^{k}(-1)^{n_{\ell} \sum_{j<\ell} n_{j}}\left(t * d\left(v_{\ell}\right)\right) \otimes v_{1} \odot \ldots \widehat{\ell} \ldots \odot v_{k},
\end{gathered}
$$

where $*$ is the multiplication in $T$. If $J$ is a well-ordered set, the natural map

$$
\left(T, d_{T}\right) \ni t \mapsto t \otimes 1_{\mathcal{O}} \in(T \otimes \mathcal{S} V, d)
$$

is a RSDA.

(ii) Moreover, if $\left(B, d_{B}\right) \in \mathrm{DGDA}$ and $p \in \mathrm{DGDA}(T, B)$, it suffices - to define a morphism $q \in \mathrm{DGDA}(T \otimes \mathcal{S} V, B)$ (where the differential graded $\mathcal{D}$-algebra $(T \otimes \mathcal{S} V, d)$ is constructed as described in (i)) - to define

$$
q\left(g_{j}\right) \in B_{n_{j}} \cap d_{B}^{-1}\left\{p d\left(g_{j}\right)\right\}
$$


to extend $q$ as $\mathcal{D}$-linear map to $V$, and to define $q$ on $T \otimes \mathcal{S} V$ by

$$
q\left(t \otimes v_{1} \odot \ldots \odot v_{k}\right)=p(t) \star q\left(v_{1}\right) \star \ldots \star q\left(v_{k}\right),
$$

where $\star$ denotes the multiplication in $B$.

The reader might consider that the definition of $d(t \otimes f), f \in \mathcal{O}$, is not an edge case of Equation (23); if so, it suffices to add the definition $d(t \otimes f)=d_{T}(t) \otimes f$. Note also that Equation (23) is the only possible one. Indeed, denote the multiplication in $T \otimes \mathcal{S} V$ (see Equation (13)) by $\diamond$ and choose, to simplify, $k=2$. Then, if $d$ is any differential, which is compatible with the graded $\mathcal{D}$-algebra structure of $T \otimes \mathcal{S} V$, and which coincides with $d_{T}(t) \otimes 1_{\mathcal{O}} \simeq d_{T}(t)$ on any $t \otimes 1_{\mathcal{O}} \simeq t \in T$ (since $\left(T, d_{T}\right) \rightarrow(T \otimes \mathcal{S} V, d)$ must be a DGDAmorphism) and with $d(v) \otimes 1_{\mathcal{O}} \simeq d(v)$ on any $1_{T} \otimes v \simeq v \in V$ (since $d(v) \in T$ ), then we have necessarily

$$
\begin{gathered}
d\left(t \otimes v_{1} \odot v_{2}\right)= \\
d\left(t \otimes 1_{\mathcal{O}}\right) \diamond\left(1_{T} \otimes v_{1}\right) \diamond\left(1_{T} \otimes v_{2}\right)+ \\
(-1)^{p}\left(t \otimes 1_{\mathcal{O}}\right) \diamond d\left(1_{T} \otimes v_{1}\right) \diamond\left(1_{T} \otimes v_{2}\right)+ \\
(-1)^{p+n_{1}}\left(t \otimes 1_{\mathcal{O}}\right) \diamond\left(1_{T} \otimes v_{1}\right) \diamond d\left(1_{T} \otimes v_{2}\right)= \\
\left(d_{T}(t) \otimes 1_{\mathcal{O}}\right) \diamond\left(1_{T} \otimes v_{1}\right) \diamond\left(1_{T} \otimes v_{2}\right)+ \\
(-1)^{p}\left(t \otimes 1_{\mathcal{O}}\right) \diamond\left(d\left(v_{1}\right) \otimes 1_{\mathcal{O}}\right) \diamond\left(1_{T} \otimes v_{2}\right)+ \\
(-1)^{p+n_{1}}\left(t \otimes 1_{\mathcal{O}}\right) \diamond\left(1_{T} \otimes v_{1}\right) \diamond\left(d\left(v_{2}\right) \otimes 1_{\mathcal{O}}\right)= \\
d_{T}(t) \otimes v_{1} \odot v_{2}+(-1)^{p}\left(t * d\left(v_{1}\right)\right) \otimes v_{2}+(-1)^{p+n_{1} n_{2}}\left(t * d\left(v_{2}\right)\right) \otimes v_{1} .
\end{gathered}
$$

An analogous remark holds for Equation (34).

Proof. It is easily checked that the RHS of Equation (23) is graded symmetric in its arguments $v_{i}$ and $\mathcal{O}$-linear with respect to all arguments. Hence, the map $d$ is a degree $-1 \mathcal{O}$-linear map that is well-defined on $T \otimes \mathcal{S} V$. To show that $d$ endows $T \otimes \mathcal{S} V$ with a differential graded $\mathcal{D}$-algebra structure, it remains to prove that $d$ squares to 0 , is $\mathcal{D}$-linear and is a graded derivation for $\diamond$. The last requirement follows immediately from the definition, for $\mathcal{D}$-linearity it suffices to prove linearity with respect to the action of vector fields - what is a straightforward verification, whereas 2-nilpotency is a consequence of Condition (31). The proof of (ii) is similar.

We are now prepared to give an example of a non-split RSDA. 
Example 24. Consider the generating cofibrations $\iota_{n}: S^{n-1} \rightarrow D^{n}, n \geq 1$, and $\iota_{0}: 0 \rightarrow S^{0}$ of the model structure of DGDM. The pushouts of the induced generating cofibrations

$$
\psi_{n}=\mathcal{S}\left(\iota_{n}\right) \quad \text { and } \quad \psi_{0}=\mathcal{S}\left(\iota_{0}\right)
$$

of the transferred model structure on DGDA are important instances of RSDA-s - see Figure 2 and Equations (35), (8.1), (37), (39), and (40).

Proof. We first consider a pushout diagram for $\psi:=\psi_{n}$, for $n \geq 1$ : see Figure 1, where

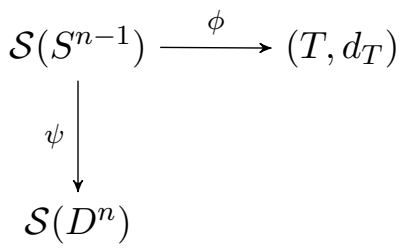

Figure 1: Pushout diagram

$\left(T, d_{T}\right) \in \mathrm{DGDA}$ and where $\phi:\left(\mathcal{S}\left(S^{n-1}\right), 0\right) \rightarrow\left(T, d_{T}\right)$ is a DGDA-morphism.

In the following, the generator of $S^{n-1}$ (resp., the generators of $D^{n}$ ) will be denoted by $1_{n-1}$ (resp., by $\mathbb{I}_{n}$ and $s^{-1} \mathbb{I}_{n}$, where $s^{-1}$ is the desuspension operator).

Note that, since $\mathcal{S}\left(S^{n-1}\right)$ is the free DGDA over the DGDM $S^{n-1}$, the DGDA-morphism $\phi$ is uniquely defined by the DGDM-morphism $\left.\phi\right|_{S^{n-1}}: S^{n-1} \rightarrow \operatorname{For}\left(T, d_{T}\right)$, where For is the forgetful functor. Similarly, since $S^{n-1}$ is, as $G \mathcal{D} M$, free over its generator $1_{n-1}$, the restriction $\left.\phi\right|_{S^{n-1}}$ is, as GDM-morphism, completely defined by its value $\phi\left(1_{n-1}\right) \in T_{n-1}$. The map $\left.\phi\right|_{S^{n-1}}$ is then a DGDM-morphism if and only if we choose

$$
\kappa_{n-1}:=\phi\left(1_{n-1}\right) \in \operatorname{ker}_{n-1} d_{T} .
$$

We now define the pushout of $(\psi, \phi)$ : see Figure 2, In the latter diagram, the differential

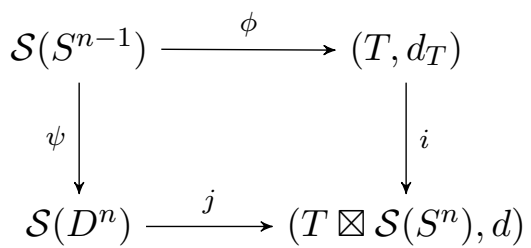

Figure 2: Completed pushout diagram

$d$ of the GDA $T \otimes \mathcal{S}\left(S^{n}\right)$ is defined as described in Lemma 23. Indeed, we deal here with the free non-negatively graded $\mathcal{D}$-module $S^{n}=S_{n}^{n}=\mathcal{D} \cdot 1_{n}$ and set

$$
d\left(1_{n}\right):=\kappa_{n-1}=\phi\left(1_{n-1}\right) \in \operatorname{ker}_{n-1} d_{T} .
$$


Hence, if $x_{\ell} \cdot 1_{n} \in \mathcal{D} \cdot 1_{n}$ (to simplify notation we denote in the following by $x_{\ell}$ both, the differential operator $x_{\ell} \in \mathcal{D}$ and the element $\left.x_{\ell} \cdot 1_{n} \in S^{n}\right)$, we get $d\left(x_{\ell}\right)=x_{\ell} \cdot \kappa_{n-1}$, and, if $t \in T_{p}$, we obtain

$$
\begin{gathered}
d\left(t \otimes x_{1} \odot \ldots \odot x_{k}\right)= \\
d_{T}(t) \otimes x_{1} \odot \ldots \odot x_{k}+(-1)^{p} \sum_{\ell=1}^{k}(-1)^{n(\ell-1)}\left(t *\left(x_{\ell} \cdot \kappa_{n-1}\right)\right) \otimes x_{1} \odot \ldots \widehat{\ell} \ldots \odot x_{k},
\end{gathered}
$$

see Equation (23). Finally the map

$$
i:\left(T, d_{T}\right) \ni t \mapsto t \otimes 1_{\mathcal{O}} \in\left(T \otimes \mathcal{S}\left(S^{n}\right), d\right)
$$

is a (non-split) RSDA, see Definition 15 .

Just as $\phi$, the DGDA-morphism $j$ is completely defined if we define it as DGDM-morphism on $D^{n}$. The choices of $j\left(\mathbb{I}_{n}\right)$ and $j\left(s^{-1} \mathbb{I}_{n}\right)$ define $j$ as GDM-morphism. The commutation condition of $j$ with the differentials reads

$$
j\left(s^{-1} \mathbb{I}_{n}\right)=d j\left(\mathbb{I}_{n}\right):
$$

only $j\left(\mathbb{I}_{n}\right)$ can be chosen freely in $\left(T \otimes \mathcal{S}\left(S^{n}\right)\right)_{n}$.

The diagram of Figure 2 is now fully described. To show that it commutes, observe that, since the involved maps $\phi, i, \psi$, and $j$ are all DGDA-morphisms, it suffices to check commutation for the arguments $1_{\mathcal{O}}$ and $1_{n-1}$. Since differential graded $\mathcal{D}$-algebras are systematically assumed to be unital, only the second case is non-obvious. We get the condition

$$
d j\left(\mathbb{I}_{n}\right)=\kappa_{n-1} \otimes 1_{\mathcal{O}} .
$$

It now suffices to set

$$
j\left(\mathbb{I}_{n}\right)=1_{T} \otimes 1_{n} \in\left(T \otimes \mathcal{S}\left(S^{n}\right)\right)_{n} .
$$

To prove that the commuting diagram of Figure 2 is the searched for pushout, it now suffices to prove its universality. Therefore, take $\left(B, d_{B}\right) \in \mathrm{DGDA}$, as well as two DGDAmorphisms $i^{\prime}:\left(T, d_{T}\right) \rightarrow\left(B, d_{B}\right)$ and $j^{\prime}: \mathcal{S}\left(D^{n}\right) \rightarrow\left(B, d_{B}\right)$, such that $j^{\prime} \circ \psi=i^{\prime} \circ \phi$, and show that there is a unique DGDA-morphism $\chi:\left(T \otimes \mathcal{S}\left(S^{n}\right), d\right) \rightarrow\left(B, d_{B}\right)$, such that $\chi \circ i=i^{\prime}$ and $\chi \circ j=j^{\prime}$.

If $\chi$ exists, we have necessarily

$$
\begin{gathered}
\chi\left(t \otimes x_{1} \odot \ldots \odot x_{k}\right)=\chi\left(\left(t \otimes 1_{\mathcal{O}}\right) \diamond\left(1_{T} \otimes x_{1}\right) \diamond \ldots \diamond\left(1_{T} \otimes x_{k}\right)\right) \\
=\chi(i(t)) \star \chi\left(1_{T} \otimes x_{1}\right) \star \ldots \star \chi\left(1_{T} \otimes x_{k}\right),
\end{gathered}
$$

where we used the same notation as above. Since any differential operator is generated by functions and vector fields, we get

$$
\chi\left(1_{T} \otimes x_{i}\right)=\chi\left(1_{T} \otimes x_{i} \cdot 1_{n}\right)=x_{i} \cdot \chi\left(1_{T} \otimes 1_{n}\right)=x_{i} \cdot \chi\left(j\left(\mathbb{I}_{n}\right)\right)=x_{i} \cdot j^{\prime}\left(\mathbb{I}_{n}\right)=j^{\prime}\left(x_{i} \cdot \mathbb{I}_{n}\right) .
$$


When combining (41) and (42), we see that, if $\chi$ exists, it is necessarily defined by

$$
\chi\left(t \otimes x_{1} \odot \ldots \odot x_{k}\right)=i^{\prime}(t) \star j^{\prime}\left(x_{1} \cdot \mathbb{I}_{n}\right) \star \ldots \star j^{\prime}\left(x_{k} \cdot \mathbb{I}_{n}\right) .
$$

This solves the question of uniqueness.

We now convince ourselves that (43) defines a DGDA-morphism $\chi$ (let us mention explicitly that we set in particular $\chi(t \otimes f)=f \cdot i^{\prime}(t)$, if $\left.f \in \mathcal{O}\right)$. It is straightforwardly verified that $\chi$ is a well-defined $\mathcal{D}$-linear map of degree 0 from $T \otimes \mathcal{S}\left(S^{n}\right)$ to $B$, which respects the multiplications and the units. The interesting point is the chain map property of $\chi$. Indeed, consider, to simplify, the argument $t \otimes x$, what will disclose all relevant insights. Assume again that $t \in T_{p}$ and $x \in S^{n}$, and denote the differential of $\mathcal{S}\left(D^{n}\right)$, just as its restriction to $D^{n}$, by $s^{-1}$. It follows that

$$
d_{B}(\chi(t \otimes x))=i^{\prime}\left(d_{T}(t)\right) \star j^{\prime}\left(x \cdot \mathbb{I}_{n}\right)+(-1)^{p} i^{\prime}(t) \star j^{\prime}\left(x \cdot s^{-1} \mathbb{I}_{n}\right) .
$$

Since $\psi\left(1_{n-1}\right)=s^{-1} \mathbb{I}_{n}$ and $j^{\prime} \circ \psi=i^{\prime} \circ \phi$, we obtain $j^{\prime}\left(s^{-1} \mathbb{I}_{n}\right)=i^{\prime}\left(\phi\left(1_{n-1}\right)\right)=i^{\prime}\left(\kappa_{n-1}\right)$. Hence,

$$
\begin{gathered}
d_{B}(\chi(t \otimes x))=\chi\left(d_{T}(t) \otimes x\right)+(-1)^{p} i^{\prime}(t) \star i^{\prime}\left(x \cdot \kappa_{n-1}\right)= \\
\chi\left(d_{T}(t) \otimes x+(-1)^{p} t *\left(x \cdot \kappa_{n-1}\right)\right)=\chi(d(t \otimes x)) .
\end{gathered}
$$

As afore-mentioned, no new feature appears, if we replace $t \otimes x$ by a general argument.

As the conditions $\chi \circ i=i^{\prime}$ and $\chi \circ j=j^{\prime}$ are easily checked, this completes the proof of the statement that any pushout of any $\psi_{n}, n \geq 1$, is a $\operatorname{RSDA}$.

The proof of the similar claim for $\psi_{0}$ is analogous and even simpler, and will not be detailed here.

Actually pushouts of $\psi_{0}$ are border cases of pushouts of the $\psi_{n}$-s, $n \geq 1$. In other words, to obtain a pushout of $\psi_{0}$, it suffices to set, in Figure 2 and in Equation (8.1), the degree $n$ to 0 . Since we consider exclusively non-negatively graded complexes, we then get $\mathcal{S}\left(S^{-1}\right)=$ $\mathcal{S}(0)=\mathcal{O}, \mathcal{S}\left(D^{0}\right)=\mathcal{S}\left(S^{0}\right)$, and $\kappa_{-1}=0$.

\subsection{DGDA-cofibrations}

The following theorem characterizes the cofibrations of the cofibrantly generated model structure we constructed on DGDA.

Theorem 25. The DGDA-cofibrations are exactly the retracts of the relative Sullivan $\mathcal{D}$ algebras.

Since the DGDA-cofibrations are exactly the retracts of the transfinite compositions of pushouts of generating cofibrations

$$
\psi_{n}: \mathcal{S}\left(S^{n-1}\right) \rightarrow \mathcal{S}\left(D^{n}\right), \quad n \geq 0
$$

the proof of Theorem 25 reduces to the proof of 
Theorem 26. The transfinite compositions of pushouts of $\psi_{n}-s, n \geq 0$, are exactly the relative Sullivan $\mathcal{D}$-algebras.

Lemma 27. For any $M, N \in \mathrm{DGDM}$, we have

$$
\mathcal{S}(M \oplus N) \simeq \mathcal{S} M \otimes \mathcal{S} N
$$

in DGDA .

Proof. It suffices to remember that the binary coproduct in the category $\mathrm{DGDM}=\mathrm{Ch}_{+}(\mathcal{D})$ (resp., the category DGDA $=\mathrm{CMon}(\mathrm{DGDM})$ ) of non-negatively graded chain complexes of $\mathcal{D}$ modules (resp., of commutative monoids in DGDM) is the direct sum (resp., the tensor product). The conclusion then follows from the facts that $\mathcal{S}$ is the left adjoint of the forgetful functor and that any left adjoint commutes with colimits.

Any ordinal is zero, a successor ordinal, or a limit ordinal. We denote the class of all successor ordinals (resp., all limit ordinals) by $\mathfrak{O}_{s}$ (resp., $\mathfrak{O}_{\ell}$ ).

Proof of Theorem [26. (i) Consider an ordinal $\lambda$ and a $\lambda$-sequence in DGDA, i.e., a colimit respecting functor $X: \lambda \rightarrow \mathrm{DGDA}$ (here $\lambda$ is viewed as the category whose objects are the ordinals $\alpha<\lambda$ and which contains a unique morphism $\alpha \rightarrow \beta$ if and only if $\alpha \leq \beta$ ):

$$
X_{0} \rightarrow X_{1} \rightarrow \ldots \rightarrow X_{n} \rightarrow X_{n+1} \rightarrow \ldots X_{\omega} \rightarrow X_{\omega+1} \rightarrow \ldots \rightarrow X_{\alpha} \rightarrow X_{\alpha+1} \rightarrow \ldots
$$

We assume that, for any $\alpha$ such that $\alpha+1<\lambda$, the morphism $X_{\alpha} \rightarrow X_{\alpha+1}$ is a pushout of some $\psi_{n_{\alpha+1}}\left(n_{\alpha+1} \geq 0\right)$. Then the morphism $X_{0} \rightarrow \operatorname{colim}_{\alpha<\lambda} X_{\alpha}$ is exactly what we call a transfinite composition of pushouts of $\psi_{n}$-s. Our task is to show that this morphism is a RSDA.

We first compute the terms $X_{\alpha}, \alpha<\lambda$, of the $\lambda$-sequence, then we determine its colimit. For $\alpha<\lambda$ (resp., for $\alpha<\lambda, \alpha \in \mathfrak{O}_{s}$ ), we denote the differential graded $\mathcal{D}$-algebra $X_{\alpha}$ (resp., the DGDA-morphism $\left.X_{\alpha-1} \rightarrow X_{\alpha}\right)$ by $\left(A_{\alpha}, d_{\alpha}\right)$ (resp., by $X_{\alpha, \alpha-1}:\left(A_{\alpha-1}, d_{\alpha-1}\right) \rightarrow\left(A_{\alpha}, d_{\alpha}\right)$ ). Since $X_{\alpha, \alpha-1}$ is the pushout of some $\psi_{n_{\alpha}}$ along some DGDA-morphism $\phi_{\alpha}$, its target algebra is of the form

$$
\left(A_{\alpha}, d_{\alpha}\right)=\left(A_{\alpha-1} \otimes \mathcal{S}\left\langle a_{\alpha}\right\rangle, d_{\alpha}\right)
$$

and $X_{\alpha, \alpha-1}$ is the canonical inclusion

$$
X_{\alpha, \alpha-1}:\left(A_{\alpha-1}, d_{\alpha-1}\right) \ni \mathfrak{a}_{\alpha-1} \mapsto \mathfrak{a}_{\alpha-1} \otimes 1_{\mathcal{O}} \in\left(A_{\alpha-1} \otimes \mathcal{S}\left\langle a_{\alpha}\right\rangle, d_{\alpha}\right),
$$

see Example 24. Here $a_{\alpha}$ is the generator $1_{n_{\alpha}}$ of $S^{n_{\alpha}}$ and $\left\langle a_{\alpha}\right\rangle$ is the free non-negatively graded $\mathcal{D}$-module $S^{n_{\alpha}}=\mathcal{D} \cdot a_{\alpha}$ concentrated in degree $n_{\alpha}$; further, the differential

$$
d_{\alpha} \text { is defined by (8.1) from } d_{\alpha-1} \text { and } \kappa_{n_{\alpha}-1}:=\phi_{\alpha}\left(1_{n_{\alpha}-1}\right) \text {. }
$$

In particular, $A_{1}=A_{0} \otimes \mathcal{S}\left\langle a_{1}\right\rangle, d_{1}\left(a_{1}\right)=\kappa_{n_{1}-1}=\phi_{1}\left(1_{n_{1}-1}\right) \in A_{0}$, and $X_{10}: A_{0} \rightarrow A_{1}$ is the inclusion. 
Lemma 28. For any $\alpha<\lambda$, we have

$$
A_{\alpha} \simeq A_{0} \otimes \mathcal{S}\left\langle a_{\delta}: \delta \leq \alpha, \delta \in \mathfrak{O}_{s}\right\rangle
$$

as a graded $\mathcal{D}$-algebra, and

$$
d_{\alpha}\left(a_{\delta}\right) \in A_{0} \otimes \mathcal{S}\left\langle a_{\varepsilon}: \varepsilon<\delta, \varepsilon \in \mathfrak{O}_{s}\right\rangle
$$

for all $\delta \leq \alpha, \delta \in \mathfrak{O}_{s}$. Moreover, for any $\gamma \leq \beta \leq \alpha<\lambda$, we have

$$
A_{\beta}=A_{\gamma} \otimes \mathcal{S}\left\langle a_{\delta}: \gamma<\delta \leq \beta, \delta \in \mathfrak{O}_{s}\right\rangle
$$

and the DGDA-morphism $X_{\beta \gamma}$ is the natural inclusion

$$
X_{\beta \gamma}:\left(A_{\gamma}, d_{\gamma}\right) \ni \mathfrak{a}_{\gamma} \mapsto \mathfrak{a}_{\gamma} \otimes 1_{\mathcal{O}} \in\left(A_{\beta}, d_{\beta}\right) .
$$

Since the latter statement holds in particular for $\gamma=0$ and $\beta=\alpha$, the DGDA-inclusion $X_{\alpha 0}$ : $\left(A_{0}, d_{0}\right) \rightarrow\left(A_{\alpha}, d_{\alpha}\right)$ is a RSDA (for the natural ordering of $\left\{a_{\delta}: \delta \leq \alpha, \delta \in \mathfrak{O}_{s}\right\}$ ).

Proof of Lemma 28. To prove that this claim (i.e., Equations (47) - (49)) is valid for all ordinals that are smaller than $\lambda$, we use a transfinite induction. Since the assertion obviously holds for $\alpha=1$, it suffices to prove these properties for $\alpha<\lambda$, assuming that they are true for all $\beta<\alpha$. We distinguish (as usually in transfinite induction) the cases $\alpha \in \mathfrak{O}_{s}$ and $\alpha \in \mathfrak{O}_{\ell}$.

If $\alpha \in \mathfrak{O}_{s}$, it follows from Equation (44), from the induction assumption, and from Lemma 27, that

$$
A_{\alpha}=A_{\alpha-1} \otimes \mathcal{S}\left\langle a_{\alpha}\right\rangle \simeq A_{0} \otimes \mathcal{S}\left\langle a_{\delta}: \delta \leq \alpha, \delta \in \mathfrak{O}_{s}\right\rangle,
$$

as graded $\mathcal{D}$-algebra. Further, in view of Equation (46) and the induction hypothesis, we get

$$
d_{\alpha}\left(a_{\alpha}\right)=\phi_{\alpha}\left(1_{n_{\alpha}-1}\right) \in A_{\alpha-1}=A_{0} \otimes \mathcal{S}\left\langle a_{\delta}: \delta<\alpha, \delta \in \mathfrak{O}_{s}\right\rangle
$$

and, for $\delta \leq \alpha-1, \delta \in \mathfrak{O}_{s}$,

$$
d_{\alpha}\left(a_{\delta}\right)=d_{\alpha-1}\left(a_{\delta}\right) \in A_{0} \otimes \mathcal{S}\left\langle a_{\gamma}: \gamma<\delta, \gamma \in \mathfrak{O}_{s}\right\rangle .
$$

Finally, as concerns $X_{\beta \gamma}$, the unique case to check is $\gamma \leq \alpha-1$ and $\beta=\alpha$. The DGDA-map $X_{\alpha-1, \gamma}$ is an inclusion

$$
X_{\alpha-1, \gamma}: A_{\gamma} \ni \mathfrak{a}_{\gamma} \mapsto \mathfrak{a}_{\gamma} \otimes 1_{\mathcal{O}} \in A_{\alpha-1}
$$

(by induction), and so is the DGDA-map

$$
X_{\alpha, \alpha-1}: A_{\alpha-1} \ni \mathfrak{a}_{\alpha-1} \mapsto \mathfrak{a}_{\alpha-1} \otimes 1_{\mathcal{O}} \in A_{\alpha}
$$

(in view of (45)). The composite $X_{\alpha \gamma}$ is thus a DGDA-inclusion as well.

In the case $\alpha \in \mathfrak{O}_{\ell}$, i.e., $\alpha=\operatorname{colim}_{\beta<\alpha} \beta$, we obtain $\left(A_{\alpha}, d_{\alpha}\right)=\operatorname{colim}_{\beta<\alpha}\left(A_{\beta}, d_{\beta}\right)$ in $\mathrm{DGDA}$, since $X$ is a colimit respecting functor. The index set $\alpha$ is well-ordered, hence, it is a directed poset. Moreover, for any $\delta \leq \gamma \leq \beta<\alpha$, the DGDA-maps $X_{\beta \delta}, X_{\gamma \delta}$, and $X_{\beta \gamma}$ 
satisfy $X_{\beta \delta}=X_{\beta \gamma} \circ X_{\gamma \delta}$. It follows that the family $\left(A_{\beta}, d_{\beta}\right)_{\beta<\alpha}$, together with the family $X_{\beta \gamma}, \gamma \leq \beta<\alpha$, is a direct system in DGDA, whose morphisms are, in view of the induction assumption, natural inclusions

$$
X_{\beta \gamma}: A_{\gamma} \ni \mathfrak{a}_{\gamma} \mapsto \mathfrak{a}_{\gamma} \otimes 1_{\mathcal{O}} \in A_{\beta}
$$

The colimit $\left(A_{\alpha}, d_{\alpha}\right)=\operatorname{colim}_{\beta<\alpha}\left(A_{\beta}, d_{\beta}\right)$ is thus a direct limit. However, a direct limit in DGDA coincides with the corresponding direct limit in DGDM, or even in Set (which is then naturally endowed with a differential graded $\mathcal{D}$-algebra structure). As a set, the direct limit $\left(A_{\alpha}, d_{\alpha}\right)=\operatorname{colim}_{\beta<\alpha}\left(A_{\beta}, d_{\beta}\right)$ is given by

$$
A_{\alpha}=\coprod_{\beta<\alpha} A_{\beta} / \sim
$$

where $\sim$ means that we identify $\mathfrak{a}_{\gamma}, \gamma \leq \beta$, with

$$
\mathfrak{a}_{\gamma} \sim X_{\beta \gamma}\left(\mathfrak{a}_{\gamma}\right)=\mathfrak{a}_{\gamma} \otimes 1_{\mathcal{O}}
$$

i.e., that we identify $A_{\gamma}$ with

$$
A_{\gamma} \sim A_{\gamma} \otimes \mathcal{O} \subset A_{\beta}
$$

It follows that

$$
A_{\alpha}=\bigcup_{\beta<\alpha} A_{\beta}=A_{0} \otimes \mathcal{S}\left\langle a_{\delta}: \delta<\alpha, \delta \in \mathfrak{O}_{s}\right\rangle=A_{0} \otimes \mathcal{S}\left\langle a_{\delta}: \delta \leq \alpha, \delta \in \mathfrak{O}_{s}\right\rangle .
$$

As just mentioned, this set $A_{\alpha}$ can naturally be endowed with a differential graded $\mathcal{D}$-algebra structure. For instance, the differential $d_{\alpha}$ is defined in the obvious way from the differentials $d_{\beta}, \beta<\alpha$. In particular, any generator $a_{\delta}, \delta \leq \alpha, \delta \in \mathfrak{O}_{s}$, belongs to $A_{\delta}$. Hence, by definition of $d_{\alpha}$ and in view of the induction assumption, we get

$$
d_{\alpha}\left(a_{\delta}\right)=d_{\delta}\left(a_{\delta}\right) \in A_{0} \otimes \mathcal{S}\left\langle a_{\varepsilon}: \varepsilon<\delta, \varepsilon \in \mathfrak{O}_{s}\right\rangle .
$$

Finally, since $X$ is colimit respecting, not only $A_{\alpha}=\operatorname{colim}_{\beta<\alpha} A_{\beta}=\bigcup_{\beta<\alpha} A_{\beta}$, but, furthermore, for any $\gamma<\alpha$, the DGDA-morphism $X_{\alpha \gamma}: A_{\gamma} \rightarrow A_{\alpha}$ is the map $X_{\alpha \gamma}: A_{\gamma} \rightarrow \bigcup_{\beta<\alpha} A_{\beta}$, i.e., the canonical inclusion.

We now come back to the proof of Part (i) of Theorem 26, i.e., we now explain why the morphism $i:\left(A_{0}, d_{0}\right) \rightarrow C$, where $C=\operatorname{colim}_{\alpha<\lambda}\left(A_{\alpha}, d_{\alpha}\right)$ and where $i$ is the first of the morphisms that are part of the colimit construction, is a RSDA - see above. If $\lambda \in \mathfrak{O}_{s}$, the colimit $C$ coincides with $\left(A_{\lambda-1}, d_{\lambda-1}\right)$ and $i=X_{\lambda-1,0}$. Hence, the morphism $i$ is a RSDA in view of Lemma 28. If $\lambda \in \mathfrak{O}_{\ell}$, the colimit $C=\operatorname{colim}_{\alpha<\lambda}\left(A_{\alpha}, d_{\alpha}\right)$ is, like above, the direct limit of the direct DGDA-system $\left(X_{\alpha}=\left(A_{\alpha}, d_{\alpha}\right), X_{\alpha \beta}\right)$ indexed by the directed poset $\lambda$, whose morphisms $X_{\alpha \beta}$ are, in view of Lemma 28, canonical inclusions. Hence, $C$ is again an ordinary union:

$$
C=\bigcup_{\alpha<\lambda} A_{\alpha}=A_{0} \otimes \mathcal{S}\left\langle a_{\delta}: \delta<\lambda, \delta \in \mathfrak{O}_{s}\right\rangle
$$


where the last equality is due to Lemma 28 . We define the differential $d_{C}$ on $C$ exactly as we defined the differential $d_{\alpha}$ on the direct limit in the proof of Lemma 28. It is then straightforwardly checked that $i$ is a RSDA.

(ii) We still have to show that any $\operatorname{RSDA}\left(A_{0}, d_{0}\right) \rightarrow\left(A_{0} \otimes \mathcal{S} V, d\right)$ can be constructed as a transfinite composition of pushouts of generating cofibrations $\psi_{n}, n \geq 0$. Let $\left(a_{j}\right)_{j \in J}$ be the basis of the free non-negatively graded $\mathcal{D}$-module $V$. Since $J$ is a well-ordered set, it is orderisomorphic to a unique ordinal $\mu=\{0,1, \ldots, n, \ldots, \omega, \omega+1, \ldots\}$, whose elements can thus be utilized to label the basis vectors. However, we prefer using the following order-respecting relabelling of these vectors:

$$
a_{0} \rightsquigarrow a_{1}, a_{1} \rightsquigarrow a_{2}, \ldots, a_{n} \rightsquigarrow a_{n+1}, \ldots, a_{\omega} \rightsquigarrow a_{\omega+1}, a_{\omega+1} \rightsquigarrow a_{\omega+2}, \ldots
$$

In other words, the basis vectors of $V$ can be labelled by the successor ordinals that are strictly smaller than $\lambda:=\mu+1$ (this is true, whether $\mu \in \mathfrak{O}_{s}$, or $\mu \in \mathfrak{O}_{\ell}$ ):

$$
V=\bigoplus_{\delta<\lambda, \delta \in \mathfrak{O}_{s}} \mathcal{D} \cdot a_{\delta}
$$

For any $\alpha<\lambda$, we now set

$$
\left(A_{\alpha}, d_{\alpha}\right):=\left(A_{0} \otimes \mathcal{S}\left\langle a_{\delta}: \delta \leq \alpha, \delta \in \mathfrak{O}_{s}\right\rangle,\left.d\right|_{A_{\alpha}}\right) .
$$

It is clear that $A_{\alpha}$ is a graded $\mathcal{D}$-subalgebra of $A_{0} \otimes \mathcal{S} V$. Since $A_{\alpha}$ is generated, as an algebra, by the elements of the types $\mathfrak{a}_{0} \otimes 1_{\mathcal{O}}$ and $D \cdot\left(1_{A_{0}} \otimes a_{\delta}\right), D \in \mathcal{D}, \delta \leq \alpha, \delta \in \mathfrak{O}_{s}$, and since

$$
d\left(\mathfrak{a}_{0} \otimes 1_{\mathcal{O}}\right)=d_{0}\left(\mathfrak{a}_{0}\right) \otimes 1_{\mathcal{O}} \in A_{\alpha}
$$

and

$$
d\left(D \cdot\left(1_{A_{0}} \otimes a_{\delta}\right)\right) \in A_{0} \otimes \mathcal{S}\left\langle a_{\varepsilon}: \varepsilon<\delta, \varepsilon \in \mathfrak{O}_{s}\right\rangle \subset A_{\alpha},
$$

the derivation $d$ stabilizes $A_{\alpha}$. Hence, $\left(A_{\alpha}, d_{\alpha}\right)=\left(A_{\alpha},\left.d\right|_{A_{\alpha}}\right)$ is actually a differential graded $\mathcal{D}$-subalgebra of $\left(A_{0} \otimes \mathcal{S} V, d\right)$.

If $\beta \leq \alpha<\lambda$, the algebra $\left(A_{\beta},\left.d\right|_{A_{\beta}}\right)$ is a differential graded $\mathcal{D}$-subalgebra of $\left(A_{\alpha},\left.d\right|_{A_{\alpha}}\right)$, so that the canonical inclusion $i_{\alpha \beta}:\left(A_{\beta}, d_{\beta}\right) \rightarrow\left(A_{\alpha}, d_{\alpha}\right)$ is a DGDA-morphism. In view of the techniques used in (i), it is obvious that the functor $X=\left(A_{-}, d_{-}\right): \lambda \rightarrow$ DGDA respects colimits, and that the colimit of the whole $\lambda$-sequence (remember that $\lambda=\mu+1 \in \mathfrak{O}_{s}$ ) is the algebra $\left(A_{\mu}, d_{\mu}\right)=\left(A_{0} \otimes \mathcal{S} V, d\right)$, i.e., the original algebra.

The RSDA $\left(A_{0}, d_{0}\right) \rightarrow\left(A_{0} \otimes \mathcal{S} V, d\right)$ has thus been built as transfinite composition of canonical DGDA-inclusions $i:\left(A_{\alpha}, d_{\alpha}\right) \rightarrow\left(A_{\alpha+1}, d_{\alpha+1}\right), \alpha+1<\lambda$. Recall that

$$
A_{\alpha+1}=A_{\alpha} \otimes \mathcal{S}\left\langle a_{\alpha+1}\right\rangle \simeq A_{\alpha} \otimes \mathcal{S}\left(S^{n}\right)
$$

if we set $n:=\operatorname{deg}\left(a_{\alpha+1}\right)$. It suffices to show that $i$ is a pushout of $\psi_{n}$, see Figure 3, We will detail the case $n \geq 1$. Since all the differentials are restrictions of $d$, we have $\kappa_{n-1}:=$ $d_{\alpha+1}\left(a_{\alpha+1}\right) \in A_{\alpha} \cap \operatorname{ker}_{n-1} d_{\alpha}$, and $\phi\left(1_{n-1}\right):=\kappa_{n-1}$ defines a DGDA-morphism $\phi$, see Example 


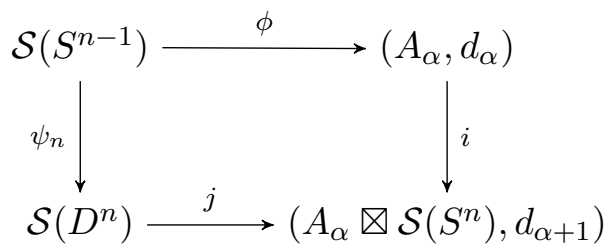

Figure 3: $i$ as pushout of $\psi_{n}$

24. When using the construction described in Example 24, we get the pushout $i:\left(A_{\alpha}, d_{\alpha}\right) \rightarrow$ $\left(A_{\alpha} \otimes \mathcal{S}\left(S^{n}\right), \partial\right)$ of $\psi_{n}$ along $\phi$. Here $i$ is the usual canonical inclusion and $\partial$ is the differential defined by Equation (8.1). It thus suffices to check that $\partial=d_{\alpha+1}$. Let $\mathfrak{a}_{\alpha} \in A_{\alpha}^{p}$ and let $x_{1} \simeq x_{1} \cdot a_{\alpha+1}, \ldots, x_{k} \simeq x_{k} \cdot a_{\alpha+1} \in \mathcal{D} \cdot a_{\alpha+1}=S^{n}$. Assume, to simplify, that $k=2$; the general case is similar. When denoting the multiplication in $A_{\alpha}$ (resp., $\left.A_{\alpha+1}=A_{\alpha} \otimes \mathcal{S}\left(S^{n}\right)\right)$ as usual by $*$ (resp., $\star)$, we obtain

$$
\begin{gathered}
\partial\left(\mathfrak{a}_{\alpha} \otimes x_{1} \odot x_{2}\right)= \\
d_{\alpha}\left(\mathfrak{a}_{\alpha}\right) \otimes x_{1} \odot x_{2}+(-1)^{p}\left(\mathfrak{a}_{\alpha} *\left(x_{1} \cdot \kappa_{n-1}\right)\right) \otimes x_{2}+(-1)^{p+n}\left(\mathfrak{a}_{\alpha} *\left(x_{2} \cdot \kappa_{n-1}\right)\right) \otimes x_{1}= \\
\left(d_{\alpha}\left(\mathfrak{a}_{\alpha}\right) \otimes 1_{\mathcal{O}}\right) \star\left(1_{A_{\alpha}} \otimes x_{1}\right) \star\left(1_{A_{\alpha}} \otimes x_{2}\right)+ \\
(-1)^{p}\left(\mathfrak{a}_{\alpha} \otimes 1_{\mathcal{O}}\right) \star\left(\left(x_{1} \cdot \kappa_{n-1}\right) \otimes 1_{\mathcal{O}}\right) \star\left(1_{A_{\alpha}} \otimes x_{2}\right)+ \\
(-1)^{p+n}\left(\mathfrak{a}_{\alpha} \otimes 1_{\mathcal{O}}\right) \star\left(1_{A_{\alpha}} \otimes x_{1}\right) \star\left(\left(x_{2} \cdot \kappa_{n-1}\right) \otimes 1_{\mathcal{O}}\right)= \\
d_{\alpha+1}\left(\mathfrak{a}_{\alpha} \otimes 1_{\mathcal{O}}\right) \star\left(1_{A_{\alpha}} \otimes x_{1}\right) \star\left(1_{A_{\alpha}} \otimes x_{2}\right)+ \\
(-1)^{p}\left(\mathfrak{a}_{\alpha} \otimes 1_{\mathcal{O}}\right) \star d_{\alpha+1}\left(1_{A_{\alpha}} \otimes x_{1}\right) \star\left(1_{A_{\alpha}} \otimes x_{1}\right)+ \\
(-1)^{p+n}\left(\mathfrak{a}_{\alpha} \otimes 1_{\mathcal{O}}\right) \star\left(1_{A_{\alpha}} \otimes x_{1}\right) \star d_{\alpha+1}\left(1_{A_{\alpha}} \otimes x_{2}\right)= \\
d_{\alpha+1}\left(\mathfrak{a}_{\alpha} \otimes x_{1} \odot x_{2}\right) .
\end{gathered}
$$

\section{$9 \quad$ Explicit functorial factorizations}

The main idea of Subsection 7.5 is the decomposition of an arbitrary DGDA-morphism $\phi: A \rightarrow B$ into a weak equivalence $i: A \rightarrow A \otimes \mathcal{S U}$ and a fibration $p: A \otimes \mathcal{S} U \rightarrow B$. It is easily seen that $i$ is a split relative Sullivan $\mathcal{D}$-algebra. Indeed,

$$
U=P(B)=\bigoplus_{n>0} \bigoplus_{b_{n} \in B_{n}} D_{\bullet}^{n} \in \mathrm{DGDM}
$$


with differential $d_{U}=d_{P}$ defined by

$$
d_{U}\left(s^{-1} \mathbb{I}_{b_{n}}\right)=0 \quad \text { and } \quad d_{U}\left(\mathbb{I}_{b_{n}}\right)=s^{-1} \mathbb{I}_{b_{n}} .
$$

Hence, $\mathcal{S} U \in \mathrm{DGD}$ A, with differential $d_{S}$ induced by $d_{U}$, and $A \otimes \mathcal{S} U \in \mathrm{DGD}$ A, with differential

$$
d_{1}=d_{A} \otimes \mathrm{id}+\mathrm{id} \otimes d_{S} .
$$

Therefore, $i: A \rightarrow A \otimes \mathcal{S} U$ is a DGDA-morphism. Since $U$ is the free non-negatively graded $\mathcal{D}$-module with homogeneous basis

$$
G=\left\{s^{-1} \mathbb{I}_{b_{n}}, \mathbb{I}_{b_{n}}: b_{n} \in B_{n}, n>0\right\},
$$

all the requirements of the definition of a split RSDA are obviously satisfied, except that we still have to check the well-ordering and the lowering condition.

Since every set can be well-ordered, we first choose a well-ordering on each $B_{n}, n>0$ : if $\lambda_{n}$ denotes the unique ordinal that belongs to the same equivalence class of well-ordered sets, the elements of $B_{n}$ can be labelled by the elements of $\lambda_{n}$. Then we define the following total order: the $s^{-1} \mathbb{I}_{b_{1}}, b_{1} \in B_{1}$, are smaller than the $\mathbb{I}_{b_{1}}$, which are smaller than the $s^{-1} \mathbb{I}_{b_{2}}$, and so on ad infinitum. The construction of an infinite decreasing sequence in this totally ordered set amounts to extracting an infinite decreasing sequence from a finite number of ordinals $\lambda_{1}, \lambda_{1}, \ldots, \lambda_{k}$. Since this is impossible, the considered total order is a well-ordering. The lowering condition is thus a direct consequence of Equations (52) and (53).

Let now $\left\{\gamma_{\alpha}: \alpha \in J\right\}$ be the set $G$ of generators endowed with the just defined well-order. Observe that, if the label $\alpha$ of the generator $\gamma_{\alpha}$ increases, its degree $\operatorname{deg} \gamma_{\alpha}$ increases as well, i.e., that

$$
\alpha \leq \beta \Rightarrow \operatorname{deg} \gamma_{\alpha} \leq \operatorname{deg} \gamma_{\beta} .
$$

Finally, any DGDA-morphism $\phi: A \rightarrow B$ admits a functorial factorization

$$
A \stackrel{i}{\longrightarrow} A \otimes \mathcal{S U} \stackrel{p}{\longrightarrow} B
$$

where $p$ is a fibration and $i$ is a weak equivalence, as well as a split RSDA. In view of Theorem 25, the morphism $i$ is thus a cofibration, with the result that we actually constructed a natural decomposition $\phi=p \circ i$ of an arbitrary DGDA-morphism $\phi$ into $i \in$ TrivCof and $p \in$ Fib. The description of this factorization is summarized below, in Theorem 29, which provides essentially an explicit natural 'Cof - TrivFib' decomposition

$$
A \stackrel{i^{\prime}}{\longrightarrow} A \otimes \mathcal{S} U^{\prime} \stackrel{p^{\prime}}{\longrightarrow} B
$$

Before stating Theorem 29, we sketch the construction of the factorization (56). To simplify, we denote algebras of the type $A \otimes \mathcal{S} V_{k}$ by $R_{V_{k}}$, or simply $R_{k}$.

We start from the 'small' 'Cof - Fib' decomposition (55) of a DGDA-morphism $A \stackrel{\phi}{\longrightarrow} B$, i.e., from the factorization $A \stackrel{i}{\longrightarrow} R_{U} \stackrel{p}{\longrightarrow} B$. To find a substitute $q$ for $p$, which is a trivial 
fibration, we mimic an idea used in the construction of the Koszul-Tate resolution: we add generators to improve homological properties.

Note first that $H(p)$ is surjective if, for any homology class $\left[\beta_{n}\right] \in H_{n}(B)$, there is a class $\left[\rho_{n}\right] \in H_{n}\left(R_{U}\right)$, such that $\left[p \rho_{n}\right]=\left[\beta_{n}\right]$. Hence, consider all the homology classes $\left[\beta_{n}\right], n \geq 0$, of $B$, choose in each class a representative $\dot{\beta}_{n} \simeq\left[\beta_{n}\right]$, and add generators $\mathbb{I}_{\dot{\beta}_{n}}$ to those of $U$. It then suffices to extend the differential $d_{1}$ (resp., the fibration $p$ ) defined on $R_{U}=A \otimes \mathcal{S} U$, so that the differential of $\mathbb{I}_{\dot{\beta}_{n}}$ vanishes (resp., so that the projection of $\mathbb{I}_{\dot{\beta}_{n}}$ coincides with $\dot{\beta}_{n}$ ) $\left(\triangleright_{1}\right.$ - this triangle is just a mark that allows us to retrieve this place later on). To get a functorial 'Cof - TrivFib' factorization, we do not add a new generator $\mathbb{I}_{\dot{\beta}_{n}}$, for each homology class $\dot{\beta}_{n} \simeq\left[\beta_{n}\right] \in H_{n}(B), n \geq 0$, but we add a new generator $\mathbb{I}_{\beta_{n}}$, for each cycle $\beta_{n} \in \operatorname{ker}_{n} d_{B}$, $n \geq 0$. Let us implement this idea in a rigorous manner. Assign the degree $n$ to $\mathbb{I}_{\beta_{n}}$ and set

$$
\begin{gathered}
V_{0}:=U \oplus G_{0}:=U \oplus\left\langle\mathbb{I}_{\beta_{n}}: \beta_{n} \in \operatorname{ker}_{n} d_{B}, n \geq 0\right\rangle= \\
\left\langle s^{-1} \mathbb{I}_{b_{n}}, \mathbb{I}_{b_{n}}, \mathbb{I}_{\beta_{n}}: b_{n} \in B_{n}, n>0, \beta_{n} \in \operatorname{ker}_{n} d_{B}, n \geq 0\right\rangle .
\end{gathered}
$$

Set now

$$
\delta_{V_{0}}\left(s^{-1} \mathbb{I}_{b_{n}}\right)=d_{1}\left(s^{-1} \mathbb{I}_{b_{n}}\right)=0, \quad \delta_{V_{0}} \mathbb{I}_{b_{n}}=d_{1} \mathbb{I}_{b_{n}}=s^{-1} \mathbb{I}_{b_{n}}, \quad \delta_{V_{0}} \mathbb{I}_{\beta_{n}}=0,
$$

thus defining, in view of Lemma 16, a differential graded $\mathcal{D}$-module structure on $V_{0}$. It follows that $\left(\mathcal{S} V_{0}, \delta_{V_{0}}\right) \in \mathrm{DGDA}$ and that

$$
\left(R_{0}, \delta_{0}\right):=\left(A \otimes \mathcal{S} V_{0}, d_{A} \otimes \mathrm{id}+\mathrm{id} \otimes \delta_{V_{0}}\right) \in \mathrm{DGDA}
$$

Similarly, we set

$$
q_{V_{0}}\left(s^{-1} \mathbb{I}_{b_{n}}\right)=p\left(s^{-1} \mathbb{I}_{b_{n}}\right)=\varepsilon\left(s^{-1} \mathbb{I}_{b_{n}}\right)=d_{B} b_{n}, \quad q_{V_{0}} \mathbb{I}_{b_{n}}=p \mathbb{I}_{b_{n}}=\varepsilon \mathbb{I}_{b_{n}}=b_{n}, \quad q_{V_{0}} \mathbb{I}_{\beta_{n}}=\beta_{n} .
$$

We thus obtain, see Lemma 17, a morphism $q_{V_{0}} \in \operatorname{DGDM}\left(V_{0}, B\right)$ - which uniquely extends to a morphism $q_{V_{0}} \in \operatorname{DGDA}\left(\mathcal{S} V_{0}, B\right)$. Finally,

$$
q_{0}=\mu_{B} \circ\left(\phi \otimes q_{V_{0}}\right) \in \operatorname{DGDA}\left(R_{0}, B\right),
$$

where $\mu_{B}$ denotes the multiplication in $B$. Let us emphasize that $R_{U}=A \otimes \mathcal{S} U$ is a direct summand of $R_{0}=A \otimes \mathcal{S} V_{0}$, and that $\delta_{0}$ and $q_{0}$ just extend the corresponding morphisms on $R_{U}:\left.\delta_{0}\right|_{R_{U}}=d_{1}$ and $\left.q_{0}\right|_{R_{U}}=p$.

So far we ensured that $H\left(q_{0}\right): H\left(R_{0}\right) \rightarrow H(B)$ is surjective; however, it must be injective as well, i.e., for any $\sigma_{n} \in \operatorname{ker} \delta_{0}, n \geq 0$, such that $H\left(q_{0}\right)\left[\sigma_{n}\right]=0$, i.e., such that $q_{0} \sigma_{n} \in \operatorname{im} d_{B}$, there should exist $\sigma_{n+1} \in R_{0}$ such that

$$
\sigma_{n}=\delta_{0} \sigma_{n+1} .
$$

We denote by $\mathcal{B}_{0}$ the set of $\delta_{0}$-cycles that are sent to $d_{B}$-boundaries by $q_{0}$ :

$$
\mathcal{B}_{0}=\left\{\sigma_{n} \in \operatorname{ker} \delta_{0}: q_{0} \sigma_{n} \in \operatorname{im} d_{B}, n \geq 0\right\} .
$$


In principle it now suffices to add, to the generators of $V_{0}$, generators $\mathbb{I}_{\sigma_{n}}^{1}$ of degree $n+1$, $\sigma_{n} \in \mathcal{B}_{0}$, and to extend the differential $\delta_{0}$ on $R_{0}$ so that the differential of $\mathbb{I}_{\sigma_{n}}^{1}$ coincides with $\sigma_{n}\left(\triangleright_{2}\right)$. However, it turns out that to obtain a functorial 'Cof - TrivFib' decomposition, we must add a new generator $\mathbb{I}_{\sigma_{n}, \mathfrak{b}_{n+1}}^{1}$ of degree $n+1$, for each pair $\left(\sigma_{n}, \mathfrak{b}_{n+1}\right)$ such that $\sigma_{n} \in \operatorname{ker} \delta_{0}$ and $q_{0} \sigma_{n}=d_{B} \mathfrak{b}_{n+1}$ : we set

$$
\mathfrak{B}_{0}=\left\{\left(\sigma_{n}, \mathfrak{b}_{n+1}\right): \sigma_{n} \in \operatorname{ker} \delta_{0}, \mathfrak{b}_{n+1} \in d_{B}^{-1}\left\{q_{0} \sigma_{n}\right\}, n \geq 0\right\}
$$

and

$$
V_{1}:=V_{0} \oplus G_{1}:=V_{0} \oplus\left\langle\mathbb{I}_{\sigma_{n}, \mathfrak{b}_{n+1}}^{1}:\left(\sigma_{n}, \mathfrak{b}_{n+1}\right) \in \mathfrak{B}_{0}\right\rangle .
$$

To endow the graded $\mathcal{D}$-algebra

$$
R_{1}:=A \otimes \mathcal{S} V_{1} \simeq R_{0} \otimes \mathcal{S} G_{1}
$$

with a differential graded $\mathcal{D}$-algebra structure $\delta_{1}$, we apply Lemma 23, with

$$
\delta_{1}\left(\mathbb{I}_{\sigma_{n}, \mathfrak{b}_{n+1}}^{1}\right)=\sigma_{n} \in\left(R_{0}\right)_{n} \cap \operatorname{ker} \delta_{0},
$$

exactly as suggested by Equation (62). The differential $\delta_{1}$ is then given by Equation (23) and it extends the differential $\delta_{0}$ on $R_{0}$. The extension of the DGDA-morphism $q_{0}: R_{0} \rightarrow B$ by a DGDA-morphism $q_{1}: R_{1} \rightarrow B$ is built from its definition

$$
q_{1}\left(\mathbb{I}_{\sigma_{n}, \mathfrak{b}_{n+1}}^{1}\right)=\mathfrak{b}_{n+1} \in B_{n+1} \cap d_{B}^{-1}\left\{q_{0} \delta_{1}\left(\mathbb{I}_{\sigma_{n}, \mathfrak{b}_{n+1}}^{1}\right)\right\}
$$

on the generators and from Equation (34) in Lemma 23 ,

Finally, starting from $\left(R_{U}, d_{1}\right) \in \mathrm{DGDA}$ and $p \in \mathrm{DGDA}\left(R_{U}, B\right)$, we end up - when trying to make $H(p)$ bijective - with $\left(R_{1}, \delta_{1}\right) \in \mathrm{DGDA}$ and $q_{1} \in \mathrm{DGDA}\left(R_{1}, B\right)$ - so that the question is whether $H\left(q_{1}\right): H\left(R_{1}\right) \rightarrow H(B)$ is bijective or not. Since $\left(R_{1}, \delta_{1}\right)$ extends $\left(R_{0}, \delta_{0}\right)$ and $H\left(q_{0}\right): H\left(R_{0}\right) \rightarrow H(B)$ is surjective, it is easily checked that this property holds a fortiori for $H\left(q_{1}\right)$. However, when working with $R_{1} \supset R_{0}$, the 'critical set' $\mathcal{B}_{1} \supset \mathcal{B}_{0}$ increases, so that we must add new generators $\mathbb{I}_{\sigma_{n}}^{2}, \sigma_{n} \in \mathcal{B}_{1} \backslash \mathcal{B}_{0}$, where

$$
\mathcal{B}_{1}=\left\{\sigma_{n} \in \operatorname{ker} \delta_{1}: q_{1} \sigma_{n} \in \operatorname{im} d_{B}, n \geq 0\right\} . \quad\left(\triangleright_{3}\right)
$$

To build a functorial factorization, we consider not only the 'critical set'

$$
\mathfrak{B}_{1}=\left\{\left(\sigma_{n}, \mathfrak{b}_{n+1}\right): \sigma_{n} \in \operatorname{ker} \delta_{1}, \mathfrak{b}_{n+1} \in d_{B}^{-1}\left\{q_{1} \sigma_{n}\right\}, n \geq 0\right\},
$$

but also the module of new generators

$$
G_{2}=\left\langle\mathbb{I}_{\sigma_{n}, \mathfrak{b}_{n+1}}^{2}:\left(\sigma_{n}, \mathfrak{b}_{n+1}\right) \in \mathfrak{B}_{1}\right\rangle,
$$

indexed, not by $\mathfrak{B}_{1} \backslash \mathfrak{B}_{0}$, but by $\mathfrak{B}_{1}$. Hence an iteration of the procedure (63) - (67) and the definition of a sequence

$$
\left(R_{0}, \delta_{0}\right) \rightarrow\left(R_{1}, \delta_{1}\right) \rightarrow\left(R_{2}, \delta_{2}\right) \rightarrow \ldots \rightarrow\left(R_{k-1}, \delta_{k-1}\right) \rightarrow\left(R_{k}, \delta_{k}\right) \rightarrow \ldots
$$


of canonical inclusions of differential graded $\mathcal{D}$-algebras $\left(R_{k}, \delta_{k}\right), R_{k}=A \otimes \mathcal{S} V_{k},\left.\delta_{k}\right|_{R_{k-1}}=\delta_{k-1}$, together with a sequence of DGDA-morphisms $q_{k}: R_{k} \rightarrow B$, such that $\left.q_{k}\right|_{R_{k-1}}=q_{k-1}$. The definitions of the differentials $\delta_{k}$ and the morphisms $q_{k}$ are obtained inductively, and are based on Lemma 23, as well as on equations of the same type as (66) and (67).

The direct limit of this sequence is a differential graded $\mathcal{D}$-algebra $\left(R_{V}, d_{2}\right)=\left(A \otimes \mathcal{S} V, d_{2}\right)$, together with a morphism $q: A \otimes \mathcal{S} V \rightarrow B$.

As a set, the colimit of the considered system of canonically included algebras $\left(R_{k}, \delta_{k}\right)$, is just the union of the sets $R_{k}$, see Equation (50). We proved above that this set-theoretical inductive limit can be endowed in the standard manner with a differential graded $\mathcal{D}$-algebra structure and that the resulting algebra is the direct limit in DGDA. One thus obtains in particular that $\left.d_{2}\right|_{R_{k}}=\delta_{k}$.

Finally, the morphism $q: R_{V} \rightarrow B$ comes from the universality property of the colimit and it allows us to factor the morphisms $q_{k}: R_{k} \rightarrow B$ through $R_{V}$. We have: $\left.q\right|_{R_{k}}=q_{k}$.

We will show that this morphism $A \otimes \mathcal{S} V \stackrel{q}{\longrightarrow} B$ really leads to a 'Cof - TrivFib' decomposition $A \stackrel{j}{\longrightarrow} A \otimes \mathcal{S} V \stackrel{q}{\longrightarrow} B$ of $A \stackrel{\phi}{\longrightarrow} B$.

Theorem 29. In DGDA, a functorial 'TrivCof - Fib' factorization $(i, p)$ and a functorial 'Cof - TrivFib' factorization $(j, q)$ of an arbitrary morphism

$$
\phi:\left(A, d_{A}\right) \rightarrow\left(B, d_{B}\right)
$$

see Figure 4, can be constructed as follows:

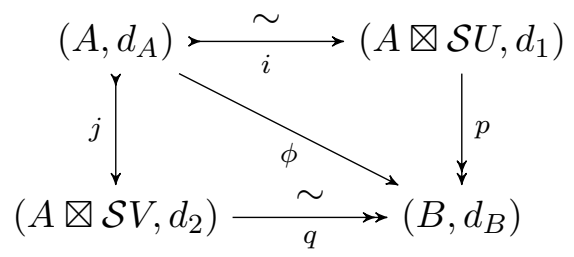

Figure 4: Functorial factorizations

(1) The module $U$ is the free non-negatively graded $\mathcal{D}$-module with homogeneous basis

$$
\bigcup\left\{s^{-1} \mathbb{I}_{b_{n}}, \mathbb{I}_{b_{n}}\right\}
$$

where the union is over all $b_{n} \in B_{n}$ and all $n>0$, and where $\operatorname{deg}\left(s^{-1} \mathbb{I}_{b_{n}}\right)=n-1$ and $\operatorname{deg}\left(\mathbb{I}_{b_{n}}\right)=n$. In other words, the module $U$ is a direct sum of copies of the discs

$$
D^{n}=\mathcal{D} \cdot \mathbb{I}_{b_{n}} \oplus \mathcal{D} \cdot s^{-1} \mathbb{I}_{b_{n}},
$$

$n>0$. The differentials

$$
s^{-1}: D^{n} \ni \mathbb{I}_{b_{n}} \rightarrow s^{-1} \mathbb{I}_{b_{n}} \in D^{n}
$$


induce a differential $d_{U}$ in $U$, which in turn implements a differential $d_{S}$ in $\mathcal{S} U$. The differential $d_{1}$ is then given by $d_{1}=d_{A} \otimes \mathrm{id}+\mathrm{id} \otimes d_{S}$. The trivial cofibration $i: A \rightarrow A \otimes \mathcal{S} U$ is a split $R S D A$ defined by $i: \mathfrak{a} \mapsto \mathfrak{a} \otimes 1_{\mathcal{O}}$, and the fibration $p: A \otimes \mathcal{S} U \rightarrow B$ is defined by $p=\mu_{B} \circ(\phi \otimes \varepsilon)$, where $\mu_{B}$ is the multiplication of $B$ and where $\varepsilon\left(\mathbb{I}_{b_{n}}\right)=b_{n}$ and $\varepsilon\left(s^{-1} \mathbb{I}_{b_{n}}\right)=d_{B} b_{n}$.

(2) The module $V$ is the free non-negatively graded $\mathcal{D}$-module with homogeneous basis

$$
\bigcup\left\{s^{-1} \mathbb{I}_{b_{n}}, \mathbb{I}_{b_{n}}, \mathbb{I}_{\beta_{n}}, \mathbb{I}_{\sigma_{n}, \mathfrak{b}_{n+1}}^{1}, \mathbb{I}_{\sigma_{n}, \mathfrak{b}_{n+1}}^{2}, \ldots, \mathbb{I}_{\sigma_{n}, \mathfrak{b}_{n+1}}^{k}, \ldots\right\},
$$

where the union is over all $b_{n} \in B_{n}, n>0$, all $\beta_{n} \in \operatorname{ker}_{n} d_{B}, n \geq 0$, and all pairs

$$
\left(\sigma_{n}, \mathfrak{b}_{n+1}\right), n \geq 0, \text { in } \mathfrak{B}_{0}, \mathfrak{B}_{1}, \ldots, \mathfrak{B}_{k}, \ldots
$$

respectively. The sequence of sets

$$
\mathfrak{B}_{k-1}=\left\{\left(\sigma_{n}, \mathfrak{b}_{n+1}\right): \sigma_{n} \in \operatorname{ker} \delta_{k-1}, \mathfrak{b}_{n+1} \in d_{B}^{-1}\left\{q_{k-1} \sigma_{n}\right\}, n \geq 0\right\}
$$

is defined inductively, together with an increasing sequence of differential graded $\mathcal{D}$-algebras $\left(A \otimes \mathcal{S} V_{k}, \delta_{k}\right)$ and a sequence of morphisms $q_{k}: A \otimes \mathcal{S} V_{k} \rightarrow B$, by means of formulas of the type (63) - (67) (see also (57) - (61)). The degrees of the generators of $V$ are

$$
n-1, n, n, n+1, n+1, \ldots, n+1, \ldots
$$

The differential graded $\mathcal{D}$-algebra $\left(A \otimes \mathcal{S} V, d_{2}\right)$ is the colimit of the preceding increasing sequence of algebras:

$$
\left.d_{2}\right|_{A \otimes \mathcal{S} V_{k}}=\delta_{k} .
$$

The trivial fibration $q: A \otimes \mathcal{S} V \rightarrow B$ is induced by the $q_{k}-s$ via universality of the colimit:

$$
\left.q\right|_{A \otimes \mathcal{S} V_{k}}=q_{k} .
$$

Finally, the cofibration $j: A \rightarrow A \otimes \mathcal{S} V$ is a (non-split) RSDA, which is defined as in (1) as the canonical inclusion; the canonical inclusion $j_{k}: A \rightarrow A \otimes \mathcal{S} V_{k}, k>0$, is also a (non-split) $R S D A$, whereas $j_{0}: A \rightarrow A \otimes \mathcal{S} V_{0}$ is a split RSDA.

Proof. See Appendix 11.6.

Remark 30. - If we are content with a non-functorial 'Cof - TrivFib' factorization, we may consider the colimit $A \otimes \mathcal{S} \mathcal{V}$ of the sequence $A \otimes \mathcal{S} \mathcal{V}_{k}$ that is obtained by adding only generators (see $\left.\left(\triangleright_{1}\right)\right)$

$$
\mathbb{I}_{\dot{\beta}_{n}}, n \geq 0, \dot{\beta}_{n} \simeq\left[\beta_{n}\right] \in H_{n}(B),
$$

and by adding only generators (see $\left(\triangleright_{2}\right)$ and $\left.\left(\triangleright_{3}\right)\right)$

$$
\mathbb{I}_{\sigma_{n}}^{1}, \mathbb{I}_{\sigma_{n}}^{2}, \ldots, n \geq 0, \sigma_{n} \in \mathcal{B}_{0}, \mathcal{B}_{1} \backslash \mathcal{B}_{0}, \ldots
$$

- An explicit description of the functorial fibrant and cofibrant replacement functors, induced by the 'TrivCof - Fib' and 'Cof - TrivFib' decompositions of Theorem 29, can be found in Appendix 11.7 


\section{First remarks on Koszul-Tate resolutions}

In this last section, we provide a first insight into Koszul-Tate resolutions. The Koszul-Tate resolution (KTR), which is used in Mathematical Physics and more precisely in [Bar10], relies on horizontal differential operators, whose coordinate expression contains total derivatives. For instance, in the case of a unique base coordinate $t$, the total derivative with respect to $t$ is

$$
D_{t}=\partial_{t}+\dot{q} \partial_{q}+\ddot{q} \partial_{\dot{q}}+\ldots,
$$

where $q, \dot{q}, \ddot{q}, \ldots$ are infinite jet bundle fiber coordinates. The main concept of the jet bundle formalism is the Cartan connection $\mathcal{C}$, which allows to lift base differential operators $\partial_{t}$ acting on base functions $\mathcal{O}=\mathcal{O}(X)$ to horizontal differential operators $D_{t}$ acting on the functions $\mathcal{O}\left(J^{\infty} E\right)$ of the infinite jet bundle $J^{\infty} E \rightarrow X$ of a vector bundle $E \rightarrow X$. Hence, the total derivative $D_{t}^{k} F$ of a jet bundle function $F$ can be viewed as the action $\partial_{t}^{k} \cdot F$ on $F$ of the corresponding base-derivative. In other words, one defines this action as the natural action by the corresponding lifted operator. Jet bundle functions $\mathcal{O}\left(J^{\infty} E\right)$ thus become an algebra

$$
\mathcal{O}\left(J^{\infty} E\right) \in \mathcal{D A}
$$

over $\mathcal{D}=\mathcal{D}(X)$. In our algebraic geometric setting, there exists an infinite jet bundle functor $\mathcal{J}^{\infty}: \mathcal{O A} \rightarrow \mathcal{D A}$, which transforms the algebra $\mathcal{O}(E) \in \mathcal{O}$ A of vector bundle functions into an algebra

$$
\mathcal{J}^{\infty}(\mathcal{O}(E)) \in \mathcal{D A} .
$$

The latter is the algebraic geometric counterpart of the $\mathcal{D}$-algebra $\mathcal{O}\left(J^{\infty} E\right)$ used in smooth geometry. Recall now that (the prolongation of) a partial differential equation on the sections of $E$ can be viewed as an algebraic equation on the points of $J^{\infty} E$. The solutions of the latter form the critical surface $\Sigma \subset J^{\infty} E$. The function algebra

$$
\mathcal{O}\left(J^{\infty} E\right) / I(\Sigma) \in \mathcal{D A}
$$

of this stationary surface $\Sigma$ is the quotient of the $\mathcal{D}$-algebra $\mathcal{O}\left(J^{\infty} E\right)$ by the $\mathcal{D}$-ideal $I(\Sigma)$ of those jet bundle functions that vanish on $\Sigma$. The Koszul-Tate resolution resolves this quotient. Now, for any $\mathcal{D}$-ideal $\mathcal{I}$, we think about

$$
\mathcal{J}^{\infty}(\mathcal{O}(E)) / \mathcal{I} \in \mathcal{D A}
$$

as the function algebra of some critical locus $\Sigma$. In our model categorical context, its (KoszulTate) resolution should be related to a cofibrant replacement of $\mathcal{J}^{\infty}(\mathcal{O}(E)) / \mathcal{I} \in \mathcal{D}$ A in the model category DGDA. This will be explained in detail below. Let us stress again, before proceeding, that in the present model categorical setting, the algebra $\mathcal{D}$ is the algebra $\mathcal{D}_{X}(X)$ of global sections of the sheaf $\mathcal{D}_{X}$, where $X$ is a smooth affine algebraic variety.

In a separate paper [PP17a], we will give a new, general, and precise definition of KoszulTate resolutions. Instead of defining, as in homological algebra, a KTR for the quotient of some type of ring by an ideal, we will consider a (sheaf of) $\mathcal{D}_{X}$-algebra(s) $\mathcal{A}$ over an arbitrary smooth 
scheme $X$, as well as a differential graded $\mathcal{D}_{X}$-algebra (sheaf) morphism $\phi: \mathcal{A} \rightarrow \mathcal{B}$. We will denote by $\mathcal{A}\left[\mathcal{D}_{X}\right]$ the ring of differential operators on $X$ with coefficients in $\mathcal{A}$ and will define a $\mathcal{D}$-geometric KTR of $\phi$ as a differential graded $\mathcal{A}\left[\mathcal{D}_{X}\right]$-algebra morphism $\psi: \mathcal{C} \rightarrow \mathcal{B}$, whose definition mimics the essential characteristics of our model categorical or cofibrant replacement KTR here above. It will turn out that such a KTR does always exist. We will further show that the KTR of a quotient ring [Tat57], the KTR used in Mathematical Physics [HT92], the KTR implemented by a compatibility complex [Ver02], as well as our model categorical KTR, are all $\mathcal{D}$-geometric Koszul-Tate resolutions. We will actually give precise comparison results for these Koszul-Tate resolutions, thus providing a kind of dictionary between different fields of science and their specific languages.

Hence, the present section should be viewed as an introduction to topics on which we will elaborate in PP17a.

\subsection{Undercategories of model categories}

When recalling that the coproduct in DGDA is the tensor product, we get from Hir05] that:

Proposition 31. For any differential graded $\mathcal{D}$-algebra $A$, the coslice category $A \downarrow$ DGDA carries a cofibrantly generated model structure given by the adjoint pair $L_{\otimes}:$ DGDA $\rightleftarrows A \downarrow$ DGDA : For, in the sense that its distinguished morphism classes are defined by For and its generating cofibrations and generating trivial cofibrations are given by $L_{\otimes}$.

\subsection{Basics of jet bundle formalism}

The jet bundle formalism allows for a coordinate-free approach to partial differential equations (PDE-s), i.e., to (not necessarily linear) differential operators (DO-s) acting between sections of smooth vector bundles (the confinement to vector bundles does not appear in more advanced approaches). To uncover the main ideas, we implicitly consider in this subsection trivialized line bundles $E$ over a 1-dimensional manifold $X$, i.e., we assume that $E \simeq \mathbb{R} \times \mathbb{R}$.

The key-aspect of the jet bundle approach to PDE-s is the passage to purely algebraic equations. Consider the order $k$ differential equation (DE)

$$
F\left(t, \phi(t), d_{t} \phi, \ldots, d_{t}^{k} \phi\right)=\left.F\left(t, \phi, \phi^{\prime}, \ldots, \phi^{(k)}\right)\right|_{j^{k} \phi}=0,
$$

where $\left(t, \phi, \phi^{\prime}, \ldots, \phi^{(k)}\right)$ are coordinates of the $k$-th jet space $J^{k} E$ and where $j^{k} \phi$ is the $k$-jet of the section $\phi(t)$. Note that the algebraic equation

$$
F\left(t, \phi, \phi^{\prime}, \ldots, \phi^{(k)}\right)=0
$$

defines a 'surface' $\mathcal{E}^{k} \subset J^{k} E$, and that a solution of the considered DE is nothing but a section $\phi(t)$ whose $k$-jet is located on $\mathcal{E}^{k}$.

A second fundamental feature is that one prefers replacing the original system of PDE-s by an enlarged system, its infinite prolongation, which also takes into account the consequences 
of the original one. More precisely, if $\phi(t)$ satisfies the original PDE, we have also

$$
\begin{gathered}
d_{t}^{\ell}\left(F\left(t, \phi(t), d_{t} \phi, \ldots, d_{t}^{k} \phi\right)\right)=\left.\left(\partial_{t}+\phi^{\prime} \partial_{\phi}+\phi^{\prime \prime} \partial_{\phi^{\prime}}+\ldots\right)^{\ell} F\left(t, \phi, \phi^{\prime}, \ldots, \phi^{(k)}\right)\right|_{j^{\infty} \phi}=: \\
\left.D_{t}^{\ell} F\left(t, \phi, \phi^{\prime}, \ldots, \phi^{(k)}\right)\right|_{j^{\infty} \phi}=0, \quad \forall \ell \in \mathbb{N} .
\end{gathered}
$$

Let us stress that the 'total derivative' $D_{t}$ or horizontal lift $D_{t}$ of $d_{t}$ is actually an infinite sum. The two systems of PDE-s, (73) and (75), have clearly the same solutions, so we may focus just as well on (75). The corresponding algebraic system

$$
D_{t}^{\ell} F\left(t, \phi, \phi^{\prime}, \ldots, \phi^{(k)}\right)=0, \forall \ell \in \mathbb{N}
$$

defines a 'surface' $\mathcal{E}^{\infty}$ in the infinite jet bundle $\pi_{\infty}: J^{\infty} E \rightarrow X$. A solution of the original system (73) is now a section $\phi \in \Gamma(X, E)$ such that $\left(j^{\infty} \phi\right)(X) \subset \mathcal{E}^{\infty}$. The 'surface' $\mathcal{E}^{\infty}$ is often referred to as the 'stationary surface' or the 'shell'.

The just described passage from prolonged PDE-s to prolonged algebraic equations involves the lift of differential operators $d_{t}^{\ell}$ acting on $\mathcal{O}(X)=\Gamma(X, X \times \mathbb{R})$ (resp., sending - more generally - sections $\Gamma(X, G)$ of some vector bundle to sections $\Gamma(X, K))$, to horizontal differential operators $D_{t}^{\ell}$ acting on $\mathcal{O}\left(J^{\infty} E\right)$ (resp., acting from $\Gamma\left(J^{\infty} E, \pi_{\infty}^{*} G\right)$ to $\left.\Gamma\left(J^{\infty} E, \pi_{\infty}^{*} K\right)\right)$. As seen from Equation (75), this lift is defined by

$$
\left(D_{t}^{\ell} F\right) \circ j^{\infty} \phi=d_{t}^{\ell}\left(F \circ j^{\infty} \phi\right)
$$

(note that composites of the type $F \circ j^{\infty} \phi$, where $F$ is a section of the pullback bundle $\pi_{\infty}^{*} G$, are sections of $G$ ). The interesting observation is that the jet bundle formalism naturally leads to a systematic base change $X \rightsquigarrow J^{\infty} E$. The remark is fundamental in the sense that both the classical Koszul-Tate resolution (i.e., the Tate extension of the Koszul resolution of a regular surface) and Verbovetsky's Koszul-Tate resolution (i.e., the resolution induced by the compatibility complex of the linearization of the equation), use the jet formalism to resolve on-shell functions $\mathcal{O}\left(\mathcal{E}^{\infty}\right)$, and thus contain the base change $\bullet \rightarrow X \rightsquigarrow \bullet \rightarrow J^{\infty} E$. This means, dually, that we pass from DGDA, i.e., from the coslice category $\mathcal{O}(X) \downarrow$ DGDA to the coslice category $\mathcal{O}\left(J^{\infty} E\right) \downarrow D G D A$.

\subsection{Revisiting classical Koszul-Tate resolution}

We first recall the local construction of the Koszul resolution of the function algebra $\mathcal{O}(\Sigma)$ of a regular surface $\Sigma \subset \mathbb{R}^{n}$. Such a surface $\Sigma$, say of codimension $r$, can locally always be described - in appropriate coordinates - by the equations

$$
\Sigma: x^{a}=0, \forall a \in\{1, \ldots, r\}
$$

The Koszul resolution of $\mathcal{O}(\Sigma)$ is then the chain complex made of the free Grassmann algebra, i.e., the free graded commutative algebra

$$
\mathrm{K}=\mathcal{O}\left(\mathbb{R}^{n}\right) \otimes \mathcal{S}\left[\phi^{a *}\right]
$$


on $r$ odd generators $\phi^{a *}$ - associated to the equations (77) - and of the Koszul differential

$$
\delta_{\mathrm{K}}=x^{a} \partial_{\phi^{a *}} .
$$

Of course, the claim that this complex is a resolution of $\mathcal{O}(\Sigma)$ means that the homology of $\left(\mathrm{K}, \delta_{\mathrm{K}}\right)$ is given by

$$
H_{0}(\mathrm{~K})=\mathcal{O}(\Sigma) \quad \text { and } \quad H_{k}(\mathrm{~K})=0, \forall k>0
$$

The Koszul-Tate resolution of the algebra $\mathcal{O}\left(\mathcal{E}^{\infty}\right)$ of on-shell functions is a generalization of the preceding Koszul resolution. In gauge field theory (our main target), $\mathcal{E}^{\infty}$ is the stationary surface given by a system

$$
\mathcal{E}^{\infty}: D_{x}^{\alpha} F_{i}=0, \forall \alpha, i
$$

of prolonged algebraized (see (76)) Euler-Lagrange equations that correspond to some action functional (here $x \in \mathbb{R}^{p}$ and $\alpha \in \mathbb{N}^{p}$ ). However, there is a difference between the situations (77) and (80): in the latter, there exist gauge symmetries that implement Noether identities and their extensions - i.e., extensions

$$
D_{x}^{\beta} G_{j \alpha}^{i} D_{x}^{\alpha} F_{i}=0, \forall \beta, j
$$

of $\mathcal{O}\left(J^{\infty} E\right)$-linear relations $G_{j \alpha}^{i} D_{x}^{\alpha} F_{i}=0$ between the equations $D_{x}^{\alpha} F_{i}=0$ of $\mathcal{E}^{\infty}$, which do not have any counterpart in the former. It turns out that, to kill the homology (see (79) ), we must introduce additional generators that take into account these relations. More precisely, we do not only associate degree 1 generators $\phi_{i}^{\alpha *}$ to the equations (80), but assign further degree 2 generators $C_{j}^{\beta *}$ to the relations (81). The Koszul-Tate resolution of $\mathcal{O}\left(\mathcal{E}^{\infty}\right)$ is then (under appropriate irreducibility and regularity conditions) the chain complex, whose chains are the elements of the free Grassmann algebra

$$
\mathrm{KT}=\mathcal{O}\left(J^{\infty} E\right) \otimes \mathcal{S}\left[\phi_{i}^{\alpha *}, C_{j}^{\beta *}\right],
$$

and whose differential is defined in analogy with (78) by

$$
\delta_{\mathrm{KT}}=D_{x}^{\alpha} F_{i} \partial_{\phi_{i}^{\alpha *}}+D_{x}^{\beta} G_{j \alpha}^{i} D_{x}^{\alpha} \phi_{i}^{*} \partial_{C_{j}^{\beta *}},
$$

where we substituted $\phi_{i}^{*}$ to $F_{i}$ (and where total derivatives have to be interpreted in the extended sense that puts the 'antifields' $\phi_{i}^{*}$ and $C_{j}^{*}$ on an equal footing with the 'fields' $\phi^{i}$ (fiber coordinates of $E$ ), i.e., where we set

$$
\left.D_{x^{k}}=\partial_{x^{k}}+\phi_{k \alpha}^{i} \partial_{\phi_{\alpha}^{i}}+\phi_{i}^{k \alpha *} \partial_{\phi_{i}^{\alpha *}}+C_{j}^{k \beta *} \partial_{C_{j}^{\beta *}}\right) .
$$

The homology of this Koszul-Tate chain complex is actually concentrated in degree 0, where it coincides with $\mathcal{O}\left(\mathcal{E}^{\infty}\right)$ (compare with (79) ) [HT92]. 


\section{$10.4 \mathcal{D}$-algebraic version of the Koszul-Tate resolution}

In this subsection, we briefly report on the $\mathcal{D}$-algebraic approach to 'Koszul-Tate' (see PP17a for additional details).

Proposition 32. The functor

$$
\text { For }: \mathcal{D A} \rightarrow \mathcal{O A}
$$

has a left adjoint

$$
\mathcal{J}^{\infty}: \mathcal{O A} \rightarrow \mathcal{D A}
$$

i.e., for $B \in \mathcal{O A}$ and $A \in \mathcal{D A}$, we have

$$
\operatorname{Hom}_{\mathcal{D A}}\left(\mathcal{J}^{\infty}(B), A\right) \simeq \operatorname{Hom}_{\mathcal{O A}}(B, \operatorname{For}(A)),
$$

functorially in $A, B$.

Let now $\pi: E \rightarrow X$ be a smooth map of smooth affine algebraic varieties (or a smooth vector bundle). The function algebra $B=\mathcal{O}(E)$ (in the vector bundle case, we only consider those smooth functions on $E$ that are polynomial along the fibers, i.e., $\mathcal{O}(E):=\Gamma\left(\mathcal{S} E^{*}\right)$ ) is canonically an $\mathcal{O}$-algebra, so that the jet algebra $\mathcal{J}^{\infty}(\mathcal{O}(E))$ is a $\mathcal{D}$-algebra. The latter can be thought of as the $\mathcal{D}$-algebraic counterpart of $\mathcal{O}\left(J^{\infty} E\right)$. Just as we considered above a scalar PDE with unknown in $\Gamma(E)$ as a function $F \in \mathcal{O}\left(J^{\infty} E\right)$ (see (74)), an element $P \in \mathcal{J}^{\infty}(\mathcal{O}(E))$ can be viewed as a polynomial PDE acting on sections of $\pi: E \rightarrow X$. Finally, the $\mathcal{D}$-algebraic version of on-shell functions $\mathcal{O}\left(\mathcal{E}^{\infty}\right)=\mathcal{O}\left(J^{\infty} E\right) /(F)$ is the quotient $\mathcal{R}(E, P):=\mathcal{J}^{\infty}(\mathcal{O}(E)) /(P)$ of the jet $\mathcal{D}$-algebra by the $\mathcal{D}$-ideal $(P)$.

A first candidate for a Koszul-Tate resolution of $\mathcal{R}:=\mathcal{R}(E, P) \in \mathcal{D}$ A is of course the cofibrant replacement of $\mathcal{R}$ in DGDA given by the functorial 'Cof - TrivFib' factorization of Theorem 29, when applied to the canonical DGDA-morphism $\mathcal{O} \rightarrow \mathcal{R}$. Indeed, this decomposition implements a functorial cofibrant replacement functor $Q$ (see Theorem 35 below) with value $Q(\mathcal{R})=\mathcal{S} V$ described in Theorem 29.

$$
\mathcal{O} \longmapsto \mathcal{S} V \stackrel{\sim}{\rightarrow} \mathcal{R} .
$$

Since $\mathcal{R}$ is concentrated in degree 0 and has 0 differential, it is clear that $H_{k}(\mathcal{S} V)$ vanishes, except in degree 0 where it coincides with $\mathcal{R}$.

As already mentioned, we propose a general and precise definition of a Koszul-Tate resolution in PP17a]. Although such a definition does not seem to exist in the literature, the classical Koszul-Tate resolution of the quotient of a commutative ring $k$ by an ideal $I$ is a $k$-algebra that resolves $k / I$.

The natural idea - to get a $\mathcal{J}^{\infty}(\mathcal{O}(E))$-algebra - is to replace $\mathcal{S} V$ by $\mathcal{J}^{\infty}(\mathcal{O}(E)) \otimes \mathcal{S} V$, and, more precisely, to consider the 'Cof - TrivFib' decomposition

$$
\mathcal{J}^{\infty}(\mathcal{O}(E)) \longmapsto \mathcal{J}^{\infty}(\mathcal{O}(E)) \otimes \mathcal{S} V \stackrel{\sim}{\rightarrow} \mathcal{J}^{\infty}(\mathcal{O}(E)) /(P) .
$$


The DGDA

$$
\mathcal{J}^{\infty}(\mathcal{O}(E)) \otimes \mathcal{S} V
$$

is a $\mathcal{J}^{\infty}(\mathcal{O}(E))$-algebra that resolves $\mathcal{R}=\mathcal{J}^{\infty}(\mathcal{O}(E)) /(P)$, but it is of course not a cofibrant replacement, since the left algebra is not the initial object $\mathcal{O}$ in DGDA (further, the considered factorization does not canonically induce a cofibrant replacement in DGDA, since it can be shown that the morphism $\mathcal{O} \rightarrow \mathcal{J}^{\infty}(\mathcal{O}(E))$ is not a cofibration). However, as emphasized above, the Koszul-Tate problem requires a passage from DGDA to $\mathcal{J}^{\infty}(\mathcal{O}(E)) \downarrow$ DGDA. It is easily checked that, in the latter undercategory, $\mathcal{J}^{\infty}(\mathcal{O}(E)) \otimes \mathcal{S} V$ is a cofibrant replacement of $\mathcal{J}^{\infty}(\mathcal{O}(E)) /(P)$. To further illuminate the $\mathcal{D}$-algebraic approach to Koszul-Tate, let us mention why the complex (82) is of the same type as (85). Just as the variables $\phi^{(k)}$ (see (73)) are algebraizations of the derivatives $d_{t}^{k} \phi$ of a section $\phi$ of a vector bundle $E \rightarrow X$ (fields), the generators $\phi_{i}^{\alpha *}$ and $C_{j}^{\beta *}$ (see (80) and (810) symbolize the total derivatives $D_{x}^{\alpha} \phi_{i}^{*}$ and $D_{x}^{\beta} C_{j}^{*}$ of sections $\phi^{*}$ and $C^{*}$ of some vector bundles $\pi_{\infty}^{*} F_{1} \rightarrow J^{\infty} E$ and $\pi_{\infty}^{*} F_{2} \rightarrow J^{\infty} E$ (antifields). Hence, the $\phi_{i}^{\alpha *}$ and $C_{j}^{\beta *}$ can be thought of as the horizontal jet bundle coordinates of $\pi_{\infty}^{*} F_{1}$ and $\pi_{\infty}^{*} F_{2}$. These coordinates may of course be denoted by other symbols, e.g., by $\partial_{x}^{\alpha} \cdot \phi_{i}^{*}$ and $\partial_{x}^{\beta} \cdot C_{j}^{*}$, provided we define the $\mathcal{D}$-action $\cdot$ as the action $D_{x}^{\alpha} \phi_{i}^{*}$ and $D_{x}^{\beta} C_{j}^{*}$ by the corresponding horizontal lift, so that we get appropriate interpretations when the $\phi_{i}^{*}$-s and the $C_{j}^{*}$-s are the components of true sections. This convention allows us to write

$$
\mathrm{KT}=J \otimes \mathcal{S}\left[\partial_{x}^{\alpha} \cdot \phi_{i}^{*}, \partial_{x}^{\beta} \cdot C_{j}^{*}\right]=J \otimes_{\mathcal{O}} \mathcal{S}_{\mathcal{O}}\left(\oplus_{i} \mathcal{D} \cdot \phi_{i}^{*} \bigoplus \oplus_{j} \mathcal{D} \cdot C_{j}^{*}\right),
$$

where $J=\mathcal{J}^{\infty}(\mathcal{O}(E))$, so that the space (82) is really of the type (85). Let us emphasize that (82) and (85), although of the same type, are of course not equal (for instance, the classical Koszul-Tate resolution is far from being functorial). For further details, see [PP17a].

\section{Appendices}

The following appendices do not contain new results but might have a pedagogical value. Various (also online) sources were used. Notation is the same as in the main part of the text.

\subsection{Appendix 1 - Quasi-coherent sheaves of modules}

A quasi-coherent $\mathcal{R}$-module is an object $\mathcal{P} \in \operatorname{Mod}(\mathcal{R})$ that is locally presented, i.e., for any $x \in X$, there is a neighborhood $U \ni x$, such that there is an exact sequence of sheaves

$$
\left.\left.\left.\mathcal{R}^{K_{U}}\right|_{U} \rightarrow \mathcal{R}^{J_{U}}\right|_{U} \rightarrow \mathcal{P}\right|_{U} \rightarrow 0
$$

where $\mathcal{R}^{K_{U}}$ and $\mathcal{R}^{J_{U}}$ are (not necessarily finite) direct sums. Let us recall that an infinite direct sum of sheaves need not be a sheaf, so that a sheafification is required. The category $\operatorname{qc} \operatorname{Mod}(\mathcal{R})$ of quasi-coherent $\mathcal{R}$-modules is not abelian in general, but is abelian in the context of Algebraic Geometry, i.e., if $\mathcal{R}$ is the function sheaf of a scheme. 


\subsection{Appendix $2-\mathcal{D}$-modules}

We already indicated that $\mathcal{D}$-modules are fundamental in algebraic analysis: they allow us to apply methods of homological algebra and sheaf theory to the study of systems of PDE-s [KS90].

We first explain the key idea of Proposition 1 considering - to simplify - global sections instead of sheaves.

We denote by $\mathcal{D}$ the ring of differential operators acting on functions of a suitable base space $X$, e.g., a finite-dimensional smooth manifold [Cos11]. A $\mathcal{D}$-module $M \in \operatorname{Mod}(\mathcal{D})$ (resp., $\left.M \in \operatorname{Mod}\left(\mathcal{D}^{\text {op }}\right)\right)$ is a left (resp., right) module over the noncommutative ring $\mathcal{D}$. Since $\mathcal{D}$ is generated by smooth functions $f \in \mathcal{O}$ and smooth vector fields $\theta \in \Theta$, modulo the obvious commutation relations between these types of generators, a $\mathcal{D}$-action on an $\mathcal{O}$-module $M \in$ $\operatorname{Mod}(\mathcal{O})$ is completely defined if it is given for vector fields and satisfies the natural compatibility conditions. More precisely, let

$$
\cdot: \mathcal{O} \times M \ni(f, m) \mapsto f \cdot m \in M
$$

be the $\mathcal{O}$-action, and let

$$
\nabla: \Theta \times M \ni(\theta, m) \mapsto \nabla_{\theta} m \in M
$$

be an $\mathbb{R}$-bilinear ' $\Theta$-action'. For $f \in \mathcal{O}$ and $\theta, \theta^{\prime} \in \Theta$, we then necessarily extend $\nabla$ by defining the action $\nabla_{\theta \theta^{\prime}}$ (resp., $\nabla_{\theta f}$ ) of the differential operator $\theta \theta^{\prime}=\theta \circ \theta^{\prime}$ (resp., $\theta f=\theta \circ f$ ) by

$$
\nabla_{\theta \theta^{\prime}}:=\nabla_{\theta} \nabla_{\theta^{\prime}}
$$

(resp.,

$$
\left.\nabla_{\theta f}:=\nabla_{\theta}(f \cdot-)\right)
$$

Since $f \theta=f \circ \theta$, we get the compatibility condition

$$
\nabla_{f \theta}=f \cdot \nabla_{\theta},
$$

and, as $\theta f=f \theta+\theta(f)$ (resp., $\theta \theta^{\prime}=\theta^{\prime} \theta+\left[\theta, \theta^{\prime}\right]$ ) - where $\theta(f)$ (resp., $\left[\theta, \theta^{\prime}\right]$ ) denotes the Lie derivative $L_{\theta} f$ of $f$ with respect to $\theta$ (resp., the Lie bracket of the vector fields $\theta, \theta^{\prime}$ ), we also find the compatibility relations

$$
\nabla_{\theta}(f \cdot-)=f \cdot \nabla_{\theta}+\theta(f) \cdot-
$$

(resp.,

$$
\left.\nabla_{\theta} \nabla_{\theta^{\prime}}=\nabla_{\theta^{\prime}} \nabla_{\theta}+\nabla_{\left[\theta, \theta^{\prime}\right]}\right) .
$$

Hence, if the compatibility conditions (88) - (90) hold, we defined the unique structure of left $\mathcal{D}$-module on $M$ that extends the 'action of $\Theta$ '. In view of Equations (87) - (90), a D-module structure on $M \in \operatorname{Mod}(\mathcal{O})$ is the same as a flat connection on $M$.

When resuming now our explanations given in Subsection 4.1, we understand that a morphism $\nabla$ of sheaves of $\mathbb{K}$-vector spaces satisfying the conditions (1) - (3) is exactly a family 
of $\mathcal{D}_{X}(U)$-modules $\mathcal{M}_{X}(U), U \in$ Open $_{X}$, such that the $\mathcal{D}_{X}(U)$-actions are compatible with restrictions, i.e., is exactly a $\mathcal{D}_{X}$-module structure on the considered sheaf $\mathcal{M}_{X}$ of $\mathcal{O}_{X}$-modules.

Typical examples of $\mathcal{D}$-modules are:

- $\mathcal{O} \in \operatorname{Mod}(\mathcal{D})$ with action $\nabla_{\theta}=L_{\theta}$,

- the top differential forms $\Omega^{\text {top }} \in \operatorname{Mod}\left(\mathcal{D}^{\text {op }}\right)$ with action $\nabla_{\theta}=-L_{\theta}$, and

- $\mathcal{D} \in \operatorname{Mod}(\mathcal{D}) \cap \operatorname{Mod}\left(\mathcal{D}^{\text {op }}\right)$ with action given by left and right compositions.

\subsection{Appendix 3 - Sheaves versus global sections}

In Classical Differential Geometry, the fundamental spaces (resp., operators), e.g., vector fields, differential forms... (resp., the Lie derivative, the de Rham differential...) are sheaves (resp., sheaf morphisms). Despite this sheaf-theoretic nature, most textbooks present Differential Geometry in terms of global sections and morphisms between them. Since these sections are sections of vector bundles (resp., these global morphisms are local operators), restriction and gluing is canonical (resp., the existence of smooth bump functions allows us to localize the global morphisms in such a way that they commute with restrictions; e.g., for the de Rham differential, we have

$$
\left.\left(\left.d\right|_{U} \omega_{U}\right)\right|_{V}=\left.\left(d\left(\alpha_{V} \omega_{U}\right)\right)\right|_{V} \quad \text { and }\left.\left.\quad d\right|_{U} \omega\right|_{U}=\left.(d \omega)\right|_{U}
$$

where $\alpha_{V}$ is a bump function with constant value 1 in $V \subset U$ and support in $U$ ). Such global viewpoints are not possible in the real-analytic and holomorphic settings, since no interesting analytic bump functions exist.

There are a number of well-known results on the equivalence of categories of sheaves and the corresponding categories of global sections, essentially when the topological space underlying the considered sheaves is an affine scheme or variety. In the present paper, we use the fact that, for an affine scheme $\left(X, \mathcal{O}_{X}\right)$, there is an equivalence [Har97]

$$
\Gamma(X, \bullet): \operatorname{qcMod}\left(\mathcal{O}_{X}\right) \rightleftarrows \operatorname{Mod}\left(\mathcal{O}_{X}(X)\right): \bullet
$$

between the category of quasi-coherent $\mathcal{O}_{X}$-modules and the category of $\mathcal{O}_{X}(X)$-modules. The functor $\bullet$ is isomorphic to the functor $\mathcal{O}_{X} \otimes_{\mathcal{O}_{X}(X)} \bullet$

\subsection{Appendix 4 - Model categories}

Quite a few non-equivalent definitions of model categories and cofibrantly generated model categories can be found in the literature. In this paper, we use the definitions of [Hov07] and of GS06.

In the definition of model categories, both texts [Hov07] and [GS06] assume the existence of all small limits and colimits in the underlying category - in contrast with Quillen's original definition, which asks only for the existence of finite limits and colimits. However, the 
two references use different 'cofibration - trivial fibration' and 'trivial cofibration - fibration' factorization axioms MC5. Indeed, in GS06, the authors use Quillen's original axiom, which merely requires the existence of the two factorizations, whereas in [Hov07], the author requires the factorizations to be functorial, and even includes the choice of a pair of such functorial factorizations in the axioms of the model structure. However, this difference does not play any role in the present paper, since we are dealing with cofibrantly generated model categories, so that a choice of functorial factorizations is always possible via the small object argument.

For the definitions of cofibrantly generated model structures, some preparation is needed.

An ordinal $\lambda$ is filtered with respect to a cardinal $\kappa$, if $\lambda$ is a limit ordinal such that the supremum of a subset of $\lambda$ of cardinality at most $\kappa$ is smaller than $\lambda$. This condition is actually a largeness condition for $\lambda$ with respect to $\kappa$ : if $\lambda$ is $\kappa$-filtered for $\kappa>\kappa^{\prime}$, then $\lambda$ is also $\kappa^{\prime}$-filtered. For a finite cardinal $\kappa$, a $\kappa$-filtered ordinal is just a limit ordinal.

Smallness of an object $A$ in a category $\mathrm{C}$ (assumed to have all small colimits) is defined with respect to a class of morphisms $W$ in $\mathrm{C}$ and a cardinal $\kappa$ (that can depend on $A$ ) [Hov07]. The point is that the covariant Hom-functor

$$
\mathrm{C}(A, \bullet):=\operatorname{Hom}_{\mathrm{C}}(A, \bullet)
$$

commutes with limits, but usually not with colimits. However, if the considered sequence is sufficiently large with respect to $A$, then commutation may be proven. More precisely, for $A \in \mathrm{C}$, we consider the colimits of all the $\lambda$-sequences (with arrows in $W$ ) for all $\kappa$-filtered ordinals $\lambda$ (usually for $\kappa=\kappa(A)$ ), and try to prove that the covariant Hom-functor $\mathrm{C}(A, \bullet)$ commutes with these colimits. In this case, we say that $A \in \mathrm{C}$ is small with respect to $\kappa$ and $W$. Of course, if $\kappa<\kappa^{\prime}$, then $\kappa$-smallness implies $\kappa^{\prime}$-smallness.

In GS06], 'small' (with respect to $W$ ) means 'sequentially small': the covariant Homfunctor commutes with the colimits of the $\omega$-sequences. This concept matches the notion ' $n$-small', i.e., small relative to a finite cardinal $n \in \mathbb{N}$ : the covariant Hom-functor commutes with the colimits of the $\lambda$-sequences for all limit ordinals $\lambda$. In [Hov07], 'small' (relative to $W$ ) means $\kappa$-small for some $\kappa$ : the covariant Hom-functor commutes with the colimits of all the $\lambda$-sequences for all the $\kappa$-filtered ordinals $\lambda$. It is clear that $n$-small implies $\kappa$-small, for any $\kappa>n$.

More precisely, a $\lambda$-sequence in $\mathrm{C}$ is a colimit respecting functor $X: \lambda \rightarrow \mathrm{C}$. Usually this diagram is denoted by $X_{0} \rightarrow X_{1} \rightarrow \ldots \rightarrow X_{\beta} \rightarrow \ldots$ It is natural to refer to the map

$$
X_{0} \rightarrow \operatorname{colim}_{\beta<\lambda} X_{\beta}
$$

as the composite of the $\lambda$-sequence $X$. If $W$ is a class of morphisms in $\mathrm{C}$ and every map $X_{\beta} \rightarrow X_{\beta+1}, \beta+1<\lambda$, is in $W$, we refer to the composite $X_{0} \rightarrow \operatorname{colim}_{\beta<\lambda} X_{\beta}$ as a transfinite composition of maps in $W$. Let us also recall that, if we have a commutative square in $\mathrm{C}$, the right down arrow is said to be the pushout of the left down arrow. We now denote by $W$-cell the class of transfinite compositions of pushouts of arrows in $W$. It turns out that $W$-cell is a subclass of the class $\operatorname{LLP}(\operatorname{RLP}(W))$ (where notation is self-explaining). 
We are now prepared to give the finite and the transfinite definitions of a cofibrantly generated model category.

A model category is cofibrantly generated [GS06], if there exist sets of morphisms $I$ and $J$, which generate the cofibrations and the trivial cofibrations, respectively, i.e., more precisely, if there are sets $I$ and $J$ such that

1. the source of every morphism in $I$ is sequentially small with respect to the class Cof, and TrivFib $=\operatorname{RLP}(I)$,

2. the source of every morphism in $J$ is sequentially small with respect to the class TrivCof, and $\mathrm{Fib}=\mathrm{RLP}(J)$.

It then follows that $I$ and $J$ are actually the generating cofibrations and the generating trivial cofibrations:

$$
\operatorname{Cof}=\operatorname{LLP}(\operatorname{RLP}(I)) \text { and } \quad \text { TrivCof }=\operatorname{LLP}(\operatorname{RLP}(J)) .
$$

Alternatively, a model category is cofibrantly generated [Hov07], if there exist sets $I$ and $J$ of maps such that

1. the domains of the maps in $I$ are small ( $\kappa$-small for some fixed $\kappa)$ relative to $I$-cell, and TrivFib $=\operatorname{RLP}(I)$,

2. the domains of the maps in $J$ are small ( $\kappa$-small for some fixed $\kappa$ ) relative to $J$-cell, and $\mathrm{Fib}=\operatorname{RLP}(J)$.

It is clear that the finite definition [GS06] is stronger than the transfinite one [Hov07]. First, $n$-smallness implies $\kappa$-smallness, and, second, smallness with respect to Cof (resp., TrivCof) implies smallness with respect to $I$-cell (resp., $J$-cell).

The model structures we study in the present paper are all finitely generated. A finitely generated model structure [Hov07] is a cofibrantly generated model structure, such that $I$ and $J$ can be chosen so that their sources and targets are $n$-small, $n \in \mathbb{N}$, relative to Cof. This implies in particular that our model structures are cofibrantly generated in the sense of GS06.

For more information on model categories, we refer the reader to [GS06], [Hir00], [Hov07, and Qui67]. The background material on category theory can be found in [Bor94a], [Bor94b], and Mac98.

\subsection{Appendix 5 - Invariants versus coinvariants}

If $G$ is a (multiplicative) group and $k$ a commutative unital ring, we denote by $k[G]$ the group $k$-algebra of $G$ (the free $k$-module made of all formal finite linear combinations $\sum_{g \in G} r(g) g$ with coefficients in $k$, endowed with the unital ring multiplication that extends the group multiplication by linearity). 
In the following, we use notation of Subsection 7.1. Observe that $\otimes_{\mathcal{O}}^{n} M_{\bullet}$ is a module over the group $\mathcal{O}$-algebra $\mathcal{O}\left[\mathbb{S}_{n}\right]$, where $\mathbb{S}_{n}$ denotes the $n$-th symmetric group. There is an $\mathcal{O}$-module isomorphism

$$
\mathcal{S}_{\mathcal{O}}^{n} M_{\bullet}=\otimes_{\mathcal{O}}^{n} M_{\bullet} / \mathcal{I} \cap \otimes_{\mathcal{O}}^{n} M_{\bullet} \simeq\left(\otimes_{\mathcal{O}}^{n} M_{\bullet}\right)_{\mathbb{S}_{n}}:=\otimes_{\mathcal{O}}^{n} M_{\bullet} /\langle T-\sigma \cdot T\rangle,
$$

where $\left(\otimes_{\mathcal{O}}^{n} M_{\bullet}\right)_{\mathbb{S}_{n}}$ is the $\mathcal{O}$-module of $\mathbb{S}_{n}$-coinvariants and where the denominator is the $\mathcal{O}$ submodule generated by the elements of the type $T-\sigma \cdot T, T \in \otimes_{\mathcal{O}^{n}}^{n} M_{\bullet}, \sigma \in \mathbb{S}_{n}$ (a Koszul sign is incorporated in the action of $\sigma$ ). It is known that, since the cardinality of $\mathbb{S}_{n}$ is invertible in $\mathcal{O}$, we have also an $\mathcal{O}$-module isomorphism

$$
\left(\otimes_{\mathcal{O}}^{n} M_{\bullet}\right)_{\mathbb{S}_{n}} \simeq\left(\otimes_{\mathcal{O}}^{n} M_{\bullet}\right)^{\mathbb{S}_{n}}:=\left\{T \in \otimes_{\mathcal{O}}^{n} M_{\bullet}: \sigma \cdot T=T, \forall \sigma \in \mathbb{S}_{n}\right\}
$$

between the $\mathbb{S}_{n}$-coinvariants and the $\mathbb{S}_{n}$-invariants. The averaging map or graded symmetrization operator

$$
\mathfrak{S}: \otimes_{\mathcal{O}}^{n} M_{\bullet} \ni T \mapsto \frac{1}{n !} \sum_{\sigma \in \mathbb{S}_{n}} \sigma \cdot T \in\left(\otimes_{\mathcal{O}}^{n} M_{\bullet}\right)^{\mathbb{S}_{n}}
$$

coincides with identity on $\left(\otimes_{\mathcal{O}}^{n} M_{\bullet}\right)^{\mathbb{S}_{n}}$, what implies that it is surjective. When viewed as defined on coinvariants $\left(\otimes_{\mathcal{O}}^{n} M_{\bullet}\right)_{\mathbb{S}_{n}}$, it provides the mentioned isomorphism (93). It is straightforwardly checked that the graded symmetric multiplication $\vee$ on $\left(\otimes_{\mathcal{O}}^{*} M_{\bullet}\right)^{\mathbb{S}_{*}}$, defined by

$$
\mathfrak{S}(S) \vee \mathfrak{S}(T)=\mathfrak{S}(\mathfrak{S}(S) \otimes \mathfrak{S}(T)),
$$

endows $\left(\otimes_{\mathcal{O}}^{*} M_{\bullet}\right)^{\mathbb{S}_{*}}$ with a DG $\mathcal{D}$-algebra structure, and that the $\mathcal{O}$-module isomorphism

$$
\mathcal{S}_{\mathcal{O}}^{*} M_{\bullet} \simeq\left\{T \in \otimes_{\mathcal{O}}^{*} M_{\bullet}: \sigma \cdot T=T, \forall \sigma \in \mathbb{S}_{*}\right\}
$$

is in fact a DGDA-isomorphism.

\subsection{Appendix 6 - Proof of Theorem 29}

The proof of functoriality of the decompositions will be given in Appendix 11.7. Thus, only Part (2) requires immediate explanations. We use again the above-introduced notation $R_{k}=A \otimes \mathcal{S} V_{k}$; we also set $R=A \otimes \mathcal{S} V$. The multiplication in $R_{k}$ (resp., in $R$ ) will be denoted by $\diamond_{k}($ resp., $\diamond)$.

To show that $j$ is a $\mathrm{RSDA}$, we have to check that $A$ is a differential graded $\mathcal{D}$-subalgebra of $R$, that the basis of $V$ is indexed by a well-ordered set and that $d_{2}$ is lowering.

The main idea to keep in mind is that $R=\bigcup_{k} R_{k}$ - so that any element of $R$ belongs to some $R_{k}$ in the increasing sequence $R_{0} \subset R_{1} \subset \ldots$ - and that the DGDA structure on $R$ is defined in the standard manner. For instance, the product of $\mathfrak{a} \otimes X, \mathfrak{b} \otimes Y \in R \cap R_{k}$ is defined by

$$
(\mathfrak{a} \otimes X) \diamond(\mathfrak{b} \otimes Y)=(\mathfrak{a} \otimes X) \diamond_{k}(\mathfrak{b} \otimes Y)=(-1)^{\tilde{X} \tilde{\mathfrak{b}}}(\mathfrak{a} * \mathfrak{b}) \otimes(X \odot Y),
$$

where 'tilde' (resp., *) denotes as usual the degree (resp., the multiplication in $A$ ). It follows that $\diamond$ restricts on $A$ to $*$. Similarly, $\left.d_{2}\right|_{A}=\left.\delta_{0}\right|_{A}=d_{A}$, in view of (71) and (59). Finally, we see that $A$ satisfies actually the mentioned subalgebra condition. 
We now order the basis of $V$. First, we well-order, for any fixed generator degree $m \in \mathbb{N}$ (see (70)), the sets

$$
\left\{s^{-1} \mathbb{I}_{b_{m+1}}\right\},\left\{\mathbb{I}_{b_{m}}\right\},\left\{\mathbb{I}_{\beta_{m}}\right\},\left\{\mathbb{I}_{\sigma_{m-1}, \mathfrak{b}_{m}}^{1}\right\},\left\{\mathbb{I}_{\sigma_{m-1}, \mathfrak{b}_{m}}^{2}\right\}, \ldots
$$

of degree $m$ generators of a given type (for $m=0$, only the sets $\left\{s^{-1} \mathbb{I}_{b_{1}}\right\}$ and $\left\{\mathbb{I}_{\beta_{0}}\right\}$ are nonempty). We totally order the set of all degree $m$ generators by totally ordering its partition (97):

$$
\left\{s^{-1} \mathbb{I}_{b_{m+1}}\right\}<\left\{\mathbb{I}_{b_{m}}\right\}<\left\{\mathbb{I}_{\beta_{m}}\right\}<\left\{\mathbb{I}_{\sigma_{m-1}, \mathfrak{b}_{m}}^{1}\right\}<\left\{\mathbb{I}_{\sigma_{m-1}, \mathfrak{b}_{m}}^{2}\right\}<\ldots
$$

A total order on the set of all generators (of all degrees) is now obtained by declaring that any generator of degree $m$ is smaller than any generator of degree $m+1$. This total order is a well-ordering, since no infinite descending sequence exists in the set of all generators.

Finally, the differential $d_{2}$ sends the first and third types of generators (see (97)) to 0 and it maps the second type to the first. Hence, so far $d_{2}$ is lowering. Further, we have

$$
d_{2}\left(\mathbb{I}_{\sigma_{m-1}, \mathfrak{b}_{m}}^{k}\right)=\sigma_{m-1} \in\left(R_{k-1}\right)_{m-1}
$$

where $m-1$ refers to the term of degree $m-1$ in $R_{k-1}$. Since this term is generated by the generators

$$
\left\{s^{-1} \mathbb{I}_{b_{\ell+1}}\right\},\left\{\mathbb{I}_{b_{\ell}}\right\},\left\{\mathbb{I}_{\beta_{\ell}}\right\},\left\{\mathbb{I}_{\sigma_{\ell-1}, \mathfrak{b}_{\ell}}^{1}\right\}, \ldots,\left\{\mathbb{I}_{\sigma_{\ell-1}, \mathfrak{b}_{\ell}}^{k-1}\right\},
$$

where $\ell<m$, the differential $d_{2}$ is definitely lowering.

It remains to verify that the described construction yields a morphism $q: A \otimes \mathcal{S} V \rightarrow B$ that is actually a trivial fibration.

Since fibrations are exactly the morphisms that are surjective in all positive degrees, and since $q\left|R_{U}=q_{0}\right| R_{U}=p$ is degree-wise surjective, it is clear that $q$ is a fibration. As for triviality, let $\left[\beta_{n}\right] \in H\left(B, d_{B}\right), n \geq 0$. Since $\mathbb{I}_{\beta_{n}} \in \operatorname{ker} \delta_{0} \subset \operatorname{ker} d_{2}$, the homology class $\left[\mathbb{I}_{\beta_{n}}\right] \in H\left(R, d_{2}\right)$ makes sense; moreover,

$$
H(q)\left[\mathbb{I}_{\beta_{n}}\right]=\left[q \mathbb{I}_{\beta_{n}}\right]=\left[q_{0} \mathbb{I}_{\beta_{n}}\right]=\left[\beta_{n}\right],
$$

so that $H(q)$ is surjective. Finally, let $\left[\sigma_{n}\right] \in H\left(R, d_{2}\right)$ and assume that $H(q)\left[\sigma_{n}\right]=0$, i.e., that $q \sigma_{n} \in \operatorname{im} d_{B}$. Since there is a lowest $k \in \mathbb{N}$ such that $\sigma_{n} \in R_{k}$, we have $\sigma_{n} \in \operatorname{ker} \delta_{k}$ and $q_{k} \sigma_{n}=d_{B} \mathfrak{b}_{n+1}$, for some $\mathfrak{b}_{n+1} \in B_{n+1}$. Hence, a pair $\left(\sigma_{n}, \mathfrak{b}_{n+1}\right) \in \mathfrak{B}_{k}$ and a generator $\mathbb{I}_{\sigma_{n}, \mathfrak{b}_{n+1}}^{k+1} \in R_{k+1} \subset R$. Since

$$
\sigma_{n}=\delta_{k+1} \mathbb{I}_{\sigma_{n}, \mathfrak{b}_{n+1}}^{k+1}=d_{2} \mathbb{I}_{\sigma_{n}, \mathfrak{b}_{n+1}}^{k+1},
$$

we obtain that $\left[\sigma_{n}\right]=0$ and that $H(q)$ is injective.

\subsection{Appendix 7 - Explicit functorial cofibrant replacement functor}

(1) We proved in Subsection 7.5 that the factorization $(i, p)=(i(\phi), p(\phi))$ of the DGDAmorphisms $\phi$, described in Theorem 29, is functorial: 
Proposition 33. In DGDA, the functorial fibrant replacement functor $R$, which is induced by the functorial 'TrivCof-Fib' factorization $(i, p)$ of Theorem [29, is the identity functor $R=\mathrm{id}$.

(2) To finish the proof of Theorem 29, we still have to show that the factorization $(j, q)$ is functorial, i.e., that for any commutative DGDA-square

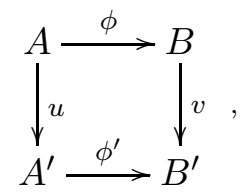

there is a commutative DGDA-diagram

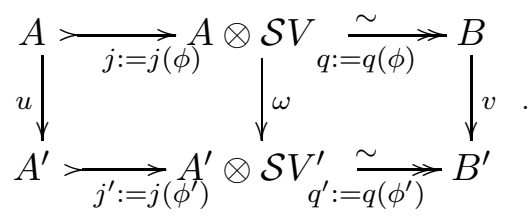

Let us stress that the following proof fails, if we use the non-functorial factorization mentioned in Remark 30 (the critical spots are marked by $\triangleleft$ ).

Just as we constructed in Section 9, the RSDA $R=A \otimes \mathcal{S} V$ (resp., $R^{\prime}=A^{\prime} \otimes \mathcal{S} V^{\prime}$ ) as the colimit of a sequence $R_{k}=A \otimes \mathcal{S} V_{k}$ (resp., $R_{k}^{\prime}=A^{\prime} \otimes \mathcal{S} V_{k}^{\prime}$ ), we will build $\omega \in \operatorname{DGDA}\left(R, R^{\prime}\right)$ as the colimit of a sequence

$$
\omega_{k} \in \operatorname{DGDA}\left(R_{k}, R_{k}^{\prime}\right) .
$$

Recall moreover that $q$ is the colimit of a sequence $q_{k} \in \operatorname{DGDA}\left(R_{k}, B\right)$, and that $j$ is nothing but $j_{k} \in \operatorname{DGDA}\left(A, R_{k}\right)$ viewed as valued in the supalgebra $R$ - and similarly for $q^{\prime}, q_{k}^{\prime}, j^{\prime}, j_{k}^{\prime}$. Since we look for a morphism $\omega$ that makes the left and right squares of the diagram (99) commutative, we will construct $\omega_{k}$ so that

$$
\omega_{k} j_{k}=j_{k}^{\prime} u \text { and } v q_{k}=q_{k}^{\prime} \omega_{k} .
$$

Since the RSDA $A \rightarrow R_{0}=A \otimes \mathcal{S} V_{0}$ is split, we define

$$
\omega_{0} \in \operatorname{DGDA}\left(A \otimes \mathcal{S} V_{0}, R_{0}^{\prime}\right)
$$

as

$$
\omega_{0}=j_{0}^{\prime} u \diamond_{0} w_{0}
$$

where we denoted the multiplication in $R_{0}^{\prime}$ by the same symbol $\diamond_{0}$ as the multiplication in $R_{0}$, where $j_{0}^{\prime} u \in \operatorname{DGDA}\left(A, R_{0}^{\prime}\right)$, and where $w_{0} \in \operatorname{DGDA}\left(\mathcal{S} V_{0}, R_{0}^{\prime}\right)$. As the differential $\delta_{V_{0}}$, see Section 9, has been obtained via Lemma 16, the morphism $w_{0}$ can be built as described in Lemma 17, we set

$$
w_{0}\left(s^{-1} \mathbb{I}_{b_{n}}\right)=s^{-1} \mathbb{I}_{v\left(b_{n}\right)} \in V_{0}^{\prime}, w_{0}\left(\mathbb{I}_{b_{n}}\right)=\mathbb{I}_{v\left(b_{n}\right)} \in V_{0}^{\prime}, \text { and } w_{0}\left(\mathbb{I}_{\beta_{n}}\right)=\mathbb{I}_{v\left(\beta_{n}\right)} \in V_{0}^{\prime},
$$


and easily check that $w_{0} \delta_{V_{0}}=\delta_{0}^{\prime} w_{0}$ on the generators. The first commutation condition (101) is obviously satisfied. As for the verification of the second condition, let $t=\mathfrak{a} \otimes x_{1} \odot \ldots \odot x_{\ell} \in$ $A \otimes \mathcal{S} V_{0}$ and remember (see (61)) that $q_{0}=\phi \star q_{V_{0}}$ and $q_{0}^{\prime}=\phi^{\prime} \star q_{V_{0}^{\prime}}$, where we denoted again the multiplications in $B$ and $B^{\prime}$ by the same symbol $\star$. Then

$$
v q_{0}(t)=v \phi(\mathfrak{a}) \star v q_{V_{0}}\left(x_{1}\right) \star \ldots \star v q_{V_{0}}\left(x_{\ell}\right)
$$

and

$$
q_{0}^{\prime} \omega_{0}(t)=q_{0}^{\prime} j_{0}^{\prime} u(\mathfrak{a}) \star q_{0}^{\prime} w_{0}\left(x_{1}\right) \star \ldots \star q_{0}^{\prime} w_{0}\left(x_{\ell}\right)=\phi^{\prime} u(\mathfrak{a}) \star q_{0}^{\prime} w_{0}\left(x_{1}\right) \star \ldots \star q_{0}^{\prime} w_{0}\left(x_{\ell}\right) .
$$

It thus suffices to show that $v q_{V_{0}}=q_{0}^{\prime} w_{0}$ on the generators $s^{-1} \mathbb{I}_{b_{n}}, \mathbb{I}_{b_{n}}, \mathbb{I}_{\beta_{n}}$ of $V_{0}$, what follows from Equations (60) and (103) $\left(\triangleleft_{1}\right)$.

Assume now that the $\omega_{\ell}$ have been constructed according to the requirements (100) and (101), for all $\ell \in\{0, \ldots, k-1\}$, and build their extension

$$
\omega_{k} \in \operatorname{DGDA}\left(R_{k}, R_{k}^{\prime}\right)
$$

as follows. Since $\omega_{k-1}$, viewed as valued in $R_{k}^{\prime}$, is a morphism $\omega_{k-1} \in \operatorname{DGDA}\left(R_{k-1}, R_{k}^{\prime}\right)$ and since the differential $\delta_{k}$ of $R_{k} \simeq R_{k-1} \otimes \mathcal{S} G_{k}$, where $G_{k}$ is the free $\mathcal{D}$-module

$$
G_{k}=\left\langle\mathbb{I}_{\sigma_{n}, \mathfrak{b}_{n+1}}^{k}:\left(\sigma_{n}, \mathfrak{b}_{n+1}\right) \in \mathfrak{B}_{k-1}\right\rangle,
$$

has been defined by means of Lemma 23. the morphism $\omega_{k}$ is, in view of the same lemma, completely defined by degree $n+1$ values

$$
\omega_{k}\left(\mathbb{I}_{\sigma_{n}, \mathfrak{b}_{n+1}}^{k}\right) \in \delta_{k}^{-1}\left(\omega_{k-1} \delta_{k}\left(\mathbb{I}_{\sigma_{n}, \mathfrak{b}_{n+1}}^{k}\right)\right) .
$$

As the last condition reads

$$
\delta_{k}^{\prime} \omega_{k}\left(\mathbb{I}_{\sigma_{n}, \mathfrak{b}_{n+1}}^{k}\right)=\omega_{k-1}\left(\sigma_{n}\right)
$$

it is natural to set

$$
\omega_{k}\left(\mathbb{I}_{\sigma_{n}, \mathfrak{b}_{n+1}}^{k}\right)=\mathbb{I}_{\omega_{k-1}\left(\sigma_{n}\right), v\left(\mathfrak{b}_{n+1}\right)}^{k},
$$

provided we have

$$
\left(\omega_{k-1}\left(\sigma_{n}\right), v\left(\mathfrak{b}_{n+1}\right)\right) \in \mathfrak{B}_{k-1}^{\prime} \quad\left(\triangleleft_{2}\right) .
$$

This requirement means that $\delta_{k-1}^{\prime} \omega_{k-1}\left(\sigma_{n}\right)=0$ and that $q_{k-1}^{\prime} \omega_{k-1}\left(\sigma_{n}\right)=d_{B^{\prime}} v\left(\mathfrak{b}_{n+1}\right)$. To see that both conditions hold, it suffices to remember that $\left(\sigma_{n}, \mathfrak{b}_{n+1}\right) \in \mathfrak{B}_{k-1}$, that $\omega_{k-1}$ commutes with the differentials, and that it satisfies the second equation (101). Hence the searched morphism $\omega_{k} \in \operatorname{DGDA}\left(R_{k}, R_{k}^{\prime}\right)$, such that $\left.\omega_{k}\right|_{R_{k-1}}=\omega_{k-1}$ (where the RHS is viewed as valued in $R_{k}^{\prime}$ ). To finish the construction of $\omega_{k}$, we must still verify that $\omega_{k}$ complies with (101). The first commutation relation is clearly satisfied. For the second, we consider

$$
r_{k}=r_{k-1} \otimes g_{1} \odot \ldots \odot g_{\ell} \in R_{k-1} \otimes \mathcal{S} G_{k}
$$

and proceed as above: recalling that $\omega_{k}$ and $q_{k}$ have been defined via Equation (34) in Lemma 23. that $q_{k}^{\prime}$ and $v$ are algebra morphisms, and that $\omega_{k-1}$ satisfies (101), we see that it suffices 
to check that $q_{k}^{\prime} \omega_{k}=v q_{k}$ on the generators $\mathbb{I}_{\sigma_{n}, \mathfrak{b}_{n+1}}^{k}$ - what follows immediately from the definitions $\left(\triangleleft_{3}\right)$.

Remember now that $\left(\left(R, d_{2}\right), i_{r}\right)$ is the direct limit of the direct system $\left(\left(R_{k}, \delta_{k}\right), \iota_{s r}\right)$, i.e., that

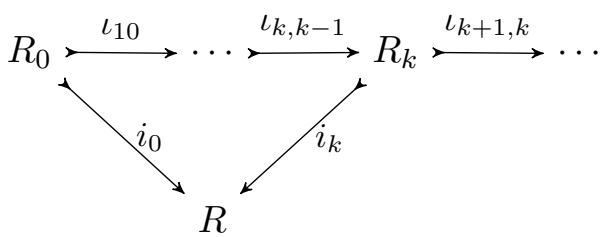

where all arrows are canonical inclusions, and that the same holds for $\left(\left(R^{\prime}, d_{2}^{\prime}\right), i_{r}^{\prime}\right)$ and $\left(\left(R_{k}^{\prime}, \delta_{k}^{\prime}\right), \iota_{s r}^{\prime}\right)$. Since the just defined morphisms $\omega_{k}$ provide morphisms $i_{k}^{\prime} \omega_{k} \in \operatorname{DGDA}\left(R_{k}, R^{\prime}\right)$ (such that the required commutations hold - as $\left.\omega_{k}\right|_{R_{0}}=\omega_{0}$ ), it follows from universality that there is a unique morphism $\omega \in \operatorname{DGDA}\left(R, R^{\prime}\right)$, such that $\omega i_{k}=i_{k}^{\prime} \omega_{k}$, i.e., such that

$$
\left.\omega\right|_{R_{k}}=\omega_{k} .
$$

When using the last result, one easily concludes that $\omega j=j^{\prime} u$ and $v q=q^{\prime} \omega$.

This completes the proof of Theorem 29.

Remark 34. The preceding proof of functoriality fails for the factorization of Remark 30. The latter adds only one new generator $\mathbb{I}_{\dot{\beta}_{n}}$ for each homology class $\dot{\beta}_{n} \simeq\left[\beta_{n}\right]$, and it adds only one new generator $\mathbb{I}_{\sigma_{n}}^{k}$ for each $\sigma_{n} \in \mathcal{B}_{k-1} \backslash \mathcal{B}_{k-2}$, where

$$
\mathcal{B}_{r}=\left\{\sigma_{n} \in \operatorname{ker} \delta_{r}: q_{r} \sigma_{n} \in \operatorname{im} d_{B}, n \geq 0\right\} .
$$

In $\left(\triangleleft_{1}\right)$, we then get that $v q_{V_{0}}\left(\mathbb{I}_{\dot{\beta}_{n}}\right)$ and $q_{0}^{\prime} w_{0}\left(\mathbb{I}_{\dot{\beta}_{n}}\right)$ are homologous, but not necessarily equal. In $\left(\triangleleft_{2}\right)$, although $\sigma_{n} \in \mathcal{B}_{k-1} \backslash \mathcal{B}_{k-2}$, its image $\omega_{k-1}\left(\sigma_{n}\right) \in \mathcal{B}_{k-1}^{\prime}$ may also belong to $\mathcal{B}_{k-2}^{\prime}$. Finally, in $\left(\triangleleft_{3}\right)$, we find that $v q_{k}\left(\mathbb{I}_{\sigma_{n}}^{k}\right)$ and $q_{k}^{\prime} \omega_{k}\left(\mathbb{I}_{\sigma_{n}}^{k}\right)$ differ by a cycle, but do not necessarily coincide.

The next result describes cofibrant replacements.

Theorem 35. In $\mathrm{DGDA}$, the functorial cofibrant replacement functor $Q$, which is induced by the functorial 'Cof - TrivFib' factorization $(j, q)$ described in Theorem [29, is defined on objects $B \in \mathrm{DGDA}$ by $Q(B)=\mathcal{S} V_{B}$, see Theorem 29 and set $A=\mathcal{O}$, and on morphisms $v \in \operatorname{DGDA}\left(B, B^{\prime}\right)$ by $Q(v)=\omega$, see Equations (106), (104), and (103), and set $\omega_{0}=w_{0}$. Moreover, the differential graded $\mathcal{D}$-algebra $\mathcal{S V}_{B}$, see Proposition 30 and set $A=\mathcal{O}$, is a cofibrant replacement of $B$.

\section{Acknowledgments}

The authors are grateful to Jim Stasheff for having read the first version of the present paper. His comments and suggestions allowed to significantly improve their text. 


\section{References}

[Bar10] G. Barnich, Global and gauge symmetries in classical field theories, Series of lectures, Seminar 'Algebraic Topology, Geometry and Physics', University of Luxembourg, homepages.ulb.ac.be/ gbarnich.

[BD04] A. Beilinson and V. Drinfeld, Chiral algebras, American Mathematical Society Colloquium Publications, 51, Amer. Math. Soc., Providence, RI, 2004.

[Bor94a] F. Borceux, Handbook of categorical algebra 1, volume 50 of Encyclopedia of Mathematics and its applications. Cambridge University Press, Cambridge, 1994.

[Bor94b] F. Borceux, Handbook of categorical algebra 2, volume 51 of Encyclopedia of Mathematics and its applications. Cambridge University Press, Cambridge, 1994.

[BPP17] G. di Brino, D. Pištalo, and N. Poncin, Homotopical algebraic context over differential operators, arXiv:1706.05922.

[Cos11] K. Costello, Renormalization and Effective Field Theory, Mathematical Surveys and Monographs Volume, 170, Amer. Math. Soc., Providence, RI, 2011.

[DS96] W. G. Dwyer, J. Spalinski, Homotopy theories and model categories, Springer, 1996.

[FHT01] Y. Félix, S. Halperin, J.-C. Thomas, Rational Homotopy Theory, Graduate Texts in Mathematics, 205, Springer, 2001.

[GM96] S. I. Gelfand, Y. I. Manin Methods of Homological Algebra, Springer, 1996.

[Gil06] J. Gillespie, The flat model structure on complexes of sheaves, Trans. Amer. Math. Soc., 358(7), 2006, 2855-2874.

[GS06] P. Goerss, K. Schemmerhorn, Model Categories and Simplicial Methods, in: Interactions between homotopy theory and algebra, Amer. Math. Soc., Providence, RI, Contemp. Math., 436, 2007, 3-49.

[Gro61] A. Grothendieck, Éléments de géométrie algébrique: II. Étude globale élémentaire de quelques classes de morphismes, Publ. Math. IHÉS, 8, 1961, 5-222.

[Har97] R. Hartshorne, Algebraic Geometry, Graduate Texts in Mathematics 52, Springer, 1997.

[HT92] M. Henneaux and C. Teitelboim, Quantization of Gauge Systems, Princeton University Press, 1992.

[Hes00] K. Hess, A history of rational homotopy theory, in: History of topology, NorthHolland, Amsterdam, 1999, 757-796.

[Hir00] P. Hirschhorn, Model Categories and Their Localizations, Mathematical Surveys and Monographs 99, Amer. Math. Soc., Providence, RI, 2000. 
[Hir05] P. Hirschhorn, Overcategories and undercategories of model categories, http://www-math.mit.edu/ psh/undercat.pdf, 2005.

[HTT08] R. Hotta, K. Takeuchi, and T. Tanisaki, D-Modules, Perverse Sheaves, and Representation Theory, Progress in Mathematics, 236, Birkhäuser, 2008.

[Hov07] M. Hovey, Model Categories, Amer. Math. Soc., Providence, RI, 2007.

[KS90] M. Kashiwara, P. Schapira, Sheaves on Manifolds, Springer Science and Business Media, 1990.

[KRO07] L. H. Kauffman, D. E. Radford, F. J. Oliveira Souza, Hopf Algebras and Generalizations, Contemp. Math., 441, 2007.

[Mac98] S. Mac Lane, Categories for the working mathematician, volume $\mathbf{5}$ of Graduate Texts in Mathematics. Springer-Verlag, New York, second edition, 1998.

[Mum99] D. Mumford, The Red Book of Varieties and Schemes, Lecture Notes in Mathematics 1358, Springer, 1999.

[Nes03] J. Nestruev, Smooth manifolds and observables, Graduate texts in mathematics 220, Springer, 2003.

[Pau11] F. Paugam, Histories and observables in covariant field theory, J. Geo. Phys., 61 (9), 2011, 1675-1702.

[PP17a] D. Pištalo, N. Poncin, On Koszul-Tate resolutions and Sullivan models, arXiv:1708.05936, to appear in Dissertationes Math.

[PP17b] D. Pištalo, N. Poncin, Homotopical Algebraic Geometry over Differential Operators and Applications, to appear in ArXiv, 2017.

[Qui67] D. Quillen, Homotopical algebra, Lecture Notes in Mathematics, 43, SpringerVerlag, Berlin, 1967.

[Sch12] P. Schapira, D-modules, lecture notes, http://www.math.jussieu.fr/ schapira/lectnotes/Dmod.pdf.

[Sch94] J.-P. Schneiders An introduction to D-modules, Algebraic Analysis Meeting (Liège, 1993), Bull. Soc. Roy. Sci. Liège, 63(3-4), 1994, 223-295.

[Ser55] J.-P. Serre, Faisceaux Algébriques Cohérents, Ann. Math., 2nd Ser., 61 (2), 1955, 197-278.

[Swa62] R. G. Swan, Vector Bundles and Projective Modules, Trans. Amer. Math. Soc., 105 (2), 1962, 264-277.

[Tat57] J. Tate, Homology of Noetherian rings and local rings, Illinois J. Math., 1(1), 1957, $14-27$. 
[TV04] B. Toën, G. Vezzosi, From HAG to DAG: derived moduli stacks, Axiomatic, enriched and motivic homotopy theory, 173-216, NATO Sci. Ser. II, Math. Phys. Chem., 131, Kluwer Acad. Publ., Dordrecht, 2004.

[TV08] B. Toën, G. Vezzosi, Homotopical Algebraic Geometry II: geometric stacks and applications, Mem. Amer. Math. Soc., 193 (902), 2008.

[Ver02] A. Verbovetsky, Remarks on two approaches to the horizontal cohomology: compatibility complex and the Koszul-Tate resolution, Acta Appl. Math. 72 (1), 2002, 123-131.

[Vin01] A. Vinogradov, Cohomological Analysis of Partial Differential Equations and Secondary Calculus, Trans. Math. Mono., 204, Amer. Math. Soc., Providence, RI, 2001.

[Wed16] T. Wedhorn, Manifolds, sheaves, and cohomology, Springer Studium Mathematik Master, 2016.

[Wei93] C. A. Weibel, An introduction to homological algebra, Cambridge studies in advanced mathematics, 38, Cambridge University Press, 1993. 